volume 3

ISSUE 3

2015 Settembre

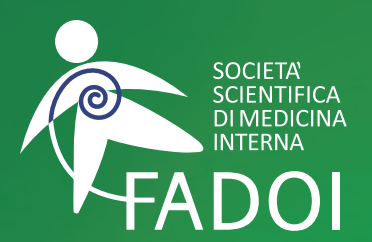

FEDERAZIONE

DELLE ASSOCIAZIONI

DEI DIRIGENTI

OSPEDALIER

INTERNISTI

Editor in Chief Roberto Nardi

The official journal of the Federation of Associations of Hospital Doctors on Internal Medicine (FADOI)

\title{
Il ruolo degli omega-3 nel paziente pluripatologico complesso: dalle evidenze alla pratica clinica in Medicina Interna
}

Guest Editors: P. Gnerre, M. Campanini, A. Fontanella, R. Nardi 


\section{PRESIDENTE ELETTO}

Andrea Fontanella, Napoli, Italy

\section{PAST PRESIDENT}

Carlo Nozzoli, Firenze, Italy

\section{SEGRETARIO}

Giuseppe Augello, Canicattì (AG), Italy

\section{TESORIERE}

Dario Manfellotto, Roma, Italy

DIRETTORE RIVISTA

ITALIAN JOURNAL OF MEDICINE

Roberto Nardi, Bologna, Italy

\section{RESPONSABILE RAPPORTI SOCIETÀ SCIENTIFICHE} E ISTITUZIONI

Antonino Mazzone, Legnano (MI), Italy

\section{RESPONSABILE SISTEMA GESTIONE QUALITÀ}

Franco Berti, Roma, Italy

\section{RESPONSABILE SITO NAZIONALE}

Francesco Cipollini, Ascoli Piceno, Italy

DELEGATO FADOI ITALIAN STROKE ORGANIZATION

Michele Stornello, Siracusa, Italy

\section{COMMISSIONE FADOI GIOVANI}

Paola Gnerre, Savona, Italy

\section{COORDINATORE}

Domenico Panuccio, Bologna, Italy

\section{DIRETTORE SCIENTIFICO}

Gualberto Gussoni, Milano, Italy

DIRETTORE DIPARTIMENTO PER LA FORMAZIONE ED AGGIORNAMENTO

Mauro Silingardi, Guastalla (RE), Italy
DIRETTORE DIPARTIMENTO PER LA RICERCA CLINICA

Giancarlo Agnelli, Perugia, Italy

\section{CONSIGLIO DI AMMINISTRAZIONE}

Consigliere

Consigliere

Consigliere

Consigliere

Consigliere

Consigliere

Consigliere

Consigliere

Consigliere
Paolo Arullani, Roma, Italy Davide Croce, Castellanza (VA), Italy

Francesco D'Amore, Roma, Italy Salvatore Di Rosa, Palermo, Italy

Sandro Fontana, Biella, Italy Ranieri Guerra, Roma, Italy Ido Iori, Reggio Emilia, Italy Giovanni Mathieu, Pinerolo (TO), Italy Cecilia Politi, Isernia, Italy 


\section{QUADERNI - Italian Journal of Medicine}

INTRODUZIONE

II ruolo degli omega-3 nel paziente pluripatologico complesso ....

\section{RASSEGNE}

II ruolo degli omega-3 nella prevenzione dell'ipertrigliceridemia pura e iperlipemia combinata, ma non solo:

dalle evidenze alla pratica clinica

P. Gnerre, O. Para, G. Balbi

Gli omega-3 nell'obesità e nell'insulino-resistenza . . . . . . . . 260 M. Poggiano

Gli omega-3 nel diabete mellito di tipo $2 \ldots \ldots \ldots 262$ M. Poggiano

Gli effetti degli omega-3 sulle aritmie. . . . . . . . . . . . 265 C. Cenci

Gli effetti degli omega-3 nella prevenzione dell'infarto miocardico 269 R. Gerloni

Omega-3 e scompenso cardiaco . . . . . . . . . . . . . . . . . . . . 274 R. Gerloni

I prodotti naturali: una possibile alternativa alle statine per la riduzione del colesterolo . . . . . . . . . . . . . . . . . . . 279 M.C. Pasquini

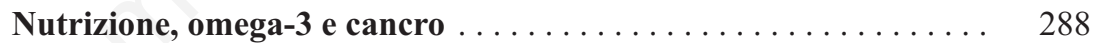
A. Mazza, G. Rubello, G. Mazza

Gli omega-3: trigliceridi naturali o esteri etilici? . . . . . . . . . 301 P. Zuccheri, C. Iacono, G. Benini

II ruolo degli omega-3 nel paziente pluripatologico complesso: dalle evidenze alla pratica clinica in Medicina Interna 306 A. Fontanella 
Farmaci nella forma migliore

\title{
II ruolo degli omega-3 nel paziente pluripatologico complesso
}

\author{
Mauro Campanini, ${ }^{1}$ Roberto Nardi ${ }^{2}$ \\ ${ }^{1}$ Medicina Interna 2 - Dipartimento Medico, AOU Maggiore della Carità, Novara; ${ }^{2}$ Medicina Interna, Bologna, Italia
}

\section{Presentazione}

Gli acidi grassi sono i componenti comuni fondamentali dei lipidi. I più diffusi in natura sono costituiti da un gruppo carbossilico $(-\mathrm{COOH})$, preceduto da una catena di atomi di carbonio $\mathrm{C}$ lineare legata ad atomi di idrogeno $\mathrm{H}$. Gli acidi grassi si differenziano secondo due criteri: in base al numero di atomi di carbonio e a seconda del tipo di legame.

\section{Acidi grassi in base al numero di atomi di carbonio}

Si distinguono: i) acidi grassi a corta catena fino a 4 atomi di carbonio di cui il principale è l'acido butirrico (4:0) il cui ruolo trofico e protettivo a livello della mucosa del colon è di rilevante importanza; ii) acidi grassi a catena media fino a 12 atomi di carbonio con funzione metabolica ed energetica (acido caproico 6:0, acido caprilico 8:0, acido caprico 10:0, acido laurico 12:0); iii) acidi grassi a lunga catena (LCFA) fino a 18 atomi di carbonio con un ruolo energetico, metabolico e strutturale a loro volta classificabili in: a) LCFA saturi (acido miristico 14:0, acido palmitico 16:0, acido stearico 18:0) di cui fanno parte sia i trigliceridi di deposito sia i componenti strutturali di membrana; b) LCFA monoinsaturi (acido oleico 18:1 omega-9) con funzione ipocolesterolemizzante; c) LCFA polinsaturi che sono essenziali (acido linoleico 18:2 omega-6 e acido linolenico 18:3 omega-3; iv) acidi grassi a catena molto lunga

Corrispondente: Roberto Nardi, via C. Pavese 16/2, 40141 Bologna, Italia.

Tel.: +39.335.8291342.

E-mail: nardidoc48@gmail.com

Ringraziamenti: Si ringraziano la dottoressa Luisa Zoni e la dietista Laura Morisi della U.O. Dietologia e Nutrizione Clinica - AUSL di Bologna, Ospedale Maggiore, per la revisione della presentazione.

Articolo pubblicato secondo la Creative Commons Attribution NonCommercial 3.0 License (CC BY-NC 3.0).

(C) Copyright M. Campanini e R. Nardi, 2015

Licensee PAGEPress, Italy

QUADERNI - Italian Journal of Medicine 2015; 3:241-246 da 20 atomi di carbonio in poi (omega-3, omega-6, omega-9) e loro derivati prostaglandine, trombossani e leucotrieni ${ }^{1}$ [L'acido oleico è il più importante acido grasso dell'olio d'oliva: avendo un solo doppio legame è più stabile alle alte temperature, anche se spesso per il suo sapore marcato è meno usato per friggere rispetto a oli ottenuti da semi come soia, girasole o mais che avendo più doppi legami sono polinsaturi. I doppi legami rendono la struttura di questi tipi di olio molto instabile, per questo motivo sarebbe importante non utilizzarli per friggere perché ad alte temperature gli acidi grassi insaturi si ossidano o si denaturano producendo pericolosi radicali liberi e possono dare origine a forme -trans.].

\section{Acidi grassi in base al tipo di legame}

In funzione del tipo di legame gli acidi grassi sono distinti in saturi quando presentano tutti legami semplici (-C-C-) senza doppi legami e insaturi, distinti in monoinsaturi quando hanno un doppio legame $(-\mathrm{C}=\mathrm{C}-) \mathrm{e}$ polinsaturi quando hanno più doppi legami.

\section{Acidi grassi trans}

Gli acidi grassi trans si formano tramite un processo di idrogenazione che consiste nell'aggiunta di atomi di idrogeno sui doppi legami di carbonio. Gli acidi grassi trans sono frequentemente presenti nei prodotti alimentari industriali quali margarine, alimenti fritti, dolciumi, alimenti preconfezionati, ecc. L'industria alimentare si serve di questo processo con lo scopo di rendere solidi gli oli vegetali, che altrimenti a temperatura ambiente sarebbero liquidi. Durante il processo di idrogenazione si formano isomeri -trans degli acidi grassi insaturi (il doppio legame tra i due atomi di carbonio diventa in forma-trans invece che -cis). Alcuni di questi isomeri sono responsabili dell'aumento del rischio di malattie cardiovascolari, dell'aumento delle lipoproteine a bassa densità (LDL) e di una maggiore incidenza di alcune forme di cancro. Alcune forme trans degli acidi grassi insaturi, come gli acidi linoleici coniugati, sono invece presenti naturalmente in alcuni alimenti naturali per un processo di bioidrogenazione che avviene nello stomaco dei ruminanti da parte dei 
batteri intestinali. Recenti studi suggeriscono un ruolo protettivo da malattie cardiache e cancro. Sebbene le ricerche appaiano promettenti, non si può trarre alcuna conclusione certa riguardo il contenuto ottimale, per questo non esistono indicazioni circa la loro assunzione. Si trovano negli alimenti grassi derivanti da animali ruminanti, come il latte intero, i prodotti lattiero caseari e le carni grasse.

I differenti tipi di grassi alimentari con il corrispettivo contenuto di acidi grassi saturi-mono/polinsaturi sono riportati nella Tabella 1.

\section{Acidi grassi essenziali o EFA (essential fatty acid)}

L'organismo umano può sintetizzare tutti gli acidi grassi polinsaturi tranne i 2 precursori: l'acido $\alpha$-linolenico o ALA (C18:3, w3) della famiglia degli omega-3 e l'acido linoleico (C18:2 w6), della famiglia degli omega-6 (Tabella 2). Da questi grassi, comunemente indicati come acidi grassi essenziali o EFA (essential fatty acid), l'uomo è in grado di sintetizzare tutti gli altri polinsaturi, tramite enzimi che consentono di allungare la catena carboniosa (elongasi) e di aumentare il numero di doppi legami (desaturasi), ottenendo diverse serie di composti, a seconda della localizzazione del doppio legame: i) omega-3, quando l'ultimo doppio legame è presente sul terzo carbonio a partire dalla fine (ad es., acido linolenico C 18:3), ovvero acido grasso a 18 atomi di C con tre doppi legami, capostipite degli omega3): ii) omega-6, quando l'ultimo doppio legame è presente sul sesto carbonio a partire dalla fine (ad es., acido linoleico C 18:2), ovvero acido grasso a 18 atomi di $\mathrm{C}$ con due omega- 6 doppi legami, capostipite degli omega-6; c) omega-9 quando l'ultimo doppio legame è presente sul nono carbonio a partire dalla fine (ad es., acido oleico C 18:1) (Tabella 3).,3 La loro essenzialità deriva dal fatto che il nostro organismo non è capace di introdurre doppi legami all'inizio della catena a livello della omega-3 o della omega-6 (ma solo fino al C 9-10).

\section{Gli effetti degli acidi grassi polinsaturi}

Le azioni degli acidi grassi polinsaturi si rivolgono a diverse funzioni, schematicamente riportate nella Tabella $4 .^{4-6}$

A differenza degli omega-6, contenuti negli oli vegetali che la popolazione mediterranea assume in quantità abbondanti per abitudini consolidate, gli acidi grassi omega-3 sono scarsamente rappresentati nella nostra dieta quotidiana. L'acido eicosapentaenoico (EPA) e l'acido docosaesaenoico (DHA) si trovano soprattutto nel pesce di acqua fredda (tonno, salmone e pesce azzurro), nei crostacei e in alcuni

Tabella 1. Differenti tipi di grassi alimentari e rispettivo contenuto di acidi grassi saturi-mono/polinsaturi.

\begin{tabular}{ccccc}
\hline Olio di oliva & Olio di semi di girasole & Olio di arachidi & Olio di palma & Burro \\
\hline - Acidi grassi saturi 14\% & - Acidi grassi saturi $8 \%$ & - Acidi grassi saturi 14\% & - Acidi grassi saturi 49\% & - Acidi grassi saturi 54\% \\
(miristico 1\%; & (miristico 2\%; & (miristico 2\%; & (miristico 1\%; & (miristico 12\%; \\
palmitico 11\%; & palmitico 4\%; & palmitico 9\%; & palmitico 44\%; & palmitico 31\%; \\
stearico 2\%) & stearico 2\%) & stearico 3\%) & stearico 4\%) & stearico 11\%) \\
- Acidi grassi & - Acidi grassi & - Acidi grassi & - Acidi grassi & - Acidi grassi \\
monoinsaturi & monoinsaturi & monoinsaturi & monoinsaturi & monoinsaturi \\
(oleico 72\%) & (oleico 25\%) & (oleico 49\%) & (oleico 37\%) & (oleico 3\%) \\
- Acidi grassi & - Acidi grassi & - Acidi grassi & - Acidi grassi & - Acidi grassi \\
polinsaturi & polinsaturi & polinsaturi & polinsaturi & polinsaturi \\
(linoleico 7\%) & (linoleico 60\%) & (linoleico 31\%) & (linoleico 9\%) & (linoleico 31\%) \\
\hline
\end{tabular}

Tabella 2. Nota all'acronimo $A L A$ : acido $\alpha$-lipoico e $\alpha$-linolenico.

Con nomi simili e talvolta anche la stessa sigla - ALA - acido $\alpha$-linolenico e acido $\alpha$-lipoico non vanno confusi. L'acido $\alpha$-linolenico è un acido grasso omega-3 che non può essere prodotto dall'organismo e per questo ritenuto acido grasso essenziale-EFA, che deve essere acquisito dalla dieta. L'acido $\alpha$-lipoico è uno tra i più potenti antiossidanti fisiologici; svolge un'azione antiossidante protettiva sulle membrane cellulari, mitocondriali e su proteine e DNA. Neutralizza l'azione dei radicali liberi, responsabili dei processi di invecchiamento e diminuzione della funzionalità dei tessuti, e promuove la rigenerazione di altri antiossidanti endogeni come vitamina $\mathrm{E}$, vitamina $\mathrm{C}$ e glutatione. Agisce inoltre come coenzima del metabolismo cellulare, di fondamentale importanza per promuovere i cicli metabolici finalizzati alla produzione di ATP e dunque di energia per la cellula. Si trova in abbondanza in patate, broccoli e spinaci, ma la sua fonte prevalente è costituita dalle carni rosse, in particolare fegato e cuore.

EFA, essential fatty acid. 
oli vegetali (olio di semi di lino, olio di nocciole, olio di colza) che tuttavia sono più ricchi in ALA. L'assunzione di EFA è fondamentale per lo stato di salute, dal momento che essi sono importanti costituenti delle membrane cellulari di organi e tessuti e sono in grado di modulare positivamente diversi processi biologici, ai fini di protezione da eventi fisiologici e patologici. La carenza di EFA può comportare conseguenze di vario tipo: ritardo di crescita, problemi riproduttivi, lesioni cutanee, disturbi renali e epatici, alterata sintesi di lipoproteine, problemi neurologici e visivi, malattie croniche intestinali, alterato metabolismo degli acidi grassi, problemi comportamentali, quali difetti dell'attenzione ed iperattività. Il ruolo nella dieta degli omega3 EPA e DHA nella prevenzione è ben consolidato, ${ }^{7-10}$ con effetti reciprocamente sinergici e complementari. ${ }^{11}$ Il DHA svolge una funzione strutturale, in quanto presente nei fosfolipidi dei sinaptosomi cerebrali, nella retina e nei fosfolipidi dei canali intramembrana del sodio e influenza positivamente l'acuità visiva e le funzioni cognitive. ${ }^{12}$ L'EPA è il principale precursore delle prostaglandine della serie
3, le quali posseggono un'importante attività antiaggregante piastrinica. ${ }^{2}$ Non solo l'eccessivo introito calorico, ma anche lo scarso apporto di EPA-DHA ha conseguenze rilevanti, tanto da essere indicato come seconda causa più frequente di decesso negli USA correlato ad errate abitudini dietetiche (Figura 1). ${ }^{13}$ Ciò a conferma del fatto che la dieta mediterranea, con frutta, verdura, cereali integrali, derivati magri del latte, calcio, vitamina $\mathrm{D}$, acidi grassi monoinsaturi e omega-3, costituisce un consolidato strumento di prevenzione cardiovascolare e della sindrome metabolica. ${ }^{14,15} \mathrm{Si}$ consiglia infatti di privilegiare l'utilizzo di grassi di origine vegetale, soprattutto olio extravergine di oliva e olio di semi monoseme, limitando quelli di origine animale e di incrementare l'assunzione settimanale di pesce (fresco o surgelato, almeno 2-3 volte) in particolare: salmone, alici, sarde, sgombro e tonno, ricchi in omega-3. ${ }^{16}$ Per un approfondimento si rimanda al documento di sintesi della Società Italiana di Nutrizione Umana, che nel 2012 ha rivisto i livelli di assunzione di riferimento di nutrienti ed energia per la popolazione italiana. ${ }^{17}$

Gli omega-3 hanno un ruolo fondamentale per la

Tabella 3. Acidi grassi insaturi omega-3, omega-6, omega-7 e omega-9.

\begin{tabular}{|c|c|c|c|}
\hline Omega-3 & Omega-6 & Omega-7 & Omega-9 \\
\hline Polinsaturi & Polinsaturi & Monoinsaturi & Monoinsaturi \\
\hline $\begin{array}{l}\alpha \text {-linolenico (ALA o LNA) } \\
\text { - C18:3 w3 }\end{array}$ & $\begin{array}{l}\text { Linoleico (LA) } \\
\text { - C18:2 w6 }\end{array}$ & $\begin{array}{l}\text { Palmitoleico - 16:1 }(n-7) \\
\text { - acido 9-esadecenoico }\end{array}$ & $\begin{array}{l}\text { Oleico - 18:1 }(n-9)- \\
\text { acido 9-ottadecenoico }\end{array}$ \\
\hline $\begin{array}{l}\text { Eicosapentaenoico (EPA) } \\
- \text { C20:5 w3 }\end{array}$ & $\begin{array}{l}\text { Gammalinolenico (GLA) } \\
\text { - C18:3 w6 }\end{array}$ & $\begin{array}{l}\text { Vaccenico - 18:1 }(n-7) \\
\text { acido 11-ottadecenoico }\end{array}$ & $\begin{array}{l}\text { Eicosenoico - 20:1 }(n-9) \text { - } \\
\text { acido 11-eicosenoico }\end{array}$ \\
\hline $\begin{array}{l}\text { Docosaenoico (DHA) } \\
- \text { C22:6 w3 }\end{array}$ & $\begin{array}{l}\text { Arachidonico (AA) } \\
\text { - C20:4 w6 }\end{array}$ & & $\begin{array}{l}\text { Eicosatrienoico - 20:3 }(n-9)- \\
\text { acido 5,8,11-eicosatrienoico } \\
\text { Erucico- 22:1 (n-9)- } \\
\text { acido } 13 \text {-docosenoico } \\
\text { Nervonico- } 24: 1(n-9) \text { - } \\
\text { acido } 15 \text {-tetracosenoico }\end{array}$ \\
\hline
\end{tabular}

Fonti principali

ALA: olio di semi di lino, olio di soia, chia, noci

DHA: pesce (acciughe, sardine, aringhe, sgombro, salmone, tonno, pesce spada, halibut e krill), molluschi, alghe

(es., wakame e nori)

EPA: pesce (acciughe, sardine, aringhe, sgombro, salmone, tonno, pesce spada, halibut e krill), molluschi, alghe

(es., wakame e nori)
In primis olio di vinacciolo, di germi di grano, di soia Inoltre: olio di girasole, noci, olio di riso, semi di papavero e sesamo
Palmitoleico 16:1 (N7) noci macamia, olio di pesce Inoltre: lardo e grassi animali

Vaccenico 18:1 latte/latticini di animali ruminanti (mucca, capra e pecora)
Olio di oliva, olio di mandorle, olio di arachidi

ALA: olio di semi di lino,

di soja, di noci, di colza

ALA, acido $\alpha$-linolenico; DHA, acido docosaesaenoico; EPA, acido eicosapentaenoico. 
salute dell'organismo, in quanto svolgono importanti funzioni per la crescita, la produzione di energia, l'integrità della membrana cellulare in quanto costituenti dei fosfolipidi e di quella dei mitocondri, come mediatori chimici a livello cellulare, per la sintesi dell'emoglobina, la funzione sessuale e la riproduzione, per alcune patologie della pelle e retiniche, per una miglior tolleranza dei carboidrati nei diabetici; per la riduzione di colesterolo totale e LDL ed altre molteplici funzioni ancora. Le raccomandazioni della FAO a proposito degli acidi poli-insaturi sono riportate nella Tabella $5 .{ }^{18}$

Una diminuzione o assenza di acidi grassi omega3 comporta una maggiore produzione di acido arachidonico, aumento dell'aggregazione piastrinica con formazione di trombossani, aumentata attività macrofagica, maggior formazione di interleuchina 1 , di leucotrieni 4, del platelet activating factor e del platelet derived growth factor, incremento delle

Tabella 4. Le diverse azioni biologiche ipotizzate per i vari tipi di acidi grassi insaturi.

\begin{tabular}{|c|c|c|c|}
\hline Omega-3 & Omega-6 & Omega-7 & Omega-9 \\
\hline $\begin{array}{l}\text { - Attività antiaterogena, } \\
\text { antinfiammatoria, antitrombotica, } \\
\text { antiaritmica, ipolipemizzante, con } \\
\text { proprietà vasodilatatorie ed } \\
\text { antiaggreganti, effetti benefici sul } \\
\text { microcircolo, di miglioramento } \\
\text { della funzione endoteliale e di } \\
\text { riduzione della pressione arteriosa, } \\
\text { azioni antidepressive e sulla } \\
\text { capacità di attenzione, effetti utili } \\
\text { per il sistema immunitario e la } \\
\text { difesa contro i tumori } \\
\text { - } \uparrow \text { formazione di prostaglandine } \\
\text { PGI3; } \\
\text { - } \uparrow \text { produzione di leucotrieni b5 } \\
\text { (molto meno infiammatori rispetto } \\
\text { ai leucotrieni b4) } \\
\text { - } \uparrow \text { produzione di interleuchina } 2 \\
\text { e EDRF (endothelial derived } \\
\text { Relaxing factor) } \\
\text { - } \uparrow \text { attività fibrinolitica } \\
\text { - Aumento delle HDL }\end{array}$ & $\begin{array}{l}\text { - L'acido arachidonico (aa) è } \\
\text { presente nei fosfolipidi di } \\
\text { membrana ed è importante, insieme } \\
\text { al DHA, nello sviluppo embrionale } \\
\text { e nell'accrescimento del bambino } \\
\text { - L'aa produce le prostaglandine } \\
\text { della serie } 2 \text { (attraverso la via } \\
\text { ciclossigenasica), dando luogo alla } \\
\text { formazione di intermedi metabolici } \\
\text { ad attività pro-infiammatoria e } \\
\text { proaggregante piastrinica } \\
\text { (trombossano a2) } \\
\text { - Dall'acido arachidonico tramite } \\
\text { la via lipossige-nasica si formano } \\
\text { i leucotrieni, che hanno un'azione } \\
\text { bronco-costrittrice (importante } \\
\text { nell'anafilassi insieme alle SRSA } \\
\text { (slow reacting substance of } \\
\text { anaphylaxis) }\end{array}$ & $\begin{array}{l}\text { - L'acido palmitoleico aumenta i } \\
\text { livelli di colesterolo LDL in } \\
\text { pazienti ipercolesterolemici, } \\
\text { anche quando l'apporto } \\
\text { alimentare di colesterolo è basso } \\
\text { e riduce l'HDL colesterolo } \\
\text { - Nei prodotti cosmetici ha un } \\
\text { effetto emolliente sulla pelle }\end{array}$ & $\begin{array}{l}\text { - Il principale acido grasso } \\
\text { monoinsaturo della serie omega-9 } \\
\text { è l'acido oleico, il grasso } \\
\text { principale dell'olio di oliva } \\
\text { - L'acido oleico facilita, se non } \\
\text { rettificato, l'assorbimento delle } \\
\text { vitamine liposolubili e del calcio } \\
\text { - Nell'olio extravergine d'oliva i } \\
\text { componenti benefici per la salute } \\
\text { sono sostanzialmente due: } \\
\text { i) polifenoli, composti chimici } \\
\text { fortemente antiossidanti (in grado di } \\
\text { contrastare i radicali liberi) che } \\
\text { favoriscono la riduzione del } \\
\text { colesterolo cattivo (LDL); } \\
\text { ii) gli acidi grassi insaturi, in } \\
\text { particolare l'acido oleico, omega-9 } \\
\text { monoinsaturo, che costituisce il } \\
70 \% \text { dell'olio } \\
\text { - Secondo la FDA due cucchiai al } \\
\text { giorno di olio di oliva sono utili per } \\
\text { ridurre il rischio di danni coronarici } \\
\text { da grassi saturi }\end{array}$ \\
\hline
\end{tabular}

DHA, acido docosaesaenoico; HDL, lipoproteine ad alta densità; LDL, lipoproteine a bassa densità.

\section{FATTORE DI RISCHIO}

\begin{tabular}{|r|c|}
\hline ECCESSO DI SALE & 102.000 \\
SCARSO APPORTO DI EPA-DHA OMEGA-3 & 84.000 \\
\cline { 2 - 2 } ECCESSIVO APPORTO DI ACIDI GRASSI TRANS & 82.000 \\
ABUSO DI ALCOOL & 64.000 \\
BASSO CONSUMO DI VEGETALI E FRUTTA & 58.000 \\
\hline $\begin{array}{c}\text { ECCESSO DI AC. GRASSI SATURI, SCARSO } \\
\text { APPORTO DI PUFA }\end{array}$ & 15.000 \\
\hline
\end{tabular}

Figura 1. Decessi annuali da tutte le cause per fattori correlati alla dieta negli USA. EPA, acido eicosapentaenoico; DHA, acido docosaesaenoico; PUFA, acidi grassi polinsaturi. Modificata da Danaei et al., 2009. ${ }^{13}$ 
Tabella 5. Raccomandazioni FAO a proposito degli acidi poli-insaturi.

\begin{tabular}{|c|c|}
\hline - Il rapporto omega-6/omega-3 negli alimenti dovre & be collocarsi tra $5: 1$ e $10: 1$ \\
\hline - Chi introduce rapporti maggiori, deve compensare & con alimenti ricchi di omega-3 (es., pesci) \\
\hline - Particolare attenzione a tale rapporto deve essere $\mathrm{p}$ & osta per le donne in gravidanza \\
\hline Nel fabbisogno giornaliero* la quota consigliata è: & $\begin{array}{l}\text { i) per un maschio: } 6 \mathrm{~g} / \text { die di omega- } 6 \text { e } 1,5 \text { di omega- } 3 \\
\text { ii) per una femmina: } 5 \mathrm{~g} / \text { die di omega- } 6 \text { e } 1 \text { di omega- } 3\end{array}$ \\
\hline
\end{tabular}

*Il fabbisogno comunque varia in funzione dell'età.

LDL, delle lipoproteine a densità molto bassa, dei trigliceridi e della viscosità ematica. ${ }^{2}$ In conclusione, gli acidi grassi della serie omega- 3 condizionano profondamente diversi tipi di risposte biologiche, influenzando la stabilità e la fluidità di membrana, la mobilità delle cellule, la formazione di recettori ed il legame di ligandi ai loro recettori, l'attivazione di vie di segnalazione intracellulare direttamente o attraverso la formazione di eicosanoidi, l'espressione genica e la differenziazione cellulare, agendo come veri e propri immunonutrienti. ${ }^{19}$ Effetti benefici sono stati descritti per diversi stati patologici, quali broncopneumopatia cronica ostruttiva, asma, artrite reumatoide, psoriasi, morbo di Crohn, depressione, schizofrenia e malattie neurodegenerative. ${ }^{20}$ In sostanza, gli effetti utili attribuibili agli omega-3, similmente alle statine, sembrerebbero dovuti ad una loro potenziale azione pleiotropica, con effetti clinici conseguenti, ${ }^{21}$ che potrebbero risultare particolarmente vantaggiosi nel paziente internistico complesso, in ragione delle diverse comorbilità esistenti. Scopo di questa monografia è proprio quello di un approfondimento sulle diverse tematiche in causa, affidando alla comunità scientifica la discussione sul tema, a fronte delle evidenze disponibili e delle esplicite limitazioni poste dalle normative esistenti nell'impiego degli omega-3 nella prevenzione primaria e secondaria. ${ }^{22,23}$

\section{Bibliografia}

1. Ciaccio M. Gli acidi grassi omega-3 ed omega-6: dalla biochimica all'applicazione clinica; 2002. Disponibile su: http://www.sinu.it/articoli/topics-04-2002.pdf

2. Boscarini M, Corio R. Gli acidi grassi della serie omegae le loro funzioni. Natural 2005;1:66-74.

3. Mukherjee KD, Kiewitt I. Formation of (n-9) and (n-7) cis-monounsaturated fatty acids in seeds of higher plants. Planta 1980;149:461-3.

4. Din JN, Newby DE, Flapan AD. Omega-3 fatty acids and cardiovascular disease-fishing for a natural treatment. BMJ 2004;328:30-5.

5. Wijendran V, Hayes KC. Dietary n-6 and n-3 fatty acid balance and cardiovascular health. Annu Rev Nutr 2004;24:597-615.
6. Nestel P, Clifton P, Noakes M. Effects of increasing dietary palmitoleic acid compared with palmitic and oleic acids on plasma lipids of hypercholesterolemic men. J Lipid Res 1994;35:656-62.

7. Hibbeln JR, Nieminen LR, Blasbalg TL, et al. Healthy intakes of n-3 and n-6 fatty acids: estimations considering worldwide diversity. Am J Clin Nutr 2006;83:1483S-93S.

8. Mozaffarian D. Fish and n-3 fatty acids for the prevention of fatal coronary heart disease and sudden cardiac death. Am J Clin Nutr 2008;87:1991S-6S.

9. Kris-Etherton PM, Innis S. Position of the American Dietetic Association and Dietitians of Canada: dietary fatty acids. J Am Diet Assoc 2007;107:599-611.

10. Balk E, Chung M, Lichtenstein A, et al. Effects of omega-3 fatty acids on cardiovascular risk factors and intermediate markers of cardiovascular disease. Summary, evidence report/technology assessment: number 93. AHRQ Publication Number 04-E010-1, March 2004. Agency for Healthcare Research and Quality, Rockville, MD, USA. Disponibile su: http://www.ahrq. gov/clinic/epcsums/o3cardrisksum.htm

11. Mozaffarian D, Wu JH. (n-3) fatty acids and cardiovascular health: are effects of EPA and DHA shared or complementary? J Nutr 2012;142:614S-625S.

12. Gil A, Gil F. Fish, a Mediterranean source of n-3 PUFA: benefits do not justify limiting consumption. Br J Nutr 2015;113:S58-67.

13. Danaei G, Ding EL, Mozaffarian D, et al. The preventable causes of death in the United States: comparative risk assessment of dietary, lifestyle, and metabolic risk factors. PLoS Med 2009;6:e1000058.

14. GISSI Prevenzione Investigators. n-3 PUFA and vitamin E in 11,324 post-MI patients: Results of the GISSI Prevenzione trial. Lancet 1999;354:447-55.

15. Calton EK, James AP, Pannu PK, Soares MJ. Certain dietary patterns are beneficial for the metabolic syndrome: reviewing the evidence. Nutr Res 2014;34:559-68.

16. Istituto Nazionale di Ricerca per gli Alimenti e la Nutrizione (INRAN). Linee guida per una sana alimentazione italiana; Revisione 2003. Disponibile su: http:// nut.entecra.it/649/Introduzione.html

17. Società Italiana di Nutrizione Umana (SINU). LARN Livelli di assunzione di riferimento di nutrienti ed energia per la popolazione italiana; Revisione 2012 - Documento di sintesi. Disponibile su: http://www.sinu.it/ $\mathrm{html} / \mathrm{pag} /$ nuovi_larn.asp

18. [No authors listed]. Fats and fatty acids in human nutrition. Proceedings of the Joint FAO/WHO Expert Consultation. November 10-14, 2008. Geneva, Switzerland. Ann Nutr Metab 2009;55:5-300. 
19. Alexander JW. Immunonutrition: the role of omega-fatty acids. Nutrition 1998;14:627-33.

20. Marangoni F, Poli A. Omega-3 e salute cardiovascolare. Riv Soc Ital Med Gen 2010;5:57-61.

21. Mozaffarian D, Rimm EB. Fish intake, contaminants, and human health: evaluating the risks and the benefits. JAMA 2006;296:1885-99.

22. CeVEAS (Centro per la Valutazione della Efficacia della
Assistenza Sanitaria). Pacchetto informativo sui farmaci n. 14 - 2005. Disponibile su: http://assr.regione.emiliaromagna.it/it/servizi/pubblicazioni/collane-cessate/archivio-pacchetti/omega-3-.-quale-ruolo-in-terapia

23. AIFA (Agenzia Italiana del Farmaco). Determinazione n. 1081 del 22/11/2013 - Istituzione della nota AIFA n. 94. Disponibile su: http:/www.agenziafarmaco.gov.it/ sites/default/files/nota94_AIFA.pdf 
Farmaci nella forma migliore

\title{
II ruolo degli omega-3 nella prevenzione dell'ipertrigliceridemia pura e iperlipemia combinata, ma non solo: dalle evidenze alla pratica clinica
}

\author{
Paola Gnerre, ${ }^{1}$ Ombretta Para, ${ }^{2}$ Giulia Balbi ${ }^{3}$ \\ ${ }^{1}$ Medicina Interna, Ospedale San Paolo, Savona; ${ }^{2}$ Medicina Interna, Ospedale Careggi, Firenze; ${ }^{3}$ Medicina Generale, Ospedale \\ S. Bortolo, Vicenza, Italia
}

\section{Introduzione}

Nei paesi occidentali le malattie cardiovascolari (CVD) sono tra le principali cause di morbidità e mortalità. Alti livelli plasmatici di lipoproteine a bassa densità (c-LDL), bassi livelli di lipoproteine ad alta densità (c-HDL), l'ipertensione arteriosa, il diabete, l'obesità, la sindrome metabolica e il fumo sono i principali fattori di rischio per CVD. L'associazione tra elevati livelli di colesterolo c-LDL e l'aumentato rischio di malattie cardiovascolari premature fu definitivamente confermato in una serie di trials condotti nel 1984. ${ }^{1}$ Per malattia coronarica prematura si intende la comparsa di infarto miocardico acuto o il ricorso a procedure di rivascolarizzazione coronarica prima dei 55 anni per gli uomini e dei 65 anni per le donne. Questi limiti potrebbero essere elevati di 5-10 anni nei soggetti non fumatori. La stratificazione e il precoce trattamento dei pazienti con dislipidemia diventa pertanto un elemento essenziale di prevenzione cardiovascolare. La nostra trattazione ha pertanto l'obiettivo di fornire un pratico manuale per un corretto management dei pazienti affetti da dislipidemia oltre a fornire delle indicazioni sull'efficacia dell'utilizzo degli omega-3 in determinate condizioni cliniche. ${ }^{2}$

\section{Le dislipidemie: inquadramento laboratoristico}

I lipidi sono sostanze insolubili in acqua e solubili in solventi organici (etere, alcool, ecc.) composti da

Corrispondente: Paola Gnerre, Medicina Interna, Ospedale San Paolo, via Genova 30, 17100 Savona, Italy.

Tel.: +30.019.8404082. E-mail: pgnerre@yahoo.it

Articolo pubblicato secondo la Creative Commons Attribution NonCommercial 3.0 License (CC BY-NC 3.0).

C Copyright P. Gnerre et al., 2015

Licensee PAGEPress, Italy

QUADERNI - Italian Journal of Medicine 2015; 3:247-259 un gruppo estremamente eterogeneo di molecole (acidi grassi liberi, trigliceridi, fosfolipidi, steroidi, vitamine liposolubili, carotenoidi, ecc.).

Le lipoproteine si classificano in base alla migrazione elettroforetica, in $\alpha$-lipoproteine (HDL), pre- $\beta$ lipoproteine (VLDL), $\beta$-lipoproteine (LDL) e chilomicroni ed in base alla densità crescente in chilomicroni in VLDL, lipoproteine a densità intermedia (IDL), LDL e HDL. Secondo la classificazione di Fredrickson $^{3}$ le dislipidemie vengono classificate in cinque fenotipi: il tipo I, l'ipertrigliceridemia o l'iperchilomicronemia, il tipo II, l'iper- $\beta$-lipoproteinemia, il tipo III, l'iperlipidemia familiare combinata, il tipo IV l'iper-pre- $\beta$-lipoproteinemia, e il tipo V l'iperlipidemia mista (combinazione del tipo I e del tipo IV) (Tabella 1). ${ }^{3}$

\section{Ipertrigliceridemia}

I trigliceridi presenti nel plasma sono in parte esogeni (originati dai grassi introdotti con la dieta) trasportati nei chilomicroni ed in parte endogeni (di sintesi epatica) trasportati nelle VLDL. Nel muscolo e nel tessuto adiposo i chilomicroni e le VLDL sono idrolizzate dalla lipasi lipoproteica (LPL) ad acidi grassi liberi. Normalmente i livelli ematici di trigliceridi (TG) sono $<150 \mathrm{mg} / \mathrm{dL}$. Si parla di valori borderline tra $150-199 \mathrm{mg} / \mathrm{dL}$, valori alti tra $200-499 \mathrm{mg} / \mathrm{dL}$ e molto alti $>500 \mathrm{mg} / \mathrm{dL}$. L'ipertrigliceridemia è una comune forma di dislipidemia frequentemente associata a malattia coronarica prematura e con valori $>500 \mathrm{mg} / \mathrm{dL}$ aumenta significativamente anche il rischio di pancreatite acuta. ${ }^{4}$ Se l'ipertrigliceridemia causa malattia coronarica o sia un marker per altre anormalità di lipoproteine che causano malattia coronarica è a tutt'oggi un aspetto ancora controverso. È sicuramente un dato certo la correlazione dell'ipertrigliceridemia con l'incremento del colesterolo LDL e la riduzione del colesterolo HDL entrambi correlati ad un aumentato rischio cardiovascolare. L'ipertrigliceridemia può essere conseguente ad un incrementata produzione di TG o una ridotta eliminazione e tra le cause riconosce forme genetiche e forme secondarie 
(Tabella 2) ${ }^{4}$ Non di rado però le cause genetiche si combinano a quelle secondarie complicando l'eziologia e la diagnosi. L'iperlipidemia familiare combinata e l'ipertrigliceridemia da deficit familiare della lipasi lipoproteica sono presenti nell' $1 \%$ della popolazione. Questi due disordini se concomitano con una patologia diabetica sono responsabili di quasi il 50\% di tutti gli eventi cardiaci prematuri. Tra le cause secondarie si annoverano scorrette abitudini alimentari, la sindrome metabolica, l'ipotiroidismo, l'insufficienza renale cronica, la sindrome nefrosica, la sindrome di Cushing e alcuni farmaci (Tabella 2).

\section{Iperchilomicronemia (primary hyperchylomicronemia)}

L'iperchilomicronemia primitiva è caratterizzata da una marcata ipertrigliceridemia dovuto ad un incremento dei chilomicroni frequentemente responsabili di episodi di pancreatite acuta e xantomi cutanei. Questa entità include la deficienza familiare della LPL, familial lipoprotein lipase deficiency, la deficienza familiare dell'apoliproteina $\mathrm{C}$, familial apolipoprotein C-II deficiency, l'iperlipoproteinemia primitiva tipo $\mathrm{V}$, primary type $V$ hyperlipoproteine-

Tabella 1. Dislipidemie: classificazione secondo Fredrickson.

\begin{tabular}{|c|c|c|c|c|}
\hline Fenotipo & Caratteristiche & Trigliceridi & Colesterolo & Lipoproteine \\
\hline $\begin{array}{l}\text { I } \\
\text { Iperchilomicronemia } \\
\text { o ipertrigliceridemia } \\
\text { esogena }\end{array}$ & $\begin{array}{l}\text { Deficit familiare di lipasi } \\
\text { lipoproteica }\end{array}$ & $\begin{array}{l}\text { Molto } \\
\text { aumentati }\end{array}$ & Normale & $\begin{array}{l}\uparrow \text { Chilomicroni } \\
\downarrow \text { HDL }\end{array}$ \\
\hline $\begin{array}{l}\text { IIa } \\
\text { Ipercolesterolemia } \\
\text { familiare o iper- } \beta \text { - } \\
\text { lipoproteinemia }\end{array}$ & $\begin{array}{l}\text { Difetto dei recettori } \\
\text { per le LDL }\end{array}$ & Normali & $\begin{array}{l}\text { Molto } \\
\text { aumentato }\end{array}$ & $\begin{array}{l}\uparrow \uparrow \text { Iper- } \beta \text {-lipoproteinemia } \\
(\mathrm{LDL})\end{array}$ \\
\hline $\begin{array}{l}\text { IIb } \\
\text { Iperlipidemia familiare } \\
\text { combinata }\end{array}$ & $\begin{array}{l}\text { Aumento della sintesi } \\
\text { di colesterolo e apo B } \\
\text { Difetto recettoriale }\end{array}$ & Aumentati & $\begin{array}{l}\text { Molto } \\
\text { aumentato }\end{array}$ & $\begin{array}{l}\text { Iper- } \beta \text { e iper-pre- } \beta \text {-lipoproteinemia } \\
(\uparrow \text { LDL, VLDL) }\end{array}$ \\
\hline $\begin{array}{l}\text { III } \\
\text { Iper-pre- } \beta \text {-lipoproteinemia } \\
(\beta \text {-lipoproteinemia allargata) }\end{array}$ & $\begin{array}{l}\text { Carenza o inattività di apo E-3 } \\
\text { con riduzione della clearance } \\
\text { dei remnants }\end{array}$ & Aumentati & Aumentato & $\begin{array}{l}\text { Iper-pre- } \beta \text {-lipoproteinemia } \\
\text { e iper- } \beta \text {-lipoproteinemia } \\
(\text { broad } \beta)(\beta \text {-VLDL })\end{array}$ \\
\hline $\begin{array}{l}\text { IV } \\
\text { Iper-pre- } \beta \text {-lipoproteinemia } \\
\text { o ipertrigliceridemia familiare }\end{array}$ & $\begin{array}{l}\text { Aumentata produzione di } \\
\text { VLDL }\end{array}$ & $\begin{array}{l}\text { Molto } \\
\text { aumentati }\end{array}$ & Aumentato & $\begin{array}{l}\text { Iper-pre- } \beta \text {-lipoproteinemia } \\
(\uparrow \text { VLDL) }\end{array}$ \\
\hline $\begin{array}{l}\mathrm{V} \\
\text { Forma mista }\end{array}$ & $\begin{array}{l}\text { Aumentata produzione } \\
\text { di VLDL } \\
\text { Difettosa clearance } \\
\text { dei remnants aumentati }\end{array}$ & $\begin{array}{l}\text { Molto } \\
\text { Aumentato }\end{array}$ & & $\begin{array}{l}\text { Chilomicroni e } \\
\text { iper-pre- } \beta \text {-lipoproteinemia } \\
\text { ( } \uparrow \text { chilomicroni e VLDL) }\end{array}$ \\
\hline
\end{tabular}

HDL, $\alpha$-lipoproteine; LDL, $\beta$-lipoproteine; VLDL, pre- $\beta$-lipoproteine.

Tabella 2. Cause di ipertrigliceridemia.

\begin{tabular}{ll}
\hline Cause genetiche & Cause secondarie \\
\hline Deficit familiare di lipasi lipoproteica (fenotipo I) & Diabete mellito scompensato \\
\hline Deficienza dell'apo C-II & Sindrome di Cushing \\
\hline Variante genetica di APOC3 e APOA5 & $\begin{array}{l}\text { Farmaci (alcool, estrogeni, estradiolo, androgeno, testosterone, glucocorticoide, } \\
\text { ciclosporina, tacrolimus, diuretico tiazidico, } \beta \text { bloccanti, inibitori delle proteasi, } \\
\text { sertralina, valproato) }\end{array}$ \\
\hline $\begin{array}{l}\text { Iperlipidemia familiare combinata (fenotipo IIb) } \\
\beta \text {-lipoproteinemia allargata (fenotipo III) }\end{array}$ & $\begin{array}{l}\text { Obesità centrale con insulino resistenza } \\
\text { Ipotiroidismo } \\
\text { Sindrome nefrosica e insufficienza renale cronica } \\
\text { Scorrette abitudini alimentari (dieta ad elevato contenuto di carboidrati } \\
\text { e eccessivo consumo di alcolici) }\end{array}$ \\
\hline
\end{tabular}


mia, e l'idiopatica iperchilomicronemia (idiopathic hyperchylomicronemia). Spesso vengono scoperte casualmente ad esami ematici di routine rendendo talvolta impossibile il dosaggio di molti parametri ematochimici. Il sangue prelevato appare lattescente se la trigliceride mia è superiore a $1000 \mathrm{mg} / \mathrm{dL}$. L'iperchilomicronemia primitiva è una rara malattia prevalentemente infantile, caratterizzata da pancreatite e dovuta a un'attività bassa/assente dell'enzima lipasi. Le forme più comuni di iperchilomicronemia sono tuttavia quelle dell'adulto, frequentemente secondarie a scompenso glicemico. Spesso è presente un'anomalia del metabolismo lipidico sottostante, per esempio un difetto eterozigote di lipoprotein lipasi o un'iperlipemia familiare combinata: l'interazione gene-ambiente determina in questi casi l'età di insorgenza e la gravità della malattia Le complicanze cliniche dell'iperchilomicronemia sono particolarmente frequenti per livelli di trigliceridemia superiori a 2000 $\mathrm{mg} / \mathrm{dL}$. In passato l'ipertrigliceridemia era considerata complicanza della pancreatite, ma più modernamente viene interpretata come sua determinante patogenetica. Il paziente adulto con sangue lattescente dovrebbe ricevere un attento esame obiettivo (ricerca di epato-splenomegalia e xantomatosi eruttiva, esame del fondo dell'occhio alla ricerca della lipemia retinalis) ed essere interrogato sull'utilizzo di farmaci potenzialmente implicati ( $\beta$-bloccanti, diuretici, estroprogestinici, cortisonici, resine a scambio ionico, acido retinoico e derivati, interferoni, inibitori delle proteasi, chemioterapici, immunosoppressori, ecc.). La determinazione della glicemia o della glicosuria (anche con uno stick urinario) rappresenta un esame fondamentale poiché può indirizzare verso una forma secondaria a diabete. L'ulteriore diagnostica di laboratorio è riservata a pochi centri specializzati. In considerazione dell'elevato rischio di pancreatite, l'iperchilomicronemia è l'unica forma di iperlipoproteinemia che richiede un trattamento urgente. La terapia è prevalentemente dietetica (correzione di eventuali eccessi, abolizione di alcol, alimentazione polipi dica, utilizzo di trigliceridi a media catena) e farmacologica (fibrati e acidi grassi omega-3). Talora può essere impiegata la plasmaferesi per periodi brevi o prolungati. ${ }^{5}$

\section{Management di un paziente con ipertrigliceridemia}

La valutazione del paziente con ipertrigliceridemia dovrebbe prevedere i seguenti steps (Figura 1): ${ }^{4}$

- Accurata anamnesi familiare volta a stabilire la presenza di malattia aterosclerotica prematura. Quindi le domande saranno volte a stabilire la presenza di patologie cardiovascolari in un parente di primo grado e l'età di insorgenza delle stesse. Una storia familiare di malattia coronarico prematura suggeri- sce la presenza di una ipertrigliceridemia secondaria a iperlipidemia familiare combinata o una ipo- $\alpha$-lipoproteinemia familiare.

- Escludere cause di ipertrigliceridemia secondarie (Tabella 2): valutazione compenso glicemico, studio della funzionalità tiroidea, studio della funzionalità surrenalica, valutazione funzionalità renale ed esame urine microscopico con particolare attenzione ai farmaci assunti.

- Calcolare l'indice di massa corporea con valutazione della circonferenza addominale. La combinazione di elevati trigliceridi con un'aumentata circonferenza addominale $(>101 \mathrm{~cm}$ nell'uomo e $>89 \mathrm{~cm}$ nella donna) può indirizzare verso una forma secondaria ad obesità centrale conseguente ad insulino-resistenza. Tra le forme genetiche può orientare verso una diagnosi di un'iperlipidemia familiare combinata (fenotipo IIb) o una ipo- $\alpha-$-lipoproteinemia familiare (fenotipo I) piuttosto che verso una forma una ipertrigliceridemia familiare (che non è associata ad adiposità centrale o ad un aumentato rischio cardiovascolare).

- Valutare i livelli di colesterolo HDL e LDL per poter distinguere tra una forma di ipertrigliceridemia familiare (LDL e HDL generalmente nella norma), una forma di iperlipidemia combinata (aumentati livelli di LDL e ridotti livelli di HDL) e una forma di ipo- $\alpha$-lipoproteinemia familiare (ridotti livelli di HDL).

- Valutare in pazienti con elevati livelli di trigliceridi e una storia anamnestica personale e familiare negativa per malattia coronarica i livelli di apolipoproteina $\mathrm{B}$ per poter distinguere tra una forma di iperlipidemia familiare combinata (aumentati) e una forma di ipertrigliceridemia familiare (ridotti). ${ }^{6,7}$

\section{Trattamento ipertrigliceridemia}

Dopo il trattamento delle forme secondarie eventualmente responsabili dell'ipertrigliceridemia i pazienti a rischio per malattia coronarica prematura devono essere trattati attraverso la modifica degli stili di vita e l'avvio di terapia farmacologica.

L'efficacia delle modifiche dello stile di vita sui livelli di $\mathrm{T}$ è ben documentata. A tutti i pazienti con obesità, sindrome metabolica o diabete mellito di tipo 2 dovrebbero essere obbligatoriamente raccomandati riduzione del peso insieme a un programma di regolare attività fisica di intensità moderata, che possono ridurre la trigliceridemia del 20-30\%. Rispetto ad una dieta ricca di grassi saturi, una ricca di grassi monoinsaturi migliora in modo significativo la sensibilità all'insulina. Ciò va in parallelo ad una riduzione dei livelli di trigliceridi, in particolare nel periodo postprandiale. L'effetto dannoso di una dieta ricca di carboidrati può essere ridotto se il loro assorbimento viene rallentato, quindi una dieta ricca di carboidrati 
ad elevato indice glicemico e povera di fibre influenza negativamente i livelli di trigliceridi. ${ }^{8}$

L'uso di farmaci per abbassare i $\mathrm{T}$ dovrebbe essere preso in considerazione solo in soggetti con trigliceridemia $>200 \mathrm{mg} / \mathrm{dL}$ che persiste dopo il tentativo di modifica dello stile di vita. I presidi farmacologici di- sponibili sono le statine, i fibrati, l'acido nicotinico e gli acidi grassi omega-3. La somministrazione di acidi grassi della serie omega-3 porta ad una riduzione significativa della trigliceridemia influenzando dunque i livelli di uno dei principali fattori di rischio lipidico per malattie vascolari. Dal punto di vista del farmaco-

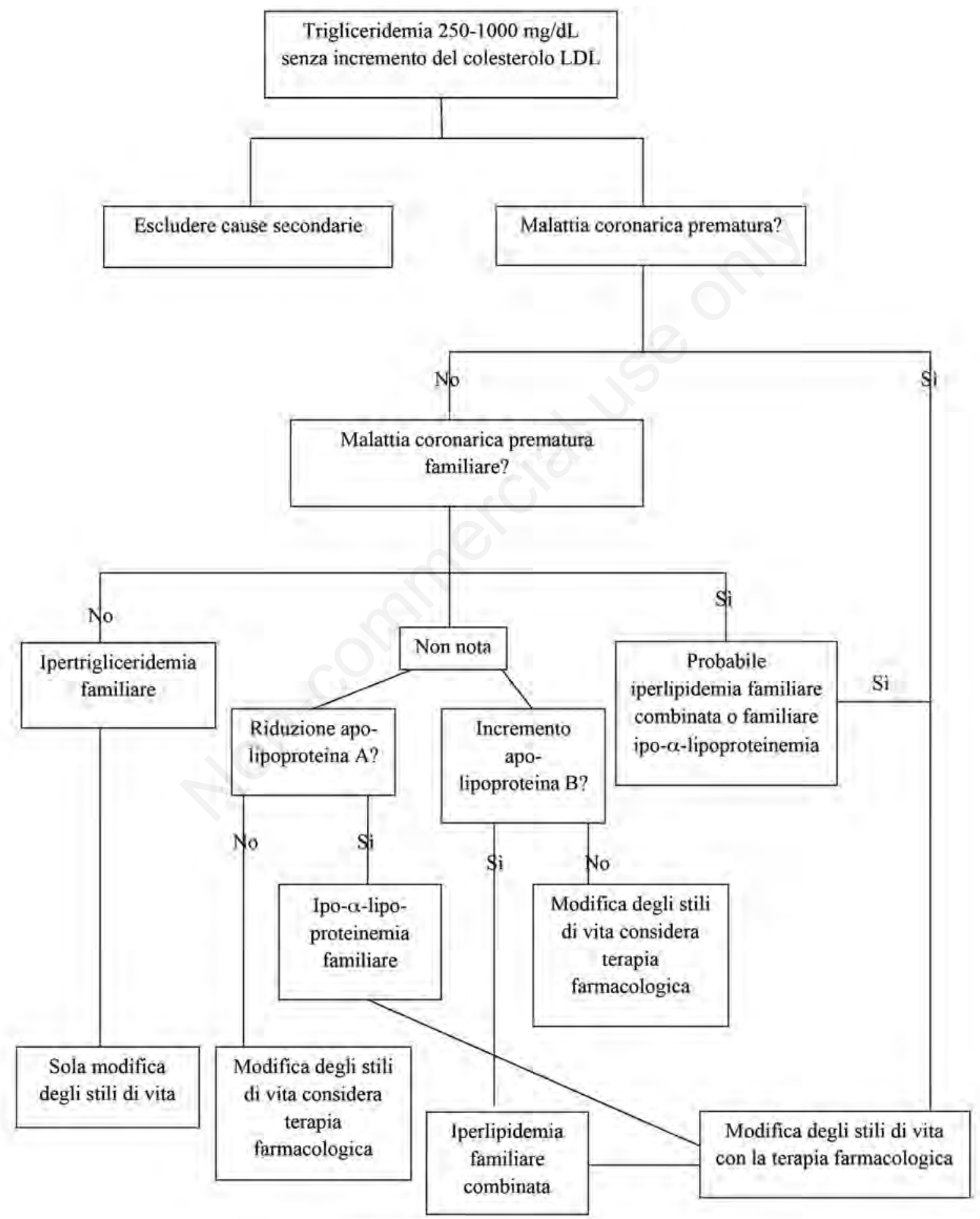

Figura 1. Algoritmo diagnostico per il management di un paziente con moderata ipertrigliceridemia. LDL, $\beta$-lipoproteine. 
logo la comprensione del meccanismo attraverso il quale questo farmaco agisce è di grande rilevanza per potere individuare le condizioni di alterato metabolismo nelle quali il farmaco sarà più efficace e potere, quindi, suggerire al medico quali siano i pazienti che più beneficeranno di questo trattamento. Studi sperimentali e clinici hanno documentato che gli acidi grassi omega-3 sopprimono la produzione epatica di lipoproteine ricche in trigliceridi e ne accelerano, in parte, la rimozione. L'azione sulla produzione dei trigliceridi avviene in massima parte a livello dell'epatocita ed è legata ad una ridotta produzione di apolipoproteina B, la parte proteica delle VLDL. Ne consegue una ridotta immissione in circolo di trigliceridi. Per quanto riguarda l'aumentata rimozione dei trigliceridi (quindi delle VLDL) è da correlarsi ad un incremento dell'attività della lipasi lipoproteica, enzima localizzato a livello dell'endotelio vascolare che risulta essere il principale determinante della rimozione dei trigliceridi dal circolo in periferia. Non a caso soggetti con deficit parziale di attività della lipasi lipoproteica sono ipertrigliceridemici. Poiché l'ipertrigliceridemia che si osserva normalmente a digiuno si esplica attraverso alterazione o della secrezione o del catabolismo dei trigliceridi, gli acidi grassi omega3 sono in grado di ridurre i livelli elevati di trigliceridi nella grande maggioranza dei casi di ipertrigliceridemia endogena. Tuttavia una popolazione specifica che beneficerà in modo particolare è quella rappresentata dai pazienti affetti da iperlipidemia familiare combinata, condizione di alto rischio cardiovascolare che è presente nella popolazione generale in circa un soggetto su 200. Questi pazienti hanno un fenotipo spesso caratterizzato da ipertigliceridemia ed un profilo lipoproteico altamente aterogenico conseguente ad un marcato aumento della produzione di apo B epatica: sono quindi il bersaglio ideale per una terapia con acidi grassi della serie omega-3. L'utilizzo degli acidi grassi a lunga catena omega-3 (PUFA n-3), o polinsaturi, (sempre congiuntamente alle misure non farmacologiche) è da considerarsi in caso di: i) ipertrigliceridemie elevate con inadeguata risposta alla terapia non farmacologica; ii) ipertrigliceridemie molto elevate con inadeguata risposta alla terapia non farmacologica. Nell'ipertrigliceridemia elevata l'obiettivo primario è la prevenzione cardiovascolare (CV); in primo luogo dovranno essere implementati i provvedimenti già previsti dalle linee guida (misure non farmacologiche, controllo delle dislipidemie con statine quando indicato, controllo ipertensione arteriosa quando indicato) e, se persiste rischio elevato, si possono aggiungere i PUFA. In questo caso, in analogia a quanto già previsto per la prevenzione secondaria, il dosaggio dovrebbe essere di una capsula al giorno. Nell'ipertrigliceridemia molto elevata l'obiettivo è la prevenzione primaria $\mathrm{CV}$ per i pazienti ad alto rischio (alcune forme familiari, diabete, sindrome metabolica, ecc.), con le stesse modalità sopra riportate. La prevenzione della pancreatite è invece l'obiettivo primario in presenza di trigliceridi $>1000 \mathrm{mg} / \mathrm{dL}$ e l'intervento prevede rigorose misure non farmacologiche e dosaggio di PUFA fino a 3 capsule al dì. In tutte queste indicazioni, i fibrati possono rappresentare un'alternativa (con l'attenzione del caso se si considera l'associazione con statine) e sono generalmente il farmaco di prima scelta nelle linee guida americane; è possibile l'associazione fibrati-PUFA nelle forme d'ipertrigliceridemia molto elevata. Può essere utile ricordare che aglio, farmaci alternativi o di erboristeria non hanno, attualmente, sufficienti prove di efficacia per essere utilizzati in questi casi. ${ }^{9}$

\section{Ipercolesterolemia}

\section{Ipercolesterolemia familiare (familial hypercholesterolemia)}

L'ipercolesterolemia familiare $(\mathrm{FH})$ è un comune disordine genetico del metabolismo lipidico, a trasmissione autosomica dominante e generalmente a penetranza completa, che si caratterizza per LDL-c e per incrementato rischio di malattia coronarica prematura. ${ }^{5}$ Gli individui affetti presentano un elevato rischio di eventi cardiovascolari con morte precoce nel $50 \%$ degli uomini e nel 15\% delle donne. Diverse sono le mutazioni responsabili di malattia: più frequentemente è alterato il gene del recettore LDL, $L D L R$; altre possibili mutazioni coinvolgono il gene dell'apolipoproteina $\mathrm{B}$, l'apo $B$, e della proproteina convertasi subtilisina/kexina 9, PCSK9. L'ipercolesterolemia familiare nella sua forma eterozigote $(\mathrm{HeFH})$ è verosimilmente la più frequente malattia genetica metabolica con un'incidenza stimata di circa 620.000 individui ed una prevalenza di circa 1 su 300-500 individui, con punte, in alcune popolazioni, di 1 ogni 100 individui. I valori di colesterolo LDL sono correlati alla severità della mutazione genetica e quindi conseguenti alla sua omozigosi o eterozigosi; valori compresi tra 200-400 mg/dL sono suggestivi per una $\mathrm{HeFH}$, mentre valori $>600 \mathrm{mg} / \mathrm{dL}$ per una forma omozigote. La forma eterozigote si caratterizza per la presenza di un gene normalmente funzionante e quindi un decorso meno aggressivo e una maggior risposta al trattamento con statina. L'età di sviluppo di malattia cardiovascolare in soggetti non trattati è 42-46 anni per gli uomini e 51-56 anni per le donne. Questa alterazione metabolica è responsabile di più del $50 \%$ di eventi cardiaci coronarici negli uomini di età $>50$ anni e nel $30 \%$ nelle donne di età $>60$ anni. La completa assenza di funzionalità del recettore per le LDL configura, invece, la forma omozigote. La malattia è spesso non responsiva al trattamento con statine e l'in- 
sorgenza di malattia cardiovascolare è estremamente precoce attestandosi intorno ai 20 anni. Nella maggior parte dei casi, la FH non viene diagnosticata se non dopo la comparsa del primo evento coronarico. ${ }^{10}$

La precoce identificazione degli individui affetti è molto importante perché un precoce trattamento farmacologico aggressivo può ridurre il rischio CV. I criteri diagnostici dell'ipercolesterolemia familiare includono elevati valori di colesterolo, presenza di xantomi tendinei nel pazienti e nei parenti di primo grado, familiarità o anamnesi personale positiva per malattia coronarica prematura o per elevati livelli di colesterolo. ${ }^{11}$

In Tabella 3 sono mostrati le raccomandazioni per la diagnosi ed i criteri diagnostici per $\mathrm{FH}^{2}$ e i valori di LDL che inducono la probabilità diagnostica di FH all' $80 \%{ }^{2}$

\section{Terapia}

Il primo approccio al paziente dislipidemico prevede la valutazione del suo rischio cardiovascolare totale, sulla base del quale verrà scelta la terapia farmacologica più appropriata. ${ }^{12} \mathrm{E}$ fondamentale considerare a rischio molto elevato o elevato tutti i pazienti affetti da: i) malattia cardiovascolare nota; ii) diabete mellito tipo II o tipo I con microalbuminuria; iii) malattia renale cronica.

Per gli altri pazienti, che non rientrano nelle suddette categorie, viene raccomandato l'utilizzo di tabelle di valutazione del rischio come quelle del sistema SCORE (che stima il rischio di un evento cardiovascolare fatale a 10 anni).
I trattamenti a disposizione per la cura dell'ipercolesterolemia sono 5 e sono illustrati nei seguenti sottoparagrafi.

\section{Statine}

Si tratta di molecole che agiscono inibendo l'attività dell'enzima HMG-CoA reduttasi determinando una riduzione della concentrazione intracellulare di colesterolo; a ciò fa seguito una maggiore espressione dei recettori per LDL $(L D L R)$ sulla superficie degli epatociti con conseguente maggiore sequestro di colesterolo LDL circolante e di altre lipoproteine contenenti apo B, tra cui le particelle ricche di trigliceridi.

Le statine sono i farmaci ipocolesterolemizzanti più studiati per la prevenzione del rischio cardiovascolare. Sono numerosi i trials clinici in cui è stata dimostrata la riduzione di morbilità e mortalità cardiovascolare sia in prevenzione primaria che secondaria. ${ }^{12}$

Le diverse statine differiscono per assorbimento, biodisponibilità, solubilità, escrezione. Alcune sono pro-farmaci (lovastatina e simvastatina), mentre altre vengono assunte direttamente nella loro forma attiva. È stata osservata, inoltre, una variabilità interindividuale alla risposta terapeutica, così come una diversa incidenza di effetti avversi. Dal momento che, alla dose massima, le diverse molecole differiscono per la loro capacità di ridurre i livelli di colesterolo LDL, nella pianificazione di un'efficace terapia con statine, può essere utile procedere in questo modo: ${ }^{13}$ i) valutare il rischio cardiovascolare totale del paziente; ii) coinvolgere il paziente nelle decisioni riguardanti la

Tabella 3. Raccomandazioni per la diagnosi di ipercolesterolemia familiare e livelli di colesterolo $\beta$-lipoproteine che ne inducono il sospetto clinico.

\begin{tabular}{ll}
\hline Raccomandazioni & Livelli di colesterolo LDL che inducono il sospetto clinico di FH \\
\hline $\begin{array}{l}\text { Lo screening per la ricerca di elevati valori sierici di colesterolo } \\
\text { è raccomandato in tutti i soggetti all'età di } 20 \text { anni }\end{array}$ & c-LDL $\geq 250 \mathrm{mg} / \mathrm{dL}$ in pazienti con età $\geq 30$ anni \\
\hline FH dovrebbe essere sospettata per il riscontro dei seguenti valori & c-LDL $\geq 220 \mathrm{mg} / \mathrm{dL}$ in pazienti con età tra 20-29 anni \\
di LDL colesterolo o colesterolo non HDL: \\
- adulti (età $\geq 20$ anni): LDL colesterolo $\geq 190 \mathrm{mg} / \mathrm{dL}$ \\
$\quad$ o colesterolo non HDL $\geq 220 \mathrm{mg} / \mathrm{dL}$ \\
- adolescenti o soggetti con età inferiore a 20 anni: \\
$\quad$ c-LDL $\geq 160 \mathrm{mg} / \mathrm{dL}$ o colesterolo non $\mathrm{HDL} \geq 190 \mathrm{mg} / \mathrm{dL}$
\end{tabular}

Tutti gli individui con il riscontro di questi valori devono essere c-LDL $\geq 190 \mathrm{mg} / \mathrm{dL}$ in pazienti con età $<20$ anni

indagati per un'anamnesi familiare positiva per malattia

coronarica prematura

La colesterolemia deve essere dosata già all'età di 2 anni nei

bambini con una storia familiare di malattia coronarica prematura

Sebbene non presenti in molti soggetti con FH, i seguenti reperti

clinici dovrebbero indurre il clinico a sospettare la diagnosi di $\mathrm{FH}$ :

- xantomi tendinei ad ogni età

- xantelasmi in pazienti con età $<20$ anni

- cornea arcuata in pazienti con età $<45$ anni

FH, ipercolesterolemia familiare; LDL, $\beta$-lipoproteine; c-LDL, lipoproteine a bassa densità; HDL, $\alpha$-lipoproteine. 
gestione del rischio cardiovascolare; iii) identificare di un livello di colesterolo LDL target, a seconda del rischio stimato; iv) calcolare la riduzione percentuale di colesterolo LDL necessaria per raggiungere il target identificato; v) scegliere la statina con la quale ottenere la riduzione desiderata; vi) titolare il dosaggio di statina al fine di raggiungere il target; vii) se non efficace, considerare di una combinazione di farmaci.

Gli effetti avversi sono a carico del muscolo (mialgie con possibile progressione verso la rabdomiolisi) ed incremento dose-dipendente degli enzimi epatici. I possibili effetti metabolici (aumento del rischio di incidenza di diabete mellito) vanno valutati nell'ambito del profilo di rischio del paziente; per soggetti ad alto rischio, la riduzione di eventi cardiovascolari sembra prevalere sul lieve incremento di incidenza del diabete.

\section{Inibitori dell'assorbimento di colesterolo}

L'ezetimibe agisce inibendo l'assorbimento di colesterolo esogeno a livello della mucosa intestinale; in risposta alla riduzione di colesterolo che giunge al fegato, viene aumentata l'esposizione di $L D L R$ e, di contro, si riduce il colesterolo circolante.

Ezetimibe è efficace nel ridurre i livelli di colesterolo LDL sia in monoterapia (con riduzioni del $15-22 \%$ rispetto ai valori di base) sia, soprattutto, in associazione con statine, dal momento che l'effetto di queste ultime tende ad aumentare l'assorbimento di colesterolo a livello intestinale. L'ezetimibe in associazione con una statina può determinare un'ulteriore riduzione di colesterolo LDL del $15-20 \%$, indipendentemente dalla statina utilizzata e dalla sua posologia. È indicato come terapia di seconda linea in associazione con statina laddove non venga raggiunto il target terapeutico stabilito oppure in monoterapia in pazienti intolleranti alle statine o per i quali esse siano controindicate. Non è gravato da effetti collaterali particolari al di fuori di mialgie e moderato incremento di enzimi epatici. ${ }^{12}$

\section{Resine sequestranti gli acidi biliari (colestiramina e colesevelam)}

Le resine sequestranti gli acidi biliari, come colestiramina e colesevelam, sono sostanze che non vengono assorbite né modificate nel lume intestinale. Agiscono legando gli acidi biliari, prevenendone l'assorbimento e riducendone il ricircolo enteroepatico; la riduzione degli acidi biliari determina un aumento del catabolismo del colesterolo epatico (per sintetizzare nuovi acidi biliari) ed un aumento compensatorio dell'attività epatica del recettore per LDL $(L D L R)$ con conseguente aumentato sequestro di colesterolo LDL dal circolo. Un effetto parallelo è la riduzione della glicemia, utile nei pazienti iperglicemici. Gli effetti metabolici si esplicano sui livelli di colesterolo LDL, senza effetti sul c-HDL; in alcuni pazienti i trigliceridi possono aumentare.
Colesevelam è meglio tollerato rispetto a colestiramina. Gli effetti collaterali si possono presentare anche a bassi dosaggi e sono costituiti per lo più da flatulenza, stipsi, nausea e dispepsia. ${ }^{12}$

\section{Aferesi delle $\beta$-lipoproteine}

Si tratta di una procedura molto costosa, eseguita in centri specializzati ed indicata in particolare per $i$ rari pazienti con dislipidemia severa, soprattutto affetti da ipercolesterolemia familiare severa eterozigote o omozigote, nei quali anche le terapie farmacologiche di associazione non sono sufficienti al raggiungimento dei target terapeutici. Il paziente viene posto in circolazione extracorporea, settimanalmente o a settimane alterne, con rimozione plasmatica di LDL e Lp(a). ${ }^{12}$

Nelle forme di ipercolesterolemia familiare le statine rimangono il caposaldo della terapia; molto spesso, tuttavia, la monoterapia non è sufficiente per raggiungere $\mathrm{i}$ target di colesterolo LDL ed è richiesta la terapia di associazione con ezetimibe e sequestranti gli acidi biliari o, addirittura, la aferesi delle LDL. ${ }^{14}$

\section{Nuove prospettive}

Una nuova classe di farmaci, gli inibitori della proproteina convertasi subtilisina/kexina tipo 9 (PCSK9), si sta affacciando sul panorama degli ipocolesterolemizzanti. Al momento della stesura di questa monografia, in Europa evolocumab, anticorpo monoclonale a somministrazione iniettiva, ha ricevuto l'indicazione al trattamento delle ipercolesterolemie familiari eterozigoti $(\mathrm{HeFH})$ e delle dislipidemie miste in terapia con la dose massima tollerata di statine o altro ipolipemizzante non in grado di raggiungere il target terapeutico. L'indicazione si estende anche ai casi di pazienti che non tollerano le statine o per i quali siano controindicate. ${ }^{15}$

\section{Iperlipidemia familiare combinata (familial combined hyperlipidemia)}

L'iperlipidemia familiare combinata $(\mathrm{FCH})$ è un disordine metabolico caratterizzato da: i) incremento del colesterolo e dei trigliceridi in almeno due membri della stessa famiglia; ii) variabilità intra-individuale e intra-familiare del fenotipo lipidico; iii) incrementato rischio di malattia coronarica prematura. ${ }^{6,16}$

Sinonimi di questa sindrome sono le alterazioni metaboliche definite come iperlipidemia familiare a fenotipo multiplo, iperlipidemia familiare mista, iperlipoproteinemia familiare combinata e ipercolesterolemia-ipertrigliceridemia familiare combinata .

Questa sindrome è estremamente comune ed è considerata una tra le più comuni forme genetiche di iperlipidemia con una prevalenza di circa lo $0,5-2 \%$ nella popolazione. In Europa sono pertanto affetti da questa sindrome circa 3,5 milioni di soggetti. È presente nel $10 \%$ dei pazienti affetti da malattia coronarica e 
nell' $11 \%$ circa dei pazienti con infarto miocardico ed età $<60$ anni.

Le alterazioni metaboliche di questa sindrome includono un incremento dei trigliceridi plasmatici e/o del colesterolo con alti livelli di lipoproteine VLDL e/o LDL e di apolipoproteina B 100 (>125 mg/dL). Alcuni pazienti possono inoltre presentare ridotti livelli di lipoproteine ad alta densità, HDL, spesso inversamente correlate ai livelli di trigliceridi. La Tabella 4 riporta i criteri diagnostici per questa sindrome. ${ }^{7}$

Una volta posta la diagnosi il paziente dovrebbe essere sottoposto all'esecuzione di un Doppler dei tronchi sovra-aortici per valutare la presenza di eventuali placche aterosclerotiche oltre allo studio dell'aorta addominale e delle arterie iliache-femorali. La recente codifica dei criteri diagnostici per la sindrome metabolica (SM) ha determinato un significativo overlap con la diagnosi di FCH. La presenza di sindrome metabolica è identificata nel $65 \%$ dei pazienti con FCH rispetto al $19 \%$ nel controllo senza FCH. L'incrementata prevalenza di SM nei pazienti con FCH potrebbe in parte spiegare l'aumentato rischio CV tipico dei soggetti affetti. In Tabella 5 sono presentati le principali caratteristiche comuni e le differenze tra FCH e SM. ${ }^{7}$
Dal punto di vista terapeutico l'associazione di una statina con farmaci attivi sui trigliceridi (omega 3, fibrati, acido nicotinico) è il trattamento di scelta.

In assenza di dati prognostici a lungo termine sulle possibili strategie terapeutiche i pazienti affetti da FCH devono essere monitorati non solo dal punto di vista laboratoristico ma anche dal punto di vista strumentale e clinico in merito all'aumentato rischio cardio-vascolare.

\section{Iperlipidemia lipoproteina-a}

La lipoproteina-a, descritta per la prima volta da Berg nel 1963, è una lipoproteina distinta dalle LDL da un'apoproteina, apo A, collegata all'apo B da un legame disulfide. Il gene dell'apo (a) (LPA) è il maggior determinante della concentrazione plasmatica di Lp (a). Questa sindrome si caratterizza per elevati livelli di Lp (a) emerso in vari studi come fattore di rischio indipendente di malattia cardiovascolare. ${ }^{14}$ Sono state descritte numerose varianti genetiche di questo gene. Attualmente non esiste un trattamento farmacologico approvato per ridurre i livelli di $\mathrm{Lp}$ (a). La prevenzione e/o l'adeguato trattamento dei fattori di rischio per malattia CV come il fumo, l'ipertensione, il diabete, l'ipotiroidismo e il consumo di alcolici sem-

Tabella 4. Criteri diagnostici dell'iperlipidemia familiare combinata.

\begin{tabular}{ll}
\hline Diagnosi di I livello & Diagnosi di II livello (solo centri specializzati) \\
\hline Ipercolesterolemia LDL $>160 \mathrm{mg} / \mathrm{dL}$ e/o trigliceridi $>200 \mathrm{mg} / \mathrm{dL}$ & $\begin{array}{l}\text { Valutazione del livello plasmatico di apo B } 100 \text { con metodo } \\
\text { immunoturbidometrico }\end{array}$ \\
\hline $\begin{array}{l}\text { Presenza di ipercolesterolemia, ipertrigliceridemia o entrambe } \\
\begin{array}{l}\text { in un membro della famiglia valutato in almeno 3 prelievi } \\
\text { consecutivi (bimensili) }\end{array}\end{array}$ & Ricerca delle particelle LDL piccole e dense \\
\hline
\end{tabular}

Test genetico per escludere altre forme di dislipidemia familiare

LDL, $\beta$-lipoproteine.

Tabella 5. Differenze tra iperlipidemia familiare combinata e sindrome metabolica.

\begin{tabular}{|c|c|}
\hline Manifestazioni comuni & Principali differenze \\
\hline Frequente ipertrigliceridemia e/o bassi livelli di colesterolo HDL & $\begin{array}{l}\text { Apo B è aumentato in paziente con FCH ma non in pazienti con } \\
\text { sindrome metabolica } \\
\text { I valori di LDL sono usualmente normali o inferiori in SM }\end{array}$ \\
\hline $\begin{array}{l}\text { Frequente associazione con fattori di rischio cardiovascolare come } \\
\text { l'ipertensione, l'obesità addominale, diabete/ridotta tolleranza } \\
\text { ai carboidrati }\end{array}$ & $\begin{array}{l}\text { Il fenotipo lipidico è maggiormente variabile (sia nel singolo individuo } \\
\text { che nella famiglia) nei pazienti con FCH rispetto ai pazienti con SM }\end{array}$ \\
\hline Notevole aumento del rischio cardiovascolare & $\begin{array}{l}\text { La modifica dello stile di vita è meno rilevante dal punto di vista } \\
\text { dell'efficacia nel paziente con FCH rispetto al paziente con SM } \\
\text { Il basso grado di infiammazione (alti livelli plasmatici di molecole di } \\
\text { adesione) e/o uno stato pro-coagulativo (alti livelli plasmatici di } \\
\text { fibrinogeno e PAI-1) sono più frequentemente associati con SM } \\
\text { Le alterazioni cliniche e laboratoristiche sono più precoci in FCH } \\
\text { rispetto ai pazienti con SM }\end{array}$ \\
\hline
\end{tabular}

HDL, $\alpha$-lipoproteine; FCH, iperlipidemia familiare combinata; LDL, $\beta$-lipoproteine; SM, sindrome metabolica; PAI-1, inibitore-1 dell'attivatore del plasminogeno. 
bra essere la strategia al momento più efficace. L'HEART-UK working group raccomanda in pazienti con concentrazioni plasmatiche di $\mathrm{Lp} \mathrm{(a)}>60 \mathrm{mg} / \mathrm{dL}$ e di LDL colesterolo $>130 \mathrm{mg} / \mathrm{dL}$ (a dispetto della terapia con statina a dosaggio massimale) il trattamento aferetico. ${ }^{17}$

\section{Malattia della larga banda $\beta$ (broad $\beta$ band)}

I trigliceridi circolanti sono in parte esogeni (originati dai grassi dietetici), trasportati nei chilomicroni, e in parte endogeni (di sintesi epatica), trasportati nelle VLDL. Nei capillari dei muscoli e del tessuto adiposo le lipoproteine ricche in trigliceridi (chilomicroni e VLDL) sono idrolizzate dalla LPL ad acidi grassi liberi. I trigliceridi ed il colesterolo sintetizzati nel fegato o provenienti dal ciclo esogeno vengono esportati nel sangue sotto forma di VLDL $\left(\mathrm{d}<1,006 \mathrm{~g} / \mathrm{cm}^{3}\right)$, le cui apolipoproteine più importanti sono apo B-100 (513 kDa), apo E e apo C-II. Le VLDL vanno incontro allo stesso processo di distacco dei chilomicroni, per azione della LPL presente sulla superficie dei capillari. Tale processo rifornisce di acidi grassi il tessuto adiposo. Ciò porta alla formazione di IDL $\left(1,006<\mathrm{d}<1,019 \mathrm{~g} / \mathrm{cm}^{3}\right)$, ricche in colesterolo esterificato: il $25 \%$ viene ricaptato dal fegato, ad opera dei recettori per le LDL, la quota rimanente va incontro ad ulteriore perdita di trigliceridi, con formazione di $\operatorname{LDL}\left(1,019<\mathrm{d}<1,063 \mathrm{~g} / \mathrm{cm}^{3}\right)$, ancora più arricchite di colesterolo esterificato endogeno. ${ }^{18}$ Secondo la classificazione del National Cholesterol Education Program Adult Treatment Panel III (NCEP ATP III) i trigliceridi sono normali quando inferiori a 150 $\mathrm{mg} / \mathrm{dL}$, si definiscono borderline tra 150 e $199 \mathrm{mg} / \mathrm{dL}$, elevati tra 200 e $499 \mathrm{mg} / \mathrm{dL}$ e molto elevati al di sopra di $500 \mathrm{mg} / \mathrm{dL} .{ }^{19} \mathrm{La}$ prevalenza dell'ipertrigliceridemia è di circa il $30 \%$ negli adulti di età superiore a 20 anni e sale al $43 \%$ al di sopra dei 50 anni. Nell'ipertrigliceridemia di primo riscontro occorre verificare le condizioni preanalitiche, in particolare la relazione temporale tra prelievo e pasto e le caratteristiche qualitative del pasto. Dopo l'assunzione di cibo i trigliceridi restano elevati nel plasma per almeno 8 ore e in questo arco di tempo il valore rilevato non è rappresentativo del valore di riferimento del paziente. In considerazione di tale cinetica la valutazione del profilo lipidico va eseguita in condizioni di digiuno da 12 ore. Il dato risente della composizione del pasto e correla con il suo contenuto in grassi. La disbetalipoproteinemia, definita anche iperlipoprotinemia di tipo III secondo Frederickson o malattia della larga banda $\beta$, è una forma rara a trasmissione autosomica recessiva, dovuta ad una mutazione del gene che codifica la sintesi dell'apo E. La sostituzione aminoacidica dell'apo E causa una meno efficiente captazione epatica dei chilomicroni e delle IDL per carenza o inattività di apo
E-3 con riduzione della clearance dei remnants..$^{20} \mathrm{E}$ caratterizzata da ipercolesterolemia e ipertrigliceridemia da accumulo di residui di VLDL e chilomicroni, ricchi in esteri di colesterolo. Sul piano clinico si possono rilevare alterazioni cutanee proprie dell'ipercolesterolemia e dell'ipertrigliceridemia concomitanti: xantomi tuberosi e tendinei, xantomi striati palmari patognomonici (strie giallastre nelle pieghe interdigitali e sui palmi), xantelasmi, arco corneale. Comporta aterosclerosi precoce che si può manifestare clinicamente con infarto del miocardio, stroke o arteriopatia periferica a esordio giovanile ma in genere non infantile. ${ }^{21} \mathrm{~L}$ 'assetto lipidico è caratterizzato da incremento combinato di colesterolo e trigliceridi della stessa entità, entrambi pari a circa $400 \mathrm{mg} / \mathrm{dL}$ con rapporto $1 / 1$; la ricerca dell'omozigosi per apo E-2 supporta la diagnosi di certezza. Nel 10-20\% l'ipertrigliceridemia è secondaria a patologie il cui riconoscimento e trattamento è il primo obiettivo per la correzione dell'alterazione lipidica. Le cause delle forme secondarie di ipertrigliceridemia comprendono scorrette abitudini alimentari (eccessivo consumo di alcol, dieta a elevato contenuto di carboidrati), sindrome metabolica, diabete mellito di tipo II, gravidanza, obesità, ipotiroidismo, insufficienza renale cronica, sindrome nefrosica, sindrome di Cushing e assunzione di vari farmaci quali corticosteroidi, tiazidici, $\beta$-bloccanti, tamoxifene, estrogeni e contraccettivi orali, ciclosporina, acido valproico, antipsicotici recenti, inibitori delle proteasi usati nella terapia dell'infezione da HIV. Quando i trigliceridi superano i $1000 \mathrm{mg} / \mathrm{dL}$ il paziente è a rischio di pancreatite acuta, mentre valori superiori a $2000 \mathrm{mg} / \mathrm{dL}$ devono essere considerati e trattati come un'urgenza medica. In realtà livelli tanto elevati non sono comuni e inducono a pensare a una dislipidemia genetica. Il ruolo dell'ipertrigliceridemia come fattore di rischio cardiovascolare indipendente è invece controverso: non è chiaro se questa condizione possa provocare di per sé coronaropatia o sia soltanto un marker di altre alterazioni lipoproteiche direttamente causa di coronaropatia. ${ }^{22}$

In particolare, l'ipertrigliceridemia moderata è considerata un marker di dislipidemia aterogena e di sindrome metabolica poiché correla con la presenza di LDL piccole e dense e con ridotti livelli di colesterolo HDL, spesso si associa a un aumento di VLDL grandi e aterogene. Nella valutazione di un soggetto con ipertrigliceridemia, indipendentemente dalla causa, è necessario esplorare la presenza dei fattori di rischio cardiovascolari maggiori: fumo, ipertensione arteriosa (pressione arteriosa 140/90 mmHg o impiego di antipertensivi), età (45 anni nei maschi; 55 anni nelle femmine), storia familiare di cardiopatia ischemica precoce nei familiari di primo grado ( $<55$ anni nei maschi; $<65$ anni nelle femmine), basso colesterolo HDL $(<40$ $\mathrm{mg} / \mathrm{dL}$ ). Fondamentale è la stratificazione del rischio 
e la definizione degli obiettivi terapeutici primari e secondari per le diverse classi di rischio. La supplementazione con acidi grassi omega- 3 alla dose di almeno 4 $\mathrm{g} /$ die contribuisce a un'ulteriore riduzione dei trigliceridi fino al 30\% con diversi meccanismi d'azione: ridotta sintesi epatica di trigliceridi, aumento della loro ossidazione epatica, potenziamento dell'espressione tissutale. Affinché siano efficaci è necessaria una dose quotidiana alta, indicazione limitata in diversi pazienti dalla compliance terapeutica. I fibrati (gemfibrozil 600 $\mathrm{mg} 2$ volte/die, fenofibrato $145 \mathrm{mg} / \mathrm{die}$ ) riducono i trigliceridi del $20-50 \%$, con incremento del colesterolo HDL fino al $20 \%$ ed effetto variabile sul colesterolo LDL; qualitativamente si riducono le LDL piccole e dense aterogene. Hanno un complesso meccanismo d'azione che produce multipli effetti sulla via biosintetica e catabolica dei trigliceridi. Agiscono come attivatori del fattore di trascrizione nucleare PPAR- $\alpha$ (peroxisome proliferator-activated receptor- $\alpha$ ), coinvolto nel controllo di svariate funzioni cellulari, compresi il metabolismo glucidico e lipidico, la funzione endoteliale e l'infiammazione. I fibrati riducono la secrezione epatica di VLDL e incrementano la lipolisi plasmatica dei trigliceridi. L'aumento del colesterolo HDL dipende da aumentata espressione di apo A-1 e 2. Sono farmaci ben tollerati, di rado causano miosite o franca miopatia fino alla rabdomiolisi, in particolare se associati alle statine in pazienti con insufficienza renale. Possono favorire lo sviluppo di colelitiasi per incremento della concentrazione biliare di colesterolo. ${ }^{22}$

\section{Indicazioni alla rimborsabilità dei farmaci ipolipidemizzanti: la nota 13}

In Italia, l'Agenzia Italiana del Farmaco (AIFA) ha prodotto una serie di note limitative che assicurano l'appropriatezza di utilizzo dei farmaci e garantiscono, allo stesso tempo, uno strumento di governo della spesa farmaceutica. Le note AIFA sono indicazioni che si fondano sui risultati di sperimentazioni cliniche randomizzate e subiscono periodiche revisioni; permettono al medico la prescrizione di un farmaco a carico del Servizio Sanitario Nazionale (SSN). ${ }^{21}$

La nota 13 regolamenta la prescrizione a carico del SSN di statine (simvastatina, pravastatina, fluvastatina, lovastatina, atorvastatina, rosuvastatina), fibrati (bezafibrato, fenofibrato, gemfibrozil), omega 3, ezetimibe e resine sequestranti gli acidi biliari. ${ }^{23}$

In adesione alle linee guida europee della European Society of Cardiology/European Atherosclerosis Society (ESC/EAS) sul trattamento delle dislipidemie, viene stratificato in 5 categorie il rischio cardiovascolare a 10 anni, facendo riferimento alle carte del rischio SCORE per i pazienti senza evidenza di malattia. Rispetto alla versione precedente, la nuova nota 13 aggiornata al 2013 ha introdotto alcune novità quali la ridefinizione dei livelli di rischio nelle classi, l'identificazione della classe di rischio medio, con target di colesterolo LDL $<130 \mathrm{mg} / \mathrm{dL}$, la distinzione dei pazienti con insufficienza renale cronica (IRC) in due fasce di rischio (alto o molto alto) con distinti target terapeutici, la reintroduzione di lovastatina e fluvastatina e la definizione degli ambiti di prescrivibilità della rosuvastatina nei pazienti a rischio molto alto.

In una dettagliata tabella, alla categoria di rischio viene associato il relativo target terapeutico (TT) di colesterolo LDL e la proposta di trattamento rimborsabile con statine elo ezetimibe: ${ }^{23}$

- I pazienti che presentano risk score $<1 \%$ per malattia cardiovascolare fatale a 10 anni sono considerati a rischio basso e l'indicazione è alla sola modifica dello stile di vita.

- Pazienti con risk score 2-3\% sono a rischio medio: per costoro il trattamento farmacologico viene preso in esame solo dopo la modifica dello stile di vita per almeno 6 mesi; il TT di colesterolo LDL è $<130 \mathrm{mg} / \mathrm{dL}$

- Pazienti con rischio 4-5\% sono considerati a rischio moderato; il TT di colesterolo LDL è $<115 \mathrm{mg} / \mathrm{dL}$.

- Pazienti con risk score $\geq 5 \%$ e $<10 \%$ sono a rischio alto; da considerare a rischio alto anche i pazienti con dislipidemie familiari o con ipertensione severa, i pazienti diabetici senza fattori di rischio cardiovascolare e senza danno d'organo ed i pazienti con IRC moderata (glomerular filtration rate [GFR] 30-59 $\mathrm{mL} / \mathrm{min} / 1,73 \mathrm{~m}^{2}$ ). Il TT di colesterolo LDL è $<100 \mathrm{mg} / \mathrm{dL}$.

- A rischio molto alto sono soggetti con risk score $\geq 10 \%$, pazienti con malattia coronarica, ictus ischemico, arteriopatie periferiche, pregresso infarto miocardico, by pass aorto-coronarico, pazienti diabetici con uno o più fattori di rischio cardiovascolare e/o marcatori di danno d'organo (come la microalbuminuria) oltre ai pazienti con IRC grave (GFR $15-29 \mathrm{~mL} / \mathrm{min} / 1,73 \mathrm{~m}^{2}$ ). Per questi pazienti il TT di colesterolo LDL è $<70 \mathrm{mg} / \mathrm{dL}$. La Tabella $6^{23}$ schematizza le indicazioni sulla rimborsabilità di statine ed ezetimibe, secondo quanto detto nel precedente paragrafo.

I fibrati, i PUFA n-3 e le resine sequestranti gli acidi biliari vengono rimborsati dal SSN secondo le indicazioni riportate in Tabella $7 .{ }^{23}$

La nota 13 dà, inoltre, indicazioni sui farmaci prescrivibili nei casi di iperlipidemie indotte da farmaci immunosoppressori, antiretrovirali ed inibitori della aromatasi, non corrette dalla sola dieta; sono a carico del SSN le statine, i fibrati nel caso in cui predomini l'ipertrigliceridemia e l'ezetimibe in monoterapia per pazienti che non tollerano le statine o non possono assumerle. Si raccomanda di porre attenzione alle interferenze con la terapia antiretrovirale, e di evitare, in questi pazienti, l'utilizzo di simvastatina. 
Tabella 6. La nota 13 AIFA. Sintesi sulla rimborsabilità delle statine (ed ezetimibe) in base al livello di rischio.

Risk score e comorbidità
Risk score $\geq 10 \%$
Malattia coronarica/by pass aorto-coronarico
Stroke ischemico
Arteriopatie periferiche
Pregresso IMA
Diabete mellito con uno o più fattori di rischio
CV e/o markers di danno d'organo
(come la microalbuminuria)
IRC grave (e-GFR $15-29 \mathrm{~mL} / \mathrm{min} / 1,73 \mathrm{~m}^{2}$ )

Dislipidemie familiari

Ipertensione severa

Diabete senza fattori di rischio $\mathrm{CV}$

e senza danno d'organo

IRC moderata (FG $30-59 \mathrm{~mL} / \mathrm{min} / 1,73 \mathrm{~m}^{2}$ )

\begin{tabular}{ll} 
Prescrizione a carico del SSN & Tipologia di farmaco \\
\hline Colesterolo LDL $>70 \mathrm{mg} / \mathrm{dL}$ & Atorvastatina \\
(obiettivo raccomandato $<70 \mathrm{mg} / \mathrm{dL})$ & Pravastatina \\
& Fluvastatina \\
& Lovastatina \\
& Simvastatina \\
& Rosuvastatina nei pazienti in cui ci sia stata
\end{tabular}

evidenza di effetti collaterali severi nei primi

6 mesi di terapia con altre statine

In caso d'intolleranza a tutte le statine è

rimborsato il trattamento con ezetimibe in

monoterapia

Nei pazienti con sindromi coronariche acute

$o$ in quelli sottoposti a interventi di

rivascolarizzazione percutanea è indicata

atorvastatina a dosaggio elevato $(>40 \mathrm{mg})$

Colesterolo LDL $>100 \mathrm{mg} / \mathrm{dL}$

(obiettivo raccomandato

$<100 \mathrm{mg} / \mathrm{dL}$ )

\section{Simvastatina}

Pravastatina

Fluvastatina

Lovastatina

Atorvastatina

Se necessaria riduzione $\mathrm{LDL}>50 \%$

preferire atorvastatina

In caso l'obiettivo non sia stato raggiunto

rosuvastatina o ezetimibe più statine

(in associazione estemporanea o precostituita)

In caso d'intolleranza a tutte le statine è

rimborsato il trattamento con ezetimibe in

monoterapia

\begin{tabular}{lll}
\hline Risk score $4 \%-5 \%$ & $\begin{array}{l}\text { Colesterolo LDL }>115 \mathrm{mg} / \mathrm{dL} \\
\text { (obiettivo raccomandato } \\
<115 \mathrm{mg} / \mathrm{dL})\end{array}$ & $\begin{array}{l}\text { Simvastatina } \\
\text { Pravastatina } \\
\text { Fluvastatina } \\
\text { Lovastatina } \\
\text { Atorvastatina }\end{array}$ \\
\hline Risk score $2 \%-3 \%$ & & Modifica dello stile di vita per almeno $6 \mathrm{mesi}$ \\
& Colesterolo LDL $>130 \mathrm{mg} / \mathrm{dL}$ & $\begin{array}{l}\text { Simvastatina } \\
\text { Pravastatina } \\
\text { Fluvastatina }\end{array}$ \\
& $<130 \mathrm{mg} / \mathrm{dL})$ & Lovastatina \\
Atorvastatina
\end{tabular}

Risk score $<1 \% \quad$ Mai $\quad$ Indicata solamente la modifica dello stile di vita

SSN, Sistema Sanitario Nazionale; IMA, infarto miocardico acuto; CV, cardiovascolare; IRC, insufficienza renale cronica; e-GFR, estimated glomerular filtration rate; LDL, $\beta$-lipoproteine; FG, filtrato glomerulare.

Tabella 7. Rimborsabilità dei fibrati, degli acidi grassi a lunga catena omega-3 e delle resine sequestranti gli acidi biliari secondo la nota AIFA.

\begin{tabular}{|c|c|c|}
\hline La rimborsabilità dei fibrati & La rimborsabilità di PUFA n-3 & $\begin{array}{l}\text { La rimborsabilità delle resine } \\
\text { sequestranti gli acidi biliari }\end{array}$ \\
\hline $\begin{array}{l}\text { Pazienti già in trattamento con statine che } \\
\text { presentino in trattamento: } \\
\text { i) livelli di } \mathrm{HDL} \text { colesterolo basso }(<40 \mathrm{mg} / \mathrm{dL} \\
\text { nei } \mathrm{M} \text { e } 50 \mathrm{mg} / \mathrm{dL} \text { nelle } \mathrm{F}) \\
\text { ii) e/o trigliceridi elevati }(>200 \mathrm{mg} / \mathrm{dL}) \\
\text { In questi casi il farmaco di prima scelta è il } \\
\text { fenofibrato per la maggiore sicurezza di uso } \\
\text { nei pazienti in terapia con statine; la combinazione } \\
\text { di statine e gemfibrozil è invece associata ad un } \\
\text { aumentato rischio di miopatia }\end{array}$ & $\begin{array}{l}\text { Iperlipemia familiare combinata } \\
\text { già in terapia con statine nel caso } \\
\text { permangono elevati i livelli } \\
\text { di trigliceridi }\end{array}$ & $\begin{array}{l}\text { Ipercolesterolemia familiare, nel caso le } \\
\text { statine più ezetimibe non siano } \\
\text { sufficienti }\end{array}$ \\
\hline $\begin{array}{l}\text { Disbetalipoproteinemia (per i trigliceridi elevati, } \\
\text { solitamente insieme a statine) }\end{array}$ & $\begin{array}{l}\text { Forme familiari con trigliceridi } \\
\text { molto elevati: iperchilomicronemie } \\
\text { e grave ipertrigliceridemia }\end{array}$ & $\begin{array}{l}\text { Disbetalipoproteinemia, nel caso } \\
\text { statine, più ezetimibe (e fibrati) } \\
\text { non siano sufficienti }\end{array}$ \\
\hline $\begin{array}{l}\text { Forme familiari con trigliceridi molto elevati: } \\
\text { iperchilomicronemie e gravi ipertrigliceridemie }\end{array}$ & $\begin{array}{l}\text { Pazienti con insufficienza renale } \\
\text { moderata e grave con trigliceridi }>500 \mathrm{mg} / \mathrm{dL}\end{array}$ & \\
\hline
\end{tabular}

PUFA n-3, acidi grassi a lunga catena omega-3. 
Tabella 8. La rimborsabilità dei farmaci prescrivibili nelle dislipidemie familiari.

\begin{tabular}{|c|c|c|c|}
\hline Patologia & Trattamento $1^{\circ}$ livello & Trattamento $2^{\circ}$ livello & Trattamento $3^{\circ}$ livello \\
\hline Ipercolesterolemia familiare & $\begin{array}{l}\text { Simvastatina } \\
\text { Pravastatina } \\
\text { Fluvastatina } \\
\text { Lovastatina } \\
\text { Atorvastatina } \\
\text { Rosuvastatina* }\end{array}$ & $\begin{array}{l}\text { Ezetimibe più statine } \\
\text { (in associazione estemporanea } \\
\text { o precostituita)* }\end{array}$ & $\begin{array}{l}\text { Aggiunta di resine sequestranti } \\
\text { gli acidi biliari }\end{array}$ \\
\hline Iperlipidemia familiare combinata & $\begin{array}{l}\text { Simvastatina } \\
\text { Pravastatina } \\
\text { Fluvastatina } \\
\text { Lovastatina } \\
\text { Atorvastatina* }\end{array}$ & $\begin{array}{l}\text { Rosuvastatina } \\
\text { PUFA n-3 } \\
\text { Ezetimibe più statine } \\
\text { (in associazione estemporanea } \\
\text { precostituita)* }\end{array}$ & \\
\hline Disbetalipoproteinemia & $\begin{array}{l}\text { Simvastatina } \\
\text { Pravastatina } \\
\text { Fluvastatina } \\
\text { Lovastatina } \\
\text { Atorvastatina* } \\
\text { Fibrati }\end{array}$ & $\begin{array}{l}\text { Rosuvastatina } \\
\text { Ezetimibe più statine } \\
\text { (in associazione estemporanea } \\
\text { o precostituita)* }\end{array}$ & $\begin{array}{l}\text { Aggiunta di resine sequestranti } \\
\text { gli acidi biliari }\end{array}$ \\
\hline $\begin{array}{l}\text { Iperchilomicronemia e gravi } \\
\text { ipertrigliceridemie }\end{array}$ & $\begin{array}{l}\text { Fibrati } \\
\text { PUFA n-3 }\end{array}$ & Fibrati in associazione a PUFA & \\
\hline
\end{tabular}

*Per i pazienti intolleranti alle statine è rimborsato il trattamento con ezetimibe per conseguire il target terapeutico. PUFA n-3, acidi grassi a lunga catena omega-3.

Circa le indicazioni alla terapia per le dislipidemie familiari, la nota 13 affronta per esteso i criteri classificativi, riassumendo, poi, in una tabella (Tabella 8), i farmaci prescrivibili a carico del SSN secondo diversi livelli di trattamento. ${ }^{23}$

\section{Bibliografia}

1. Downs JR, O'Malley PG. Management of dyslipidemia for cardiovascular disease risk reduction: synopsis of the 2014 U.S. Department of Veterans Affairs and U.S. Department of Defense Clinical Practice Guideline. Ann Intern Med 2015 [Epub ahead of print].

2. Marks D, Thorogood M, Neil HA. A review on the diagnosis, natural history, and treatment of familial hypercholesterolaemia. Atherosclerosis 2003:168:1-14.

3. Fredrickson DS. An international classification of hyperlipidemias and hyperlipoproteinemias. Ann Intern Med 1971;75:471-2.

4. Brunzell JD. Hypertriglyceridemia. N Engl J Med 2007;357:1009-17.

5. Robinson JG. Management of familial hypercholesterolemia: a review of the recommendations from the national lipid association expert panel on familial hypercholesterolemia. JMPC 2013;19:139-49.

6. Orsó E, Ahrens N, Kilalić D, Schmitz G. Familial hypercholesterolemia and lipoprotein(a) hyperlipidemia as independent and combined cardiovascular risk factors. Atheroscler Suppl 2009;10:74-8.

7. Mata P, Alonso R, Ruíz-Garcia A. Familial combined hyperlipidemia: consensus document. Semergen 2014; 40:374-80.

8. Sisman G, Erzin Y, Hatemi I, et al. Hepatobiliary Pancreat Dis Int 2014;13:209-14.
9. Catapano AL, Reiner Z, De Backer G, et al. The Task Force for the management of dyslipidaemias of the European Society of Cardiology (ESC) and the European Atherosclerosis Society. ESC/EAS Guidelines for the management of dyslipidaemias. Atherosclerosis 2011; 217:3-46.

10. Jellinger PS, Smith DA, Mehta AE, et al. American association of clinical endocrinologists' guidelines for management of dyslipidemia and prevention of atherosclerosis. Endocr Pract 2012;18:1-78.

11. Mori TA, Beilin LI. Long-chain omega-3 fatty acids, blood lipids and cardiovascular risk reduction. Curr Opin Lipidol 2001;12:11-7.

12. Perk J, De Backer G, Gohlke H, et al. European guidelines on cardiovascular disease prevention in clinical practice (version 2012). The Fifth Joint Task Force of the European Society of Cardiology and Other Societies on Cardiovascular Disease Prevention in Clinical Practice (constituted by representatives of nine societies and by invited experts). Eur Heart J 2012;33:1635-701.

13. European Association for Cardiovascular Prevention \& Rehabilitation, Reiner Z, Catapano AL, et al. ESC/EAS guidelines for the management of dyslipidaemias: the Task Force for the management of dyslipidaemias of the European Society of Cardiology (ESC) and the European Atherosclerosis Society (EAS). Eur Heart J 2011; 32:1769-818.

14. Najam O, Ray KK. Familial hypercholesterolemia: a review of the natural history, diagnosis and management. Cardiol Ther 2015;4:25-38.

15. PR Newswire. European Commission approves Amgen's new cholesterol-lowering medication Repatha ${ }^{\mathrm{TM}}$ (evolocumab), the first PCSK9 inhibitor to be approved in the world, for treatment of high cholesterol; July 21, 2015. Disponibile su: http://www.multivu.com/players/ English/7414052-amgen-repatha 
16. Robinson JG. Management of familial hypercholesterolemia: a review of the recommendations from the national lipid association expert panel on familial hypercholesterolemia. JMPC 2013;19:139-49.

17. Orsó E, Ahrens N, Kilalić D, Schmitz G. Familial hypercholesterolemia and lipoprotein(a) hyperlipidemia as independent and combined cardiovascular risk factors. Atheroscler Suppl 2009;105:74-8.

18. Trenti C, Negri E, Iori I, Favali D. Hypertriglyceridemia, a common dyslipidemia of complex definition. Ital $\mathrm{J}$ Med 2009;3:220-6.

19. National Cholesterol Education Program (NCEP) Expert Panel on Detection, Evaluation, Treatment of High Blood Cholesterol in Adults (Adult Treatment Panel III). Third Report of the National Cholesterol Education Pro- gram (NCEP) Expert Panel on Detection, Evaluation, and Treatment of High Blood Cholesterol in Adults (Adult Treatment Panel III) final report. Circulation 2002;106(25):3143-421.

20. Mata P, Alonso R, Ruíz-Garcia A. Familial combined hyperlipidemia: consensus document. Semergen 2014; 40:374-80.

21. Agenzia Italiana del Farmaco (AIFA). Note AIFA; aggiornate al 2013. Disponibile su: http://www.agenziafarmaco.gov.it/it/content/note-aifa

22. Goldberg I. Hypertriglyceridemia: impact and treatment. Endocrinol Metab Clin N Am 2009;38:137-49.

23. Agenzia Italiana del Farmaco (AIFA). Nota 13; aggiornate al 2013. Disponibile su: http://www.agenziafarmaco.gov.it/it/content/nota-13 


\title{
Gli omega-3 nell'obesità e nell'insulino-resistenza
}

\author{
Maria Poggiano \\ Medicina Interna, Ospedale Buonconsiglio Fatebenefratelli, Napoli, Italia
}

Il tasso di obesità è in continua crescita globale; di qui la continua necessità di identificare nuove strategie terapeutiche volte a ridurre la morbilità e la mortalità ad essa associate nonché alla riduzione dell'impatto che questa condizione ha sulla spesa sanitaria mondiale.

Un promettente approccio sviluppato negli ultimi anni è l'inclusione nella dieta alimentare dei cosiddetti cibi funzionali (functional foods). Sono sempre più le prove scientifiche a sostegno delle ipotesi che alcuni alimenti e componenti alimentari abbiano effetti benefici che vanno al di là dell'apporto dei nutrienti fondamentali. La ricerca è spesso oggi incentrata sull'identificazione di componenti alimentari biologicamente attivi potenzialmente in grado di ottimizzare il benessere fisico e diminuire il rischio di alcune patologie.

Per esempio un regime alimentare ricco in acidi grassi omega-3 (PUFA n-3) è stato a lungo oggetto di discussione per i benefici che indurrebbe nel management dell'obesità e delle associate alterazioni metaboliche. ${ }^{1}$

Uno studio condotto da Hainault et al., ha recentemente dimostrato che i depositi di tessuto adiposo nei ratti alimentati con cibi addizionati con PUFA n-3 risultavano essere inferiori del 20 e $30 \%$ (tessuto adiposo sottocutaneo e viscerale, rispettivamente) in confronto ad animali a dieta equi-calorica ma non arricchita in omega- $3 .^{2}$

Un ulteriore indagine del gruppo di Belzung et al. suggerisce che esiste un effetto protettivo dose-dipendente di queste molecole come dimostrato da una significativa diminuzione dose dipendente del grasso retroperitoneale. ${ }^{3}$

Corrispondente: Maria Poggiano, Medicina Interna, Ospedale Buonconsiglio Fatebenefratelli, via Manzoni 220, 80123 Napoli, Italia.

E-mail: marypoggiano@gmail.com

Articolo pubblicato secondo la Creative Commons Attribution NonCommercial 3.0 License (CC BY-NC 3.0).

(C) Copyright M. Poggiano, 2015

Licensee PAGEPress, Italy

QUADERNI - Italian Journal of Medicine 2015; 3:260-261
È stato inoltre proposto che l'effetto protettivo sia dovuto ad una riduzione degli accumuli di tessuto adiposo negli adipociti pre-esistenti piuttosto che ad una diminuzione del loro numero complessivo agendo quindi i PUFA n-3 sull'ipertrofia piuttosto che sulla iperplasia adipocitaria. ${ }^{4}$

I PUFA n-3 vengono incorporati negli epatociti e negli adipociti stessi determinando probabilmente una modulazione dell'espressione genica e dell'attività metabolica in questi tessuti. ${ }^{5}$

È noto che l'obesità e le condizioni cliniche ad essa associata (malattie cardiovascolari, diabete, neoplasie) sono fortemente correlate ad uno stato pro-infiammatorio cronico.

I PUFA n-3 attraverso azioni recettore-mediate sembrerebbero agire proprio come potenti anti-infiammatori.

Il GPR120, appartenente alla famiglia dei recettori accoppiati alle G-proteine è stato recentemente identificato tra i possibili 5 recettori stimolati specificamente dai PUFA n-3.

Oh et al., utilizzando gli agonisti sintetici degli acidi grassi eicosapentaenoico docosaesaenoico, hanno valutato gli effetti di queste molecole su cellule di topi obesi esprimenti il recettore GPR120 e in topi GPR120 knock-out. Quando esposti al mediatore, nei topi wild type si osservava una riduzione dei livelli di citochine pro-infiammatorie, quali TNF $\alpha$ e TLR-4, con una conseguente inibizione dei pathways pro-infiammatori, mentre questi eventi non si osservavano nei topi GPR120 knock-out. $^{6}$

Da qui, attraverso una serie di studi del medesimo gruppo è stato proposto che il meccanismo attraverso cui i PUFA n-3 agiscono come agenti anti infiammatori sia legato alla produzione della arrestina 2 , una proteina stimolata dal legame del GPR120 al suo ligando. Secondo lo stesso Oh, la presenza del recettore GPR120 è maggiormente rappresentata nel tessuto adiposo e nei macrofagi CD11+ pro-infiammatori potendo dunque spiegare l'influenza che i PUFA n-3 eserciterebbero su questo tessuto nel mediare la risposta infiammatoria riducendo l'adiposità e l'insulinoresistenza strettamente correlata.

Purtoppo, analogamente a quanto accade per gli studi condotti sulla associazione tra diabete mellito e assunzione di PUFA n-3, anche nell'ambito dell'obe- 
sità e insulino-resistenza, i promettenti risultati emersi dalla sperimentazione animale sull'utilizzo di queste molecole non sono stati confermati sufficientemente in lavori sull'uomo.

Alcuni sudi osservazionali hanno comunque individuato una correlazione positiva tra l'elevato consumo di PUFA n-3 e più bassi livelli di indice di massa corporea. ${ }^{7}$ Questi dati sono stati supportati da numerosi trials che hanno confermato che l'incorporazione cellulare di queste molecole riduce l'adiposità tissutale ${ }^{8}$ regola il senso di sazietà post prandiale e promuove l'incremento della massa magra muscolare. ${ }^{9}$

Per ciò che concerne specificamente l'insulino-resistenza, è stato identificato in modelli animali che $i$ PUFA n-3 esercitano un'azione protettiva modulando direttamente il segnale dell'insulina a livello del tessuto muscolare e adiposo (attraverso l'attività delle PI3-chinasi e GLUT 4).

Nel fegato invece contrasterebbero il processo di steatosi attraverso l'inibizione enzimatica della lipogenesi e promuoverebbero l'ossidazione degli acidi grassi attraverso l'attivazione dei PPAR $\alpha .^{10}$

Numerosi meccanismi molecolari sono tutt'oggi in corso allo scopo di confermare la relazione positiva tra assunzione di PUFA n-3 e benefici metabolici in soggetti che li assumono. Comprendere i meccanismi che legano queste molecole con la patogenesi dell'obesità, e la riduzione della conseguente infiammazione rimane un punto cruciale.

In ogni caso, numerosi studi animali e alcuni studi sull'uomo suggeriscono che l'assunzione di queste sostanze è sicura e apporta benefici sia nel prevenire il guadagno di peso corporeo che nel contrastare le possibili conseguenze dell'insulino-resistenza.

Studi randomizzati e controllati su larga scala sono comunque ancora necessari per confermare i benefici dell'utilizzo di queste sostanze nella terapia dell'obesità e delle alterazioni metaboliche ad essa correlate.

\section{Bibliografia}

1. Campbell SC, Bello NT. Omega-3 fatty acids and obesity. J Food Nutr Disord 2012;1:2.

2. Hainault I, Carolotti M, Hajduch E, et al. Fish oil in a high lard diet prevents obesity, hyperlipemia, and adipocyte insulin resistance in rats. Ann N Y Acad Sci 1993;683:98-101.

3. Belzung F, Raclot T, Groscolas R. Fish oil n-3 fatty acids selectively limit the hypertrophy of abdominal fats depots in growing rats fed high-fat diets. Am J Physiol 1993; 264:1111-8.

4. Parrish CC, Pathy DA, Angel A. Dietetary fish oils limit adipose tissue hypertrophy in rats. Metabolism 1990; 39:217-9.

5. Cunnane SC, McAdoo KR, Horrobin DF. N-3 essential fatty acids decrease weight gain in genetically obese mice. Br J Nutr 1986;56:87-95.

6. Oh DY, Talukdar S, Bae EJ, et al. GPR 120 is an omega3 fatty acids receptor mediating potent anti-infiammatory and insulin-sensitizing effects. Cell 2010;142: 687-98.

7. Cade JE, Burley VJ, Greenwood DC, et al. Women's Cohort study Steering Group. The UK women's cohort study: comparison of vegetarians, fish-eaters and meateaters. Public Health Nutr 2004;7:871-8.

8. Thorsdottir I, Tomasson H, Gunnarsdottir I, et al. Randomized trial of weight-loss-diets for young adults varying in fish and fish oil content. Int $\mathrm{J}$ Obese Lond 2007;31:1560-6

9. Parra D, Ramel A, Bandarra N, et al. A diet rich in long chain omega-3 fatty acids modulates satiety in overweight and obese volunteers during weight loss. Appetite 2008;51:676-80.

10. Delarue J, Lefoll C, Corporeau C, et al. N-3 long chain polyunsaturated fatty acids: a nutritional tool to prevent insulin resistance associated to type 2 diabetes and obesity? Reprod Nutr Dev 2004;44:289-99. 


\title{
Gli omega-3 nel diabete mellito di tipo 2
}

\author{
Maria Poggiano \\ Medicina Interna, Ospedale Buonconsiglio Fatebenefratelli, Napoli, Italia
}

Il diabete mellito, in particolar modo il diabete mellito di tipo 2 (DM 2) sta subendo negli ultimi anni un notevole incremento dell'incidenza e della prevalenza, sia nei paesi industrializzati che in quelli in via di sviluppo.

Essendo uno dei principali fattori di rischio per coronaropatia, accidenti cerebrovascolari, insufficienza renale, retinopatia ed arteropatia periferica, il DM 2 costituisce ad oggi una vera e propria emergenza sanitaria.

Il DM 2 è infatti una delle patologie croniche a più ampia diffusione nel mondo e rappresenta sicuramente una delle più importanti malattie sociali della nostra epoca anche per il progressivo spostamento dell'insorgenza verso fasce di età più giovanili.

Dati recenti dell'International Diabetes Foundation (IDF) segnalano che se nel 2010 oltre 284 milioni circa di persone risultavano affette da DM, nel 2030 i diabetici adulti saranno all'incirca 438 milioni.

È ormai noto che la patogenesi della malattia riconosce due principali fattori di rischio: la dieta e le alterazioni dello stile di vita; è dunque di particolare interesse l'individuazione di alimenti e/o micronutrienti che possano influire sull'insorgenza e il decorso della malattia stessa.

L'associazione tra DM 2 e acidi grassi omega-3 a lunga catena (PUFA n-3) è tuttora oggetto di discussione. Il potenziale ruolo benefico dei PUFA n-3 sulla riduzione del rischio cardiovascolare venne in effetti dall'osservazione della popolazione eschimese della Groenlandia, la quale, nonostante ingerisse più del $40 \%$ delle calorie giornaliere sotto forma di grassi (prevalentemente di origine marina), mostrava una più

Corrispondente: Maria Poggiano, Medicina Interna, Ospedale Buonconsiglio Fatebenefratelli, via Manzoni 220, 80123 Napoli, Italia.

E-mail: marypoggiano@gmail.com

Articolo pubblicato secondo la Creative Commons Attribution NonCommercial 3.0 License (CC BY-NC 3.0).

CCopyright M. Poggiano, 2015

Licensee PAGEPress, Italy

QUADERNI - Italian Journal of Medicine 2015; 3:262-264 bassa incidenza di eventi coronarici acuti rispetto ad individui a dieta normolipidica. ${ }^{1}$

Questi dati sono stati successivamente rafforzati da studi sperimentali successivi che hanno confermato la correlazione positiva tra prevenzione cardiovascolare e assunzione di PUFA n-3 dei quali il pesce che vive abitualmente nei mari freddi è particolarmente ricco.

I principali PUFA n-3 sono l'acido eicosopentanoico (EPA) e l'acido docosopentanoico (DHA), entrambi derivati dall'acido $\alpha$-linoleico (ALA) e inoltre presenti nei pesci grassi come sardine, sgombri, tonno, etc. (Figura 1).

La biosintesi endogena degli acidi grassi a lunga catena ha luogo principalmente a livello epatico e cerebrale attraverso reazioni di elongazione e di desaturazione.

L'ALA, come l'acido linoleico precursore degli omega-6, è definito essenziale, in quanto non è sintetizzato dall'uomo e deve pertanto essere assunto necessariamente con la dieta.

Gli studi più antichi volti a valutare un potenziale effetto benefico dei PUFA n-3 sul profilo lipidico avevano destato preoccupazione perché l'utilizzo di queste molecole sembrava determinare un peggioramento del controllo glicemico.

Studi successivi sintetizzati in meta-analisi hanno consentito una più precisa valutazione del problema.

Nella meta analisi di Friederberg sull'utilizzo dei PUFA n-3 nei pazienti diabetici ${ }^{2}$ è stata evidenziata solo una lieve e non significativa iperglicemia a digiuno mentre la successiva review di Montori et al. ha evidenziato che la supplementazione dietetica con PUFA n-3 determina una riduzione significativa dell'ipertrigliceridemia diabetica senza sensibili variazioni della glicemia. ${ }^{3}$

Dati provenienti da studi animali mostrano che $\mathrm{i}$ PUFA n-3 riducono fortemente l'insulino-resistenza migliorando la tolleranza glicemica specialmente in modelli murini di sindrome metabolica e DM 2. È noto inoltre che la deprivazione di ALA nei ratti aumenta sensibilmente il deposito di massa grassa, la concentrazione plasmatica di glucosio ed insulina e gli indici di insulino-resistenza. ${ }^{4}$

In un importante studio di diversi anni fa, nei ratti alimentati con un'elevata percentuale di lipidi nella 


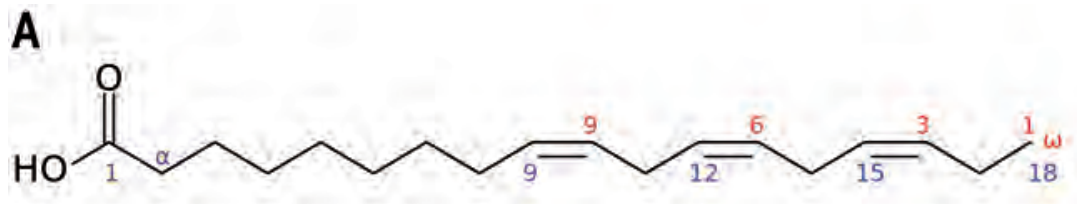

B
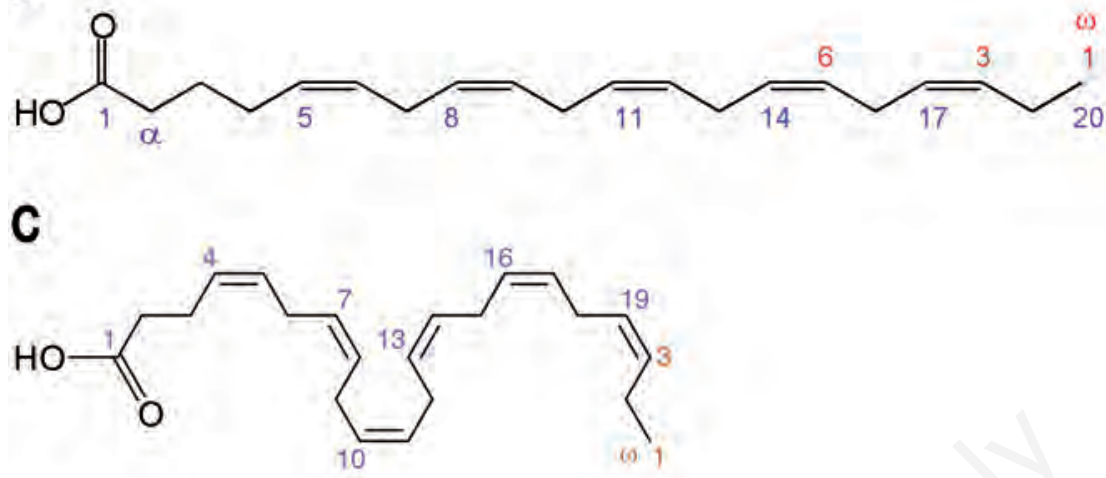

Figura 1. A) Struttura chimica dall'acido $\alpha$-linoleico; B) struttura chimica dell'acido eicosopentanoico; C) struttura chimica di dell'acido docosopentanoico.

dieta (olio di cartamo 59\%), la sostituzione di appena il 6\% di acido linoleico dall'olio di cartamo con PUFA n-3 migliorava sensibilmente l'insulino-resistenza, in particolar modo a livello epatico e muscolare. Inoltre, nello stesso studio, veniva evidenziato un più alto tasso di glicolisi e di glicogenesi nei ratti così alimentati. ${ }^{5,7}$

Un altro interessante studio di Kuda et al., riportava che topi alimentati con una dieta ad alto contenuto lipidico (olio di mais al 35\%) arricchita con DHA/EPA + rosigltazone $(10 \mathrm{mg} / \mathrm{kg})$ si osservavano effetti additivi nella prevenzione di obesità, ipertrofia adipocitaria, dislipidemia, infiammazione del tessuto adiposo viscerale ed insulino-resistenza attraverso incremento delle concentrazioni sieriche di adiponectina. ${ }^{8}$

Purtroppo i promettenti risultati emersi dalla sperimentazione animale spesso non sono stati confermati da studi sull'uomo.

Alcuni autori hanno suggerito che questo dato possa essere attribuibile all'associazione inversa tra insulino-resistenza e attività dell'enzima 5-desaturasi il che potrebbe spiegare la ridotta biodisponibilità dei PUFA n-3 nei pazienti obesi. ${ }^{9}$

Altri autori attribuiscono i diversi risultati sull'azione protettiva degli acidi grassi in studi animali e studi sull'uomo ai contaminanti ambientali contenuti nei pesci, quali il metilmercurio che è stato associato ad insulino-resistenza e che potrebbe dunque attenuare i benefici dell'esposizione a queste molecole.

Recentemente però sono stati pubblicati i risultati di uno studio prospettico che ha analizzato in oltre 19 anni di follow up l'associazione di PUFA n-3 e sviluppo di malattia diabetica in una popolazione finlan- dese costituita da circa 2000 pazienti in una fascia di età compresa tra i 40 e 60 anni. $^{10}$

In questo studio, il lungo follow up ha evidenziato una correlazione negativa tra DM 2 e assunzione di pesce grasso come dimostrato da una più bassa incidenza di malattia diabetica in pazienti con elevate concentrazioni sieriche di acidi grassi a lunga catena.

Lo stesso studio ha indagato l'associazione tra esposizione al mercurio nei capelli dei soggetti inclusi nello studio, quale marker di contaminazione del pesce nella dieta, rivelando che lo stesso mercurio non risultava significativamente correlato allo sviluppo di DM 2 e che non vi fosse interferenza di questo elemento sull'associazione tra livelli di PUFA n-3 e sviluppo di DM 2.

Nella valutazione dei benefici dell'assunzione dei PUFA n-3 in questa particolare categoria di pazienti vanno inoltre considerati i meccanismi indiretti che influiscono sul decorso e la prognosi della malattia diabetica.

Il primo effetto biologico individuato nello studio dei benefici associati all'assunzione di queste molecole, è stato quello ipotrigliceridemizzante; da qui emerge un ruolo rilevante nella gestione della dislipidemia diabetica.

In aggiunta, si è visto come gli oli di pesce diminuiscano l'attivazione delle cellule endoteliali migliorando la disfunzione endoteliale nei soggetti diabetici.

Altri potenziali effetti benefici degli acidi grassi omega-3 a catena lunga sul diabete includono una riduzione dell'aggregazione piastrinica ed effetti antiaritmici. Inoltre, un maggiore consumo di pesce è stato 
correlato ad un più basso rischio di microalbuminuria nei pazienti con diabete di tipo 1 .

Le recenti linee guida della European Society of Cardiology e della European Association for the study of Diabetes raccomandano d'altronde l'assunzione di 2-3 porzioni di pesce ogni settimana, corrispondenti approssimativamente a $0,5 \mathrm{~g}$ di EPA + DHA in pazienti a rischio di sviluppare malattia diabetica o cardiovascolare. ${ }^{11}$

Concludendo, rimane da sottolineare che pur essendo necessarie ulteriori ricerche mediche e biomarker oggettivi di esposizione ai PUFA n-3 per estrapolare informazioni certe sul ruolo che queste molecole rivestono nella fisiopatologia stessa della malattia diabetica, resta chiaro che l'utilizzo dei PUFA n-3 esercita un ruolo favorevole e spesso determinante nelle alterazioni correlate al diabete stesso quali l'infiammazione, l'adiposità e la dislipidemia diabetica e che andrebbe in ogni caso valutata positivamente l'assunzione dietetica di queste sostanze in pazienti a rischio di malattia metabolica.

\section{Bibliografia}

1. Bang HO, Dyerberg J. Plasma lipids and lipoproteins in Greenlanding West-coast Eskimos. Acta Med Scand 1972;192:85.

2. Friedberg CE, Janssen MJFM, Heyne RJ, et al. Fish oil and glycemic control in diabetes: a meta-analysis. Diabetes Care 1998;21:494-500.

3. Montori VM, Former A, Wollan PC, et al. Fish oil supplementation in type 2 diabetes: a quantitative systematic review. Diabetes Care 2000;23:1407-15.
4. Sener A, Zhang Y, Bulur N, et al. The metabolic syndrome of omega-3 depleted rats II. Body weight, adipose tissue mass and glycemic homeostatasis. Int J Mol Med 2009;24:125-9.

5. Poudyal H, Panchal SK, Diwan V, et al. Omega-3 fatty acids and metabolic syndrome: effects and emerging mechanism of action. Progr Lipid Res 2011;50:372-87.

6. Storlien LH, Kraegen EW, Chisholm DJ, et al. Fish oil prevents insulin resistance induced by high fat feeding in rats. Science 1987;237:885-8.

7. Jucker BM, Cline GW, Barucci N, et al. Differential effects of safflower oil versus fish oil feeding on insulinstimulated glycogen synthesis, glycolysis, and pyruvate dehydrogenase flux in skeletal muscle: a 13C nuclear magnetic resonance study. Diabetes 1999;48:134-40.

8. Kuda O, Jelenik T, Jilkova Z et al. N-3 fatty acids and rosiglitazone improve insulin sensitivity trough additive stimulatory effects on muscle glycogen synthesis in mice fed a high-fat diet. Diabetologia 2009;52:941-51.

9. Zhou YE, Kubow S, Dewailly E, et al. Decreased activity of desaturase 5 in association with obesity and insulin resistance aggravates declining long chain n-3 fatty acid status in Cree undergoing dietetary transition. Br J Nutr 2009;102:888-94.

10. Virtanen JK, Mursu J, Voutilainen S, et al. Serum omega-3 polyunsaturated fatty acids and risk of incident type 2 diabetes in men: the Kuopio Ischemic Heart Disease Risk Factor study. Diabetes Care 2014;37:189-96.

11. Rydén L, Grant P, Anke SD, et al. ESC Guidelines on diabetes, pre-diabetes, and cardiovascular diseases developed in collaboration with the EASD: the Task Force on diabetes, pre-diabetes, and cardiovascular diseases of the European Society of Cardiology (ESC) and developed in collaboration with the European Association for the Study of Diabetes (EASD). Eur Heart J 2013;34: 3035-87. 
Farmaci nella forma migliore

\title{
Gli effetti degli omega-3 sulle aritmie
}

\author{
Cristina Cenci \\ Medicina Generale, Ospedale San Matteo degli Infermi, Spoleto (PG), Italia
}

\section{Introduzione}

Gli acidi grassi omega-3 sono grassi polinsaturi che comprendono due acidi grassi di origine animale, contenuti in alta percentuale in pesci e molluschi [acido docosaesaenoico (DHA) e acido eicosapentaenoico (EPA)] e un acido grasso di origine vegetale [acido $\alpha$-linolenico (ALA)] contenuto prevalentemente nell'olio di semi di lino, di soia, di noci e di colza.

L'interesse per l'uso terapeutico degli acidi grassi omega-3 di origine animale (DHA e EPA) nella prevenzione primaria e secondaria delle patologie cardiovascolari, risale ad una pubblicazione del $1976^{1}$ che riporta come l'elevato consumo di olio di pesce tra le popolazioni Inuit della Groenlandia, fosse associata ad una riduzione del rischio di patologia cardiovascolare.

Successivamente studi osservazionali hanno confermato una minore mortalità per eventi cardiovascolari nei paesi a più alto consumo di prodotti ittici come il Giappone. ${ }^{2}$

Si deve però ai risultati dello studio del Gruppo Italiano per lo Studio della Sopravvivenza nell'Infarto Miocardico (GISSI)-prevenzione ${ }^{3}$ la formulazione dell'ipotesi, ancora oggetto di ampio dibattito medicoscientifico, che gli acidi grassi omega-3 possano avere un effetto protettivo sugli eventi aritmici, che cercheremo di sviscerare in questo capitolo.

L'impiego terapeutico di farmaci ad azione non propriamente antiaritmica inoltre, si è fatto strada negli ultimi anni a causa dei risultati non entusiasmanti ottenuti con i farmaci antiaritmici classici, postulando che fosse possibile correggere il substrato alla base dell'aritmia modificandone l'evoluzione e

Corrispondente: Cristina Cenci, Medicina Generale, Ospedale San Matteo degli Infermi, via Loreto, 06049 Spoleto (PG), Italia.

E-mail: Cristina.cenci@uslumbria2.it

Articolo pubblicato secondo la Creative Commons Attribution NonCommercial 3.0 License (CC BY-NC 3.0).

(C) Copyright C. Cenci, 2015

Licensee PAGEPress, Italy

QUADERNI - Italian Journal of Medicine 2015; 3:265-268 prevenendone la prima insorgenza e/o la recidiva (trattamento upstream).

In questo senso si inseriscono gli acidi grassi omega-3 nella eventuale prevenzione primaria e secondaria delle aritmie, possedendo azione antinfiammatoria, antiossidante, di miglioramento della funzionalità endoteliale, ${ }^{4}$ di riduzione della formazione di collagene a livello atriale e di riduzione della vulnerabilità atriale e della durata degli episodi di fibrillazione atriale (FA). ${ }^{5,6}$

\section{Acidi grassi omega-3 e morte cardiaca improvvisa/tachiaritmie ventricolari}

Nello studio GISSI-prevenzione, 11.324 pazienti provenienti da 130 reparti di cardiologia e 42 centri di riabilitazione in Italia, sono stati randomizzati a ricevere una combinazione di EPA/DHA $(850 \mathrm{mg} / 882 \mathrm{mg}$ die, rispettivamente) o placebo (vitamina E) dopo recente infarto acuto del miocardio (entro tre mesi dall'arruolamento) per verificarne l'efficacia nella prevenzione secondaria di eventi cardiovascolari.

Il $93 \%$ dei pazienti inclusi nello studio era in terapia anti aggregante, il 47\% con ACE inibitori, il 44\% con $\beta$-bloccanti, il $5 \%$ con ipolipemizzante all'inizio dello studio e il $46 \%$ con ipolipemizzanti al termine dello studio.

Lo studio GISSI-prevenzione ha dimostrato una riduzione significativa nella mortalità per tutte le cause e nella mortalità per cause cardiovascolari nei 3,5 anni di follow-up, effetto già evidente nei primi 3-4 mesi.

Le conclusioni degli autori furono che questo risultato positivo potesse derivare dalla riduzione delle morti improvvise e pertanto gli stessi aprirono la strada all'ipotesi che gli omega-3 potessero avere un potenziale effetto antiaritmico che proteggesse dalla morte cardiaca improvvisa.

Un trial clinico controllato e randomizzato con limiti metodologici per la mancanza di cecità e per il tipo di intervento proposto, ${ }^{7}$ ha invece evidenziato un aumento di morti improvvise dell' $1,6 \%$ a 9 anni, in un gruppo di 3114 pazienti anginosi ai quali veniva consigliato di assumere 2 porzioni di pesce grasso a settimana in media o analoga quantità di EPA in capsule.

Tre studi clinici controllati randomizzati (RCTs) ${ }^{8-10}$ e una metanalisi ${ }^{11}$ successivi hanno testato l'ipotesi degli 
effetti protettivi degli acidi grassi omega-3 nella prevenzione della morte cardiaca improvvisa in $\mathrm{pz}$ con defibrillatore cardiaco impiantabile, ma nessuno di essi ha dimostrato un effetto benefico sugli outcome considerati.

Proprio per l'importanza clinica e di salute pubblica della prevenzione della morte cardiaca improvvisa in assenza di chiare evidenze che gli omega-3 possano realmente esercitare su di essa un ruolo protettivo, un'ulteriore metanalisi del 2009 pubblicata sul BMJ ha voluto verificare gli effetti degli omega-3 sulla morte improvvisa e su qualsiasi evento aritmico registrato all'elettrocardiogramma (outcome primari), in pazienti con defibrillatore elettrico impiantabile. ${ }^{12}$

Nella revisione sono stati inclusi 12 RCTs di cui 2 studi di più grandi dimensioni hanno fornito la maggior parte dei pazienti (29.979/32.779 totali).

Quali outcome secondari lo studio ha valutato la mortalità per qualsiasi causa e la mortalità per cause cardiache.

Dalla revisione sistematica è emerso come la supplementazione con olio di pesce non fosse associata ad una significativa riduzione del rischio di eventi aritmici o di morte cardiaca improvvisa nei pazienti con defibrillatore elettrico impiantabile, né ad una riduzione della mortalità per tutte le cause.

È stata invece riscontrata una riduzione statisticamente significativa (20 per cento) del rischio di morte per cause cardiache ( $11 \mathrm{RCT}, \mathrm{n}=32.519$ ) odds ratio 0,80 (95\% intervallo di confidenza: $0,69,0,92, \mathrm{P}=0,002$ ).

Una successiva metanalisi del $2013^{13}$ ha confermato l'assenza di effetto protettivo degli omega-3 sul rischio di morte improvvisa o di aritmia ventricolare.

Nell'interpretazione di questi risultati va sottolineato come i pazienti con defibrillatore elettrico impiantabile siano una minoranza di pazienti rispetto alla popolazione generale affetta da patologia cardiovascolare e come l'eziologia delle tachiaritmie ventricolari nella stessa popolazione di pazienti, sia differente dalla eziologia della fibrillazione ventricolare di prima insorgenza che è causa di morte cardiovascolare e morte improvvisa nella popolazione generale e che sottende di solito una genesi ischemica.

\section{Acidi grassi omega-3 e fibrillazione atriale}

Anche per quanto riguarda il ruolo protettivo degli acidi grassi omega-3 nei confronti della prevenzione della recidiva di FA dopo cardioversione o dopo cardiochirurgia, la maggior parte degli studi clinici sono stati realizzati partendo dalle conclusioni dello studio GISSI-prevenzione e da evidenze derivanti da studi sperimentali in vivo ed in vitro e da studi epidemiologici che per completezza di informazioni analizzeremo nel capitolo successivo per mostrare quali siano i meccanizmi d'azione ipotizzati, per giustificare un eventuale e potenziale ruolo degli omega-3 sul ritmo cardiaco.

Gli studi clinici non sperimentali hanno dimostrato conclusioni discordanti circa l'effetto degli ac. grassi omega-3 nella FA, dal momento che alcuni di essi hanno evidenziato un'associazione favorevole tra consumo di acidi grassi polinsaturi omega-3 e riduzione del rischio di FA, ${ }^{14,15}$ mentre altri hanno invece fallito nel dimostrare tale associazione. ${ }^{16,17}$

Un recente RCTs in doppio cieco ha incluso 1516 pazienti sottoposti a cardiochirurgia e provenienti da 28 centri negli USA, Italia e Argentina, randomizzati ad assumere acidi grassi omega-3 o placebo, dimostrando nessuna riduzione statisticamente significativa, del rischio di FA post operatoria nel gruppo trattato con omega-3. ${ }^{18}$

Anche due successive metanalisi non hanno dimostrato alcun ruolo degli acidi grassi omega-3 nella riduzione dell'incidenza di FA dopo un primo episodio o dopo cardiochirurgia. ${ }^{19,20}$

Nel complesso, questi studi forniscono prove di alta qualità, che a breve termine gli omega-3 non riducono il rischio di FA post-operatoria e che non sembrano ridurre il rischio di FA ricorrente nei pazienti con fibrillazione atriale preesistente, almeno nel medio termine (sei mesi ad un anno).

Le stesse conclusioni sono assunte dalle linee guida $^{21}$ dell'Associazione Italiana di Aritmologia Clinica (AIAC) aggiornate al 2013, che concludono per l'assenza di dati sufficienti per proporre la terapia con omega-3 nella prevenzione primaria e secondaria della FA (raccomandazione di classe II b livello evidenza A).

\section{Possibili meccanismi d'azione degli acidi grassi omega-3}

Numerosi meccanismi d'azione sono stati proposti e studiati per spiegare i potenziali effetti antiaritmici degli acidi grassi polinsaturi omega-3 a livello cardiaco, ma nessuno di essi è stato finora validato.

L'ipotesi a tutt'oggi più accreditata si basa su modelli animali in cui viene loro attribuito un effetto protettivo qualora l'aritmia avvenga a seguito di un episodio ischemico acuto.

L'esatta sequenza di azioni attraverso cui ciò si realizzi non è nota ma sembrerebbe coinvolgere 4 seguenti effetti.

\section{Effetto diretto sulla membrana plasmatica}

Un incremento del $10 \%$ con la dieta, di acidi grassi omega-3, può duplicare il contenuto di DHA nella membrana plasmatica miocardica dopo $2 \mathrm{gg}$, mentre l'accumulo in essa di EPA è più basso. ${ }^{22}$ Questa modifica nella composizione dei fosfolipidi di membrana, potrebbe comportare profondi effetti nello scambio io- 
nico tra i diversi compartimenti cellulari rendola meno suscettibile allo stimolo antiaritmico dopo ischemia cardiaca.

\section{Effetto diretto sui canali ionici}

Le evidenze disponibili in letteratura mostrano in modelli animali, come gli omega-3 possano interferire con i canali cellulari del sodio, ${ }^{23,24}$ del potassio ${ }^{25,26} \mathrm{e}$ del calcio ${ }^{27,28}$ a concentrazioni plasmatiche analoghe a quelle che si ritrovano nel plasma di soggetti umani studiati nei trial clinici precedentemente descritti. L'effetto complessivo sarebbe una significativa inibizione dose dipendente di tali canali che comporterebbe un effetto protettivo verso la morte cardiaca improvvisa e le aritmie. ${ }^{29,30}$

\section{Effetto sui mediatori dell'infiammazione}

Studi clinici ${ }^{31}$ hanno dimostrato come i metaboliti derivati dagli acidi grassi omega-6 (acido linoleico e acido arachidonico), tra cui il trombossano A2 e la prostaglandina F2 $\alpha$ siano implicati nella genesi di tachicardie associata a inflammatione sistemica. La supplementazione dietetica o farmacologica con acidi grassi omega-3 sembrerebbe essere efficace per modificare la composizione in acidi grassi, della membrana plasmatica dei miociti cardiaci, riducendo in questo modo la produzione di trombossano e di altri mediatori proinfiammatori, che esercitano effetto proaritmico. Infatti successivamente ad un danno ischemico, la lipolisi cardiaca comporterebbe la liberazione nel plasma di acidi grassi liberi non più legati alla membrana plasmatica fosfolipidica e l'attivazione della fosfolipasi A2 e di metaboliti dell'acido arachidonico ${ }^{32}$ che sarebbero contrastati nella loro azione dai metaboliti degli acidi grassi omega-3 che attivano invece la fosfolipasi A3 che genera metaboliti antiaritmici.

\section{Regolazione dell'attività delle proteine chinasi}

In modelli animali la supplementazione con acidi grassi omega-3 può ridurre la responsività dei recettori $\beta$-adrenergici e inibire l'attività delle proteine chinasi ${ }^{33}$ che svolgono un ruolo attivo nella modulazione dei canali ionici e sono correlati alla morte improvvisa negli uomini. ${ }^{34}$

\section{Messaggi chiave}

- Allo stato attuale delle conoscenze, non è possibile definire con certezza quali che siano i potenziali effetti degli acidi grassi omega-3 sulla morte cardiaca improvvisa nella popolazione generale di soggetti sani con patologia cardiovascolare.

- Sono ancora necessari ulteriori studi per chiarire i potenziali effetti dell'assunzione con la dieta o della supplementare a lungo termine con acidi grassi omega-3, nei pazienti con FA di nuova insorgenza.

- Diversi sono i meccaniami d'azione ipotizzati per spiegare il potenziale effetto benefico degli acidi grassi omega-3 sulle aritmie ma nessuno di essi è ancora stato validato.

\section{Bibliografia}

1. Bang HO, Dyerberg J, Hjoorne N. The composition of food consumed by Greenland eskimos. Acta Med Scand 1976;200:69-73.

2. Kris-Etherton PM, Harris WS, Appel LJ; American Heart Association. Nutrition Committee. Fish consumption, fish oil, omega-3 fatty acids and cardiovascular disease. Circulation 2002;106:2747-57.

3. [No authors listed]. Dietary supplementation with n-3 polyunsaturated fatty acids and vitamin $\mathrm{E}$ after myocardial infarction: results of the GISSI-prevenzione trial. Gruppo Italiano per lo Studio della Sopravvivenza nel1'Infarto Miocardico. Lancet 1999;354:447-55.

4. Jahangiri A, Leifert WR, Patten GS, Mc-Murchie EJ. Termination of asynchronous contractile activity in rat atrial myocytes by n-3 polyunsaturated fatty acids. Mol Cell Biochem 2000;206:33-41.

5. Ninio DM, Murphy KJ, Howe PR, Saint DA. Dietary fish oil protects against stretch-induced vulnerability to atrial fibrillation in a rabbit model. J Cardiovasc Electrophysiol 2005;16:1189-94

6. Sakabe M, Shiroshita-Takeshita A, Maguy A, et al. Omega-3 polyunsaturated fatty acids prevent atrial fibrillation with heart failure but not atrial tachycardia remodeling. Circulation 2007;116:2101-9.

7. Burr ML, Ashfield-Watt PA, Dunstan FD, et al. Lack of benefit of dietary advice to men with angina: results of a controlled trial. Eur J Clin Nutr 2003;57:193-200.

8. Raitt MH, Connor WE, Morris C, et al. Fish oil supplementation and risk of ventricular tachycardia and ventricular fibrillation in patients with implantable defibrillators: a randomized controlled trial. JAMA 2005;293:2884-91.

9. Leaf A, Albert CM, Josephson M, et al. Prevention of fatal arrhythmias in high-risk subjects by fish oil n-3 fatty acid intake. Circulation 2005;112:2762-8.

10. Brouwer et al. Effect of fish oil on ventricular tachyarrhythmia and death in patients with implantable cardioverter defibrillators: the study on omega-3 fatty acids and ventricular arrhythmia (SOFA) randomized trial. JAMA 2006;295:2613-9.

11. Jenkins DJ, Josse AR, Beyene J, et al. Fish oil supplementation in patients with implantable cardioverter defibrillators: a meta-analysis. CMAJ 2008;178:157-64.

12. León H, Shibata MC, Sivakumaran S, et al. Effect on fish oil on arrythmias and mortality: systematic review. BMJ 2008;337:a2931.

13. Khoueiry G, Abi Rafeh N, Sullivan E, et al. Do omega3 polyunsaturated fatty acids reduce risk of sudden cardiac death and ventricular arrhythmias? A meta-analysis of randomized trials. Heart Lung 2013;42:251-6.

14. Mozaffarian D, Psaty BM. Fish intake and risk of incident atrial fibrillation. Circulation 2004;110:368. 
15. Virtanen JK, Mursu J, Voutilainen S, Tuomainen TP, et al. Serum long-chain n-3 polyunsaturated fatty acids and risk of hospital diagnosis of atrial fibrillation in men. Circulation 2009;120:2315.

16. Frost L, Vestergaard P. n-3 Fatty acids consumed from fish and risk of atrial fibrillation or flutter: the Danish diet, cancer, and health study. Am J Clin Nutr 2005; 81:50.

17. Berry JD, Prineas RJ, van Horn L, et al. Dietary fish intake and incident atrial fibrillation (from the Women's Health Initiative). Am J Cardiol 2010;105:844.

18. Mozaffarian D, Marchioli R, Macchia A, et al. Fish oil and postoperative atrial fibrillation: the omega-3 fatty acids for prevention of post-operative atrial fibrillation (OPERA) randomized trial. JAMA 2012;308:2001.

19. Mariani J, Doval HC, Nul D, et al. N-3 polyunsaturated fatty acids to prevent atrial fibrillation: updated systematic review and meta-analysis of randomized controlled trials. J Am Heart Assoc 2013;2:e005033.

20. Nigam A, Talajic M. Fish oil for the reduction of atrial fibrillation recurrence, inflammation, and oxidative stress. J Am Coll Cardiol 2014;64:1441.

21. Raviele A, Disertori M, Alboni P, et al. Linee guida AIAC per la gestione e il trattamento della fibrillazione atriale. Aggiornamento 2013. G Ital Cardiol 2013;14: 215-40.

22. Owen AJ, Peter-Przyborowska BA, Hoy AJ, McLennan PL. Dietary fish oil dose- and time-response effects on cardiac phospholipid fatty acid composition. Lipids 2004;39:955-61.

23. Leifert WR, McMurchie EJ, Saint DA. Inhibition of cardiac sodium currents in adult rat myocytes by $n-3$ polyunsaturated fatty acids. J Physiol 1999;520:671-9.

24. Xiao YF, Kang JX, Morgan JP, Leaf A. Blocking effects of polyunsaturated fatty acids on $\mathrm{Na}+$ channels of neo- natal rat ventricular myocytes. Proc Natl Acad Sci U S A 1995;92:11000-4.

25. McLennan P, Howe P, Abeywardena M, et al. The cardiovascular protective role of docosahexaenoic acid. Eur J Pharmacol 1996;300:83-9.

26. Rouzaire-Dubois B, Gerard V, Dubois JM. Modification of $\mathrm{K}+$ channel properties induced by fatty acids in neuroblastoma cells. Pflugers Arch 1991;419:467-71.

27. Hallaq H, Smith TW, Leaf A. Modulation of dihydropyridine-sensitive calcium channels in heart cells by fish oil fatty acids. Proc Natl Acad Sci U S A 1992;89:1760-4.

28 Rodrigo GC, Dhanapala S, Macknight AD. Effects of eicosapentaenoic acid on the contraction of intact, and spontaneous contraction of chemically permeabilized mammalian ventricular myocytes. J Mol Cell Cardiol 1999;31:733-43.

29. Billman GE, Kang JX, Leaf A. Prevention of sudden cardiac death by dietary pure omega-3 polyunsaturated fatty acids in dogs. Circulation 1999; 99:2452-7.

30. Leaf A, Kang JX, Xiao YF, Billman GE. Clinical prevention of sudden cardiac death by $n-3$ polyunsaturated fatty acids and mechanism of prevention of arrhythmias by n-3 fish oils. Circulation 2003;107:2646-52.

31. Takayama K, Yuhki K, Ono K, et al. Thromboxane A2 and prostaglandin F2alpha mediate inflammatory tachycardia. Nat Med 2005;11:562-6.

32. Henry PD, Pacifico A. Altering molecular mechanisms to prevent sudden arrhythmic death. Lancet 1998;351: 1276-8.

33. Reibel DK, Holahan MA, Hock CE. Effects of dietary fish oil on cardiac responsiveness to adrenoceptor stimulation. Am J Physiol 1988;254:H494-9.

34. Pogwizd SM, Bers DM. Cellular basis of triggered arrhythmias in heart failure. Trends Cardiovasc Med 2004;14:61-6. 
Farmaci nella forma migliore

\title{
Gli effetti degli omega-3 nella prevenzione dell'infarto miocardico
}

\author{
Riccardo Gerloni \\ Medicina Interna, Ospedale Cattinara, Trieste, Italia
}

Si inizia a discutere di acidi grassi a lunga catena omega-3 (PUFA n-3) nel lontano 1980, quando si ipotizza che la dieta degli eschimesi, così ricca di pesce, sia responsabile della loro molto bassa morbilità cardiovascolare. ${ }^{1}$ A seguire numerosissimi studi, dapprima meramente osservazionali, poi sperimentali su animali, fino a veri e propri trial clinici randomizzati, che hanno generato, tuttavia, una serie di dati in parte contradditori tanto che ad oggi l'utilità degli acidi grassi nel ridurre il rischio cardiovascolare non è ancora stata chiarita.

Gli studi osservazionali condotti su popolazioni occidentali che consumavano abitualmente una scarsa quantità di omega-3 non avevano dimostrato un chiaro beneficio sull'incidenza di infarto miocardico. ${ }^{2-4}$ Uno studio prospettico su 41.578 giapponesi $^{5}$ aveva chiarito poi che l'assunzione di PUFA n- 3 tramite dieta doveva essere di una certa quantità e, se superava i 2,1 $\mathrm{g} /$ die, poteva addirittura ridurre l'incidenza di infarto miocardico del $67 \%$. Infine uno studio prospettico osservazionale aveva correlato gli introiti di PUFA n-3, riassunti attraverso un questionario, con l'incidenza di infarto miocardico, dimostrando che solo nei soggetti diabetici vi era un effetto protettivo. ${ }^{6}$

Gli studi clinici randomizzati e controllati, stimolati da questi studi osservazionali, non hanno convincentemente e uniformemente dimostrato una riduzione del rischio di infarto, ma hanno messo in luce piuttosto alcune evidenze di benefici sulla morte cardiovascolare e particolarmente sulla morte improvvisa (Tabella 1). E così lo studio DART (diet and reinfarction trial), ${ }^{7}$ che, randomizzando oltre 2000 persone ad una dieta ricca in PUFA $n-3$, aveva dimostrato una riduzione della mortalità per tutte le cause pari al 29\%. Nel 1999

Corrispondente: Riccardo Gerloni, Medicina Interna, Ospedale Cattinara, strada di Fiume 447, 34149 Trieste, Italia. E-mail: riccardogerloni@libero.it

Articolo pubblicato secondo la Creative Commons Attribution NonCommercial 3.0 License (CC BY-NC 3.0).

C Copyright R. Gerloni, 2015

Licensee PAGEPress, Italy

QUADERNI - Italian Journal of Medicine 2015; 3:269-273 veniva pubblicato lo studio del Gruppo Italiano per lo Studio della Sopravvivenza nell'Infarto Miocardico (GISSI)-prevenzione, ${ }^{8}$ che, arruolando oltre 11.000 pazienti colpiti recentemente da infarto e sottoposti ad una supplementazione con $850 \mathrm{mg} /$ die di acido eicosapentaenoico (EPA) e di docosaesaenoico (DHA), aveva dimostrato una riduzione di mortalità per tutte le cause pari al $20 \%$, di mortalità cardiovascolare pari al $30 \%$ e di morte improvvisa pari al $45 \%$. I dati erano così promettenti che Yokoyama e coll. ${ }^{9}$ nel 2007 arruolavano oltre 18.000 pazienti, non più reduci da infarto ma con ipercolesterolemia, e dimostravano una riduzione del $19 \%$ di eventi coronarici ad un followup di 4,6 anni nei soggetti che alla statina associavano una dose elevata di EPA, pari a $1800 \mathrm{mg} /$ die. Un anno dopo appariva in letteratura lo studio GISSI-HF (heart failure), ${ }^{10}$ che presentava pure risultati positivi anche se meno entusiasmanti, somministrando $850 \mathrm{mg} / \mathrm{die}$ di EPA e DHA a soggetti affetti da scompenso cardiaco. Si assisteva ad una riduzione del 2,3\% di morte o ricovero per cause cardiovascolari.

Altrettanto numerosi sono gli studi pubblicati che non hanno dimostrato alcun beneficio con la somministrazione di PUFA n-3 (Tabella 2). Già nel 2003 Burr e coll., ${ }^{11}$ pubblicando lo studio DART2, simile nel disegno al DART, non avevano dimostrato benefici nei soggetti con angina pectoris. Più recenti gli studi Alpha Omega ed Omega, ${ }^{12,13}$ trials randomizzati su pazienti reduci da infarto, che non trovavano benefici sulla mortalità cardiovascolare, la morte improvvisa e gli eventi coronarici. In realtà una post hoc analisi su soggetti diabetici aveva dimostrato una riduzione di mortalità coronarica e di aritmie ventricolari pari al $40 \% \cdot{ }^{14} \mathrm{Nel} 2012$ veniva pubblicato lo studio ORIGIN (outcome reduction with an initial glargine intervention), coinvolgente oltre 12.000 pazienti con multipli fattori di rischio cardiovascolare, che dimostrava assenza di benefico ad un anno dalla somministrazione di un grammo/die di PUFA n-3. ${ }^{15} \mathrm{Nel} 2014$ lo studio AREDS2 (age-related eye disease study 2) dimostrava in 4203 partecipanti, affetti da maculopatia e cardiopatia ischemica con eventi datati almeno 12 mesi prima, l'assenza di effetto dei PUFA n-3 su infarto miocardico, ictus e morte cardiovascolare. ${ }^{16}$

Per cercare di far chiarezze tra questa nutrita ma discordante letteratura sono comparse recentemente 
alcune metanalisi con tutti i limiti di questo tipo di revisione (non omogenee le popolazioni di riferimento, i dosaggi dei PUFA n-3, la durata dei trials). Una recente metanalisi del 2012 non ha trovato beneficio sulla riduzione di infarto miocardico. ${ }^{17}$ Una metanalisi successiva, ${ }^{18}$ che considerava oltre 16.000 pazienti, ha dimostrato una riduzione della mortalità cardiovascolare, ma non degli eventi coronarici. Infine Enns e coll. ${ }^{19}$ in una metanalisi coinvolgente solo 3 trials e 396 pazienti hanno riscontrato inefficacia dei PUFA n-3 nel ridurre gli eventi cardiovascolari nei soggetti con arteriopatia periferica.

Eppure i presupposti fisiopatologici di efficacia dei PUFA n-3 ci sono tutti: è stato dimostrato, infatti, che 1'utilizzo di omega-3 è in grado di modificare in maniera favorevole i principali fattori di rischio cardiovascolare. Così in una metanalisi di 36 studi clinici randomizzati ${ }^{20}$ la pressione arteriosa sistolica, dopo supplementazione con olio di pesce per almeno 8 settimane, si riduceva di $3,5 \mathrm{mmHg}$ e quella diastolica di 2,4 mmHg considerando i soggetti con età maggiore di 45 anni. Poiché i vari trials inclusi nella metanalisi hanno utilizzato supplementazione a dosaggi diversi si può asserire che l'effetto sulla pressione arteriosa non sembra avere una relazione lineare con la dose. Gli studi osservazionali sull'uomo e quelli sperimen- tali sull'animale hanno evidenziato che l'effetto sulla pressione arteriosa era provocato da una diminuzione delle resistenze vascolari con mantenimento della portata cardiaca immodificata. ${ }^{21,22}$ Probabile che alla base di tutto vi sia un aumento della produzione di ossido nitrico. ${ }^{23-26}$

Anche il profilo lipidico viene modificato in maniera favorevole dalla somministrazione di PUFA n-3 e, accanto alla nota riduzione dei trigliceridi fino al $25-30 \%$ il cui effetto sulla riduzione del rischio cardiovascolare è però alquanto discutibile, ${ }^{27-29}$ si verifica un lieve aumento delle lipoproteine ad alta densità e della concentrazione delle lipoproteine a bassa densità (LDL) con riduzione delle LDL piccole e dense, ritenute queste ultime convincentemente responsabili della formazione e sviluppo della placca ateromasica. ${ }^{30-32}$ Tali modificazioni sul profilo lipidico avvengono per somministrazione di PUFA n-3 ad alto dosaggio, non utilizzato solitamente nei trials formulati per verificare l'effetto di tali sostanze sulla morbilità e mortalità cardiovascolare. Quindi i PUFA n-3 migliorano il profilo lipidico, ma non è tramite questo meccanismo che si può spiegare gli effetti favorevoli verificatesi nei soggetti arruolati nei trials e sottoposti a supplementazione.

Dalla metanalisi di Mozaffarian e coll..$^{33}$ appare

Tabella 1. Studi clinici randomizzati a sostegno di acidi grassi a lunga catena omega-3.

\begin{tabular}{lllll}
\hline Studio & $\begin{array}{l}\text { Caratteristiche dei } \\
\text { partecipanti }\end{array}$ & $\begin{array}{l}\text { Numero di } \\
\text { partecipanti }\end{array}$ & Dose di PUFA n-3 & Risultati \\
\hline DART & Reduci da infarto miocardico & 2033 & $350 \mathrm{mg}$ EPA & Riduzione 29\% della mortalità \\
\hline GISSI-prevenzione & Reduci da infarto miocardico & 11.324 & $850 \mathrm{mg}$ EPA/DHA & $\begin{array}{l}\text { Riduzione: } \\
20 \% \text { eventi cardiovascolari } \\
\end{array}$ \\
& & & $\begin{array}{l}30 \% \text { morte cardiovascolare } \\
45 \% \text { morte aritmica }\end{array}$ \\
\hline JELIS & Ipercolesterolemici & 18.645 & $1800 \mathrm{mg}$ EPA & Riduzione 19\% eventi cardiovascolari \\
\hline GISSI-HF & Affetti da scompenso cardiaco & 6975 & $850 \mathrm{mg}$ EPA/DHA & $\begin{array}{l}\text { Riduzione 6\% ospedalizzazione o morte } \\
\text { cardiovascolare }\end{array}$ \\
\hline
\end{tabular}

PUFA n-3, acidi grassi a lunga catena omega-3; EPA, acido eicosapentaenoico; DHA, acido docosaesaenoico.

Tabella 2. Studi clinici randomizzati contrari all'uso di acidi grassi a lunga catena omega-3.

\begin{tabular}{lllll}
\hline Studio & $\begin{array}{l}\text { Caratteristiche dei } \\
\text { partecipanti }\end{array}$ & $\begin{array}{l}\text { Numero di } \\
\text { partecipanti }\end{array}$ & Dose di PUFA n-3 & Risultati \\
\hline DART-2 & Affetti da angina & 3114 & $430 \mathrm{mg}$ EPA & Aumento morte cardiovascolare \\
\hline Alpha-Omega & Reduci da infarto miocardico & 4837 & $400 \mathrm{mg}$ EPA/DHA & Non beneficio \\
\hline OMEGA & Reduci da infarto miocardico & 3851 & $840 \mathrm{mg}$ EPA/DHA & Non beneficio \\
\hline ORIGIN & Diabetici & 12.536 & $840 \mathrm{mg}$ EPA/DHA & Non beneficio \\
\hline AREDS2 & $\begin{array}{l}\text { Maculopatia ed evento } \\
\text { cardiovascolare datato }\end{array}$ & 4203 & $\begin{array}{l}350 \mathrm{mg} \text { DHA } \\
650 \mathrm{mg} \text { EPA }\end{array}$ & Non beneficio \\
\hline
\end{tabular}

PUFA n-3, acidi grassi a lunga catena omega-3; EPA, acido eicosapentaenoico; DHA, acido docosaesaenoico. 
evidente che l'effetto dei PUFA n-3 si esplica anche sulla frequenza cardiaca con una riduzione pari a 1,6 bpm, raggiungendo i 2,5 bpm nei trials con durata superiore a 12 settimane. Essendo i PUFA n-3 integrati nei fosfolipidi di membrana delle cellule cardiache, è verosimile che la loro azione sulla frequenza cardiaca si esplichi modulando i canali ionici. ${ }^{34,35}$ Va a questo proposito considerata anche l'azione dei PUFA n-3 sul miglioramento della funzione diastolica ventricolare sinistra e sull'aumento del tono vagale. ${ }^{36}$

Per ultimo numerosi sono i lavori che rilevano un effetto dei PUFA n-3 nel modulare l'infiammazione, fattore notoriamente coinvolto nello sviluppo della placca e soprattutto nella sua instabilizzazione. Tale modulazione si osserva solitamente per dosaggi elevati di PUFA n-3 e quindi verosimilmente non è da contemplare tra i meccanismi da chiamare in causa per spiegare i dati favorevoli dei trials clinici. ${ }^{37}$

Come si conciliano allora i buoni presupposti fisiopatologici dei PUFA n-3 con i trials così contrastanti comparsi in letteratura? Molte sono le spiegazioni proposte: ${ }^{38-40}$ innanzitutto la durata del trattamento, così differente tra gli studi osservazionali, che riflettono uno stile di alimentazione proprio del paziente, e i trial clinici che, per quanto lunghi, durano qualche anno e presentano compliance subottimale dei pazienti; inoltre, la differente dose di PUFA n-3 ricevuta dai pazienti nei vari studi (nello studio Alpha Omega $^{12}$ la dose era la metà di quella usata negli studi GISSI $^{8,10}$ ) possono far ipotizzare effetti diversi e, comunque, rendono i dati non paragonabili tra loro e limitano il potere delle metanalisi; ancora, il tipo di PUFA n-3 considerato: seppure nella maggior parte dei casi la supplementazione consisteva in EPA e DHA, la percentuale di questi variava in modo significativo e più di qualche autore considera l'azione benefica di PUFA n-3 a carico principalmente di DHA, perché è quello che viene poi incorporato nei fosfolipidi di membrana delle cellule cardiache ${ }^{41}$ e sembra quindi responsabile degli effetti benefici sulla morte improvvisa. Non va dimenticata inoltre l'osservazione di Superko e coll., ${ }^{42}$ che propongono un effetto non dose dipendente ma livello di plasma dipendente, a dire che la somministrazione di una certa quantità di PUFA n-3 non produce matematicamente un certo livello di sostanza circolante, perché si scontra con una farmacocinetica differente tra gli individui ${ }^{43}$ e perché questi ultimi presentano livelli plasmatici iniziali di PUFA n-3 differenti. Ecco perché si assiste ad un grande effetto nelle popolazioni che non assumono nella loro dieta pesce, e ad uno risicabile, se non proprio nullo, laddove la popolazione in oggetto si nutre in maniera considerevole di pesce. Inoltre $\mathrm{i}$ grandi trials hanno arruolato popolazioni differenti: alcuni consideravano pazienti reduci da infarto, altri pazienti con singoli o multipli fattori di rischio cardiovasco- lare. Alcuni arruolavano pazienti con terapia massimale e ottima compliance come lo studio Alpha Omega, ${ }^{12}$ dove 1' $85 \%$ dei pazienti faceva uso di ipolipemizzanti, il 98\% di anti-trombotici, il 91\% di antiipertensivi. Invece, nello studio GISSI-prevenzione, ${ }^{8}$ che arruolava pazienti reduci da infarto miocardico, solo il $5 \%$ utilizzava ipolipemizzanti e solo il $44 \% \beta$ bloccanti. E allora non è inverosimile pensare che un gruppo di pazienti trattati al meglio della terapia medica possa beneficiare meno o proprio non beneficiare della terapia con PUFA n-3. Infine potrebbe essere importante considerare nei pazienti reduci da infarto il tempo trascorso dall'evento. Un recente studio restrospettivo su oltre 2000 pazienti ${ }^{44}$ ha dimostrato una riduzione della mortalità in soggetti reduci da infarto che iniziavano ad assumere un grammo/die di PUFA n-3 entro 90 giorni dall'evento. Non è inverosimile pensare che tale effetto si ridurrebbe se la somministrazione avvenisse a distanza di tempo maggiore.

Possiamo quindi affermare che il capitolo dei PUFA n-3 nel ridurre il rischio di infarto è ricco di letteratura contrastante, probabilmente determinata da diversità di impostazione dei vari studi su modalità e tempi di somministrazione, popolazione in esame, dosaggio ematico dei PUFA n-3. Sono stati dimostrati al tempo stesso i presupposti fisiopatologici, per cui è verosimile che, trovata la dose corretta, il tipo di PUFA n-3, i tempi di somministrazione indispensabile ad osservare un effetto favorevole e soprattutto la popolazione target, i PUFA n-3 potrebbero incidere significativamente sulla morbilità cardiovascolare $\mathrm{e}$ in particolare sugli eventi coronarici. È necessario quindi attuare ulteriori trials con ampia casistica e lungo follow-up per chiarire a quali pazienti somministrare integrazioni di PUFA n-3. Per ora nei pazienti con alto rischio cardiovascolare una raccomandazione sullo stile di vita comprensivo di adeguato consumo di pesce deve essere messa in opera. ${ }^{38}$ Eventualmente in quelli che sono intolleranti o riluttanti a seguire questa indicazione può esser considerata opportuna l'integrazione con PUFA n-3. Somministrare tale supplementazione ai diabetici, ai reduci da infarto miocardico o ai pazienti con scompenso cardiaco (sottogruppi che più di altri hanno beneficiato della supplementazione di PUFA n-3 nei trials pubblicati) per ridurre l'incidenza di eventi ischemici resta un fatto opinabile. ${ }^{45}$

\section{Messaggi chiave}

- Da una serie di studi prospettici, sperimentali e di intervento emergono dati contrastanti riguardo il beneficio dei PUFA n-3 sulla prevenzione dell'infarto miocardico.

- Le ipotesi sono molte e sono necessari ulteriori ampi trials clinici randomizzati per chiarirle.

- Attualmente possiamo solo consigliare una dieta ricca in PUFA n-3 e riservare l'integrazione di 
PUFA n-3 ai soggetti che non gradiscono e/o tollerano tale dieta.

\section{Bibliografia}

1. Bang HO, Dyerberg J. Lipid metabolism and ischemic heart disease in Greenland Eskimos. In: Draper H, ed. Advances in nutrition research. New York: Plenum Press; 1980. pp 1-22.

2. Albert CM, Hennekens CH, O'Donnell CJ, et al. Fish consumption and risk of sudden cardiac death. JAMA 1998;279:23-8.

3. Mozaffarian D, Lemaitre RN, Kuller LH, et al. Cardiac benefits of fish consumption may depend on the type of fish meal consumed: the Cardiovascular Health Study. Circulation 2003;107:1372-7.

4. Mozaffarian D, Ascherio A, Hu FB, et al. Interplay between different polyunsaturated fatty acids and risk of coronary heart disease in men. Circulation 2005;111: 157-64.

5. Iso $\mathrm{H}$, Kobayashi $\mathrm{M}$, Ishihara J, et al. Intake of fish and n3 fatty acids and risk of coronary heart disease among Japanese: the Japan Public Health Center-Based (JPHC) Study Cohort I. Circulation 2006;113:195-202.

6. Strand E, Pedersen ER, Svingen GFT, et al. Dietary intake of n-3 long-chain polyunsaturated fatty acids and risk of myocardial infarction in coronary artery disease patients with or without diabetes mellitus: a prospective cohort study. BMC Med 2013;11:216.

7. Burr ML, Fehily AM, Gilbert JF, et al. Effects of changes in fat, fish, and fibre intakes on death and myocardial reinfarction: diet and reinfarction trial (DART). Lancet 1989;2:757-61.

8. [No authors listed]. Dietary supplementation with n-3 polyunsaturated fatty acids and vitamin $\mathrm{E}$ after myocardial infarction: results of the GISSI-prevenzione trial. Gruppo Italiano per lo Studio della Sopravvivenza nell'Infarto Miocardico. Lancet 1999;354:447-55.

9. Yokoyama M, Origasa H, Matsuzaki M, et al. Effects of eicosapentaenoic acid on major coronary events in hypercholesterolemic patients (JELIS): a randomized open-label, blinded, end-point analysis. Lancet 2007; 369:1090-8.

10. Gissi-HF Investigators, Tavazzi L, Maggioni AP, et al. Effect of $n-3$ polyunsaturated fatty acids in patients with chronic heart failure (the GISSI-HF trial): a randomised, double-blind, placebo-controlled trial. Lancet 2008; 372:1223-30.

11. Burr ML, Ashfield-Watt PA, Dunstan FD, et al. Lack of benefit of dietary advice to men with angina: results of a controlled trial. Eur J Clin Nutr 2003;57:193-200.

12. Kromhout D, Giltay EJ, Geleijnse JM; Alpha Omega Trial Group. n-3 fatty acids and cardiovascular events after myocardial infarction. N Engl J Med 2010;363: 2015-26

13. Rauch B, Schiele R, Schneider S, et al. OMEGA, a randomized, placebo-controlled trial to test the effect of highly purified omega-3 fatty acids on top of modern guideline-adjusted therapy after myocardial infarction. Circulation 2010;122:2152-9.

14. Kromhout D, Geleijnse JM, de Goede J, et al. n-3 fatty acids, ventricular arrhythmia-related events, and fatal myocardial infarction in postmyocardial infarction patients with diabetes. Diabetes Care 2011;34:2515-20.

15. ORIGIN Trial Investigators; Bosch J, Gerstein HC, et al. n-3 fatty acids and cardiovascular outcomes in patients with dysglycemia. N Engl J Med 2012;367:309-18.

16. Writing Group for the AREDS2 Research Group, Bonds DE, Harrington M, et al. Effect of long-chain w-3 fatty acids and lutein + zeaxanthin supplements on cardiovascular outcomes: results of the age-related eye disease study 2 (AREDS2) randomized clinical trial. JAMA Intern Med 2014;174:763-71.

17. Rizos EC, Ntzani EE, Bika E, et al. Association between omega-3 fatty acid supplementation and risk of major cardiovascular disease events: a systematic review and meta-analysis. JAMA 2012;308:1024-33.

18. Wen YT, Daj JH, Gao Q. Effects of omega-3 fatty acid on major cardiovascular events and mortality in patients with coronary heart disease: a meta-analysis of randomized controlled trials. Nutr Metabolic Cardiovasc Dis 2014;24:470-5.

19. Enns JE, Yaganeh A, Zarychanski R, et al. The impact of omega-3 polyunsaturated fatty acid supplementation on the incidence of cardiovascular events and complications in peripheral arterial disease: a systemic review and meta-analysis. BMC Cardiovasc Disord 2014;14:70.

20. Geleijnse JM, Giltay EJ, Grobbee DE, et al. Blood pressure response to fish oil supplementation: metaregression analysis of randomized trials. J Hypertens 2002; 20:1493-9

21. Mozaffarian D, Gottdiener JS, Siscovick DS. Intake of tuna or other broiled or baked fish versus fried fish and cardiac structure, function, and hemodynamics. Am J Cardiol 2006;97:216-22.

22. Demaison L, Blet J, Sergiel JP, et al. Effect of dietary polyunsaturated fatty acids on contractile function of hearts isolated from sedentary and trained rats. Reprod Nutr Dev 2000;40:113-25.

23. Omura M, Kobayashi S, Mizukami Y, et al. Eicosapentaenoic acid (EPA) induces $\mathrm{Ca}(2+)$-independent activation and translocation of endothelial nitric oxide synthase and endothelium-dependent vasorelaxation. FEBS Lett 2001;487:361-6.

24. De Caterina R, Liao JK, Libby P. Fatty acid modulation of endothelial activation. Am J Clin Nutr 2000;71:213S$23 \mathrm{~S}$

25. Li Q, Zhang Q, Wang M, et al. Eicosapentaenoic acid modifies lipid composition in caveolae and induces translocation of endothelial nitric oxide synthase. Biochimie 2007;89:169-77

26. Li Q, Zhang Q, Wang M, et al. Docosahexaenoic acid affects endothelial nitric oxide synthase in caveolae. Arch Biochem Biophys 2007;466:250-9.

27. Harris WS. n-3 fatty acids and serum lipoproteins: human studies. Am J Clin Nutr 1997;65:1645S-54S.

28. Balk EM, Lichtenstein AH, Chung M, et al. Effects of omega-3 fatty acids on serum markers of cardiovascular disease risk: a systematic review. Atherosclerosis 2006; 189:19-30.

29. Wang C, Chung M, Lichtenstein A, et al. Effects of omega-3 fatty acids on cardiovascular disease. Evid Rep Technol Assess (Summ) 2004;94:1-8.

30. Friedberg CE, Janssen MJ, Heine RJ, Grobbee DE. Fish 
oil and glycemic control in diabetes. A meta-analysis. Diabetes Care 1998;21:494-500.

31. Minihane AM, Khan S, Leigh-Firbank EC, et al. ApoE polymorphism and fish oil supplementation in subjects with an atherogenic lipoprotein phenotype. Arterioscler Thromb Vasc Biol 2000;20:1990-7.

32. Griffin MD, Sanders TA, Davies IG, et al. Effects of altering the ratio of dietary n- 6 to n-3 fatty acids on insulin sensitivity, lipoprotein size, and postprandial lipemia in men and postmenopausal women aged $45-70$ y: the OPTILIP study. Am J Clin Nutr 2006;84:1290-8.

33. Mozaffarian D, Geelen A, Brouwer IA, et al. Effect of fish oil on heart rate in humans: a meta-analysis of randomized controlled trials. Circulation 2005;112:1945-52.

34. McLennan PL. Myocardial membrane fatty acids and the antiarrhythmic actions of dietary fish oil in animal models. Lipids 2001;36:S111-4.

35. Leaf A, Kang JX, Xiao YF, et al. Clinical prevention of sudden cardiac death by n-3 polyunsaturated fatty acids and mechanism of prevention of arrhythmias by n-3 fish oils. Circulation 2003;107:2646-52.

36. O'Keefe JH Jr, Abuissa H, Sastre A, et al. Effects of omega-3 fatty acids on resting heart rate, heart rate recovery after exercise, and heart rate variability in men with healed myocardial infarctions and depressed ejection fractions. Am J Cardiol 2006;97:1127-30.

37. James MJ, Gibson RA, Cleland LG. Dietary polyunsaturated fatty acids and inflammatory mediator production. Am J Clin Nutr 2000;71:343S-8S.
38. Lucas M, Kimmig MD, Dean G, et al. Do omega-3 polyunsaturated fatty acids prevent cardiovascular disease? A review of the randomized clinical trials. Lipid Insights 2013;6:13-20.

39. Kromhout D, de Goede J. Update on cardiometabolic health effects of omega-3 fatty acids. Curr Opin Lipidol 2014;25:85-90.

40. De Caterina R. n-3 fatty acids in cardiovascular disease. N Engl J Med 2011;364:2439-50.

41. McLennan PL. Cardiac physiology and clinical efficacy of dietary fish oil carified through cellular mechnisms of omega-3 polyunsaturated fatty acids. Eur J Appl Physiol 2014;114:1333-56.

42. Superko HR, Superko AR, Lundberg GP, et al. Omega3 fatty acid blood levels clinical significance update. Curr Cardiovasc Risk Rep 2014;8:407.

43. Moltó-Puigmartí FC, Plat J, Mensink RP, et al. FADS1 FADS2 gene variants modify the association between fish intake and the docosahexaenoic acid proportions in human milk. Am J Clin Nutr 2010;91:1368-76.

44. Poole CD, Halcox JP, Jenkins-Jones S, et al. Omega-3 fatty acids and mortality outcome in patients with and without type 2 diabetes after myocardial infarction: a retrospective, matched-cohort study. Clin Ther 2013;35: 40-51.

45. Nestel P, Clifton P, Colquhoun D, et al. Indications for omega-3 long chain polyunsaturated fatty acid in the prevention and treatment of cardiovascular disease. Heart Lung Circulation 2015 [in press]. 


\title{
Omega-3 e scompenso cardiaco
}

\author{
Riccardo Gerloni \\ Medicina Interna, Ospedale Cattinara, Trieste, Italia
}

Nonostante i progressi nella diagnosi e nel trattamento dello scompenso cardiaco, la morbilità e la mortalità di questa sindrome sono in continua crescita. ${ }^{1,2}$ L'individuazione di molecole nuove, che siano in grado di ridurre la mortalità dello scompenso cardiaco, resta un obiettivo molto ambito ed è per questo che gli acidi grassi omega-3 (PUFA n-3) destano oggi grande attenzione. In effetti, in letteratura vi sono alcune pubblicazioni che dimostrano come il consumo di PUFA n-3 riduca l'incidenza di scompenso cardiaco e pochi trials randomizzati che evidenziano come la supplementazione con PUFA n-3 possa migliorare la prognosi di pazienti affetti da scompenso cardiaco.

L'associazione tra consumo di pesce, l'alimento con maggior contenuto di PUFA n-3, e il rischio di scompenso cardiaco è stato per la prima volta descritto da Mozzaffarian e coll. nel 2005. ${ }^{3}$ Attraverso 12 anni di follow-up è emerso che il consumo di pesce era inversamente correlato all'incidenza di scompenso cardiaco. Una riduzione del rischio pari al 32\% emergeva confrontando gli individui che mangiavano pesce 5 volte settimana, rispetto a quelli che lo mangiavano meno di una volta al mese. Tale studio quantificava l'introito di pesce attraverso un questionario e, quindi, è stato soggetto a critiche. Infatti l'assorbimento e il metabolismo dei PUFA n-3 varia tra gli individui ${ }^{4}$ ed è forse questo uno dei motivi che può spiegare la difformità di risultati tra gli altri studi prospettici osservazionali. ${ }^{5-7}$ Mozzafarian ${ }^{8}$ ha pubblicato allora più recentemente un lavoro che dimostrava come il livello circolante di PUFA n-3, e non lo stile di alimentazione, correlava inversamente con l'incidenza di scompenso cardiaco. Anche lo studio ARIC (atherosclerosis risk in communities $)^{9}$ con un follow-up lungo oltre 14 anni

Corrispondente: Riccardo Gerloni, Medicina Interna, Ospedale Cattinara, strada di Fiume 447, 34149 Trieste, Italia. E-mail: riccardogerloni@libero.it

Articolo pubblicato secondo la Creative Commons Attribution NonCommercial 3.0 License (CC BY-NC 3.0).

(C) Copyright R. Gerloni, 2015

Licensee PAGEPress, Italy

QUADERNI - Italian Journal of Medicine 2015; 3:274-278 ha analizzato i livelli circolanti di PUFA n-3 ed è arrivato a conclusioni analoghe relativamente all'incidenza di scompenso nelle donne, non negli uomini. Questa incertezza di risultati ha stimolato Djousse e coll. ${ }^{10}$ a produrre una metanalisi, dimostrando come i PUFA n-3 siano efficaci nel ridurre l'incidenza di scompenso cardiaco. Studi clinici randomizzati volti a verificare se la supplementazione con PUFA n-3 possa ridurre l'incidenza di scompenso cardiaco sono scarsi e la poca evidenza di un effetto favorevole deriva dal lavoro di Hooper e coll. ${ }^{11}$ Sono necessari quindi ulteriori studi per chiarire l'argomento.

Evidenze più solide derivano invece dai trials condotti sui pazienti con storia di scompenso cardiaco tanto che le ultime linee guida della Società Europea di Cardiologia suggeriscono di considerare i PUFA n-3 tra i farmaci della terapia dello scompenso cardiaco. ${ }^{12}$ Lo studio del Gruppo Italiano per lo Studio della Sopravvivenza nell'Infarto Miocardico (GISSI-HF), ${ }^{13}$ importante trial clinico randomizzato su oltre 7000 pazienti con scompenso cardiaco affetti da cardiopatie diverse, ha dimostrato che i soggetti in terapia con un grammo/die di PUFA n-3 dopo 3,9 anni di follow-up presentavano un rischio assoluto di riduzione della mortalità totale o ammissione in ospedale per motivi cardiovascolari pari a $9 \%$ con maggior beneficio nei diabetici e nelle persone anziane. Le curve di Kaplan-Meier tra trattati e non cominciavano a divergere a 30 mesi per la mortalità totale e a 24 mesi se si considerava anche le amissioni in ospedale per motivi cardiovascolari. In pratica questo significa che trattare 1000 pazienti per 3,9 anni evita 18 decessi e 17 ricoveri per instabilizzazione di patologia cardiovascolare. Dalle analisi degli outcomes secondari, inoltre, non emergeva nei trattati un beneficio significativo sull'incidenza di ictus, infarto miocardico, morte improvvisa o ricovero per scompenso cardiaco. Si assisteva invece ad una riduzione statisticamente significativa sulla morte cardiovascolare e l'ospedalizzazione per motivi cardiovascolari, come illustrato in Tabella 1. I risultati dello studio GISSI-HF, quindi, corroborano l'ipotesi, già apparsa in letteratura, secondo la quale il beneficio dei PUFA n-3 si esplicherebbe in gran parte sulla riduzione degli eventi aritmici. Infatti la gran parte della riduzione del rischio assoluto sulla 
mortalità totale veniva attribuito alla morte aritmica e circa la metà della riduzione di rischio assoluto sul ricovero per cause cardiovascolari veniva ascritto ad aritmie ventricolari. In pratica su 1000 pazienti trattati con PUFA n-3 venivano evitate 9 morti aritmiche e 8 ammissioni in ospedale per aritmie. Va ricordato che tali risultati potrebbero rappresentare una sottostima, poiché la compliance dimostrata nello studio è stata bassa (28,7\% nei trattati e $29,6 \%$ nel placebo), aspetto tuttavia da valorizzare per l'affinità al mondo reale. Merita infine sottolineare che l'effetto protettivo dei PUFA n-3 nello studio GISSI-HF si manifestava più lentamente rispetto ai trial che includevano pazienti reduci da infarto miocardico. Tale aspetto può essere ascritto alla minore propensione alle aritmie di un paziente con scompenso cardiaco stabilizzato piuttosto che uno reduce da recente evento coronarico. $^{14}$

Oltre alla ragionevole ipotesi che i PUFA n-3 siano efficaci nel paziente con scompenso per la loro azione antiaritmica, esistono tutta una serie di lavori che ascrivono il beneficio di questi farmaci a meccanismi diversi. Mozzafarian e coll. ${ }^{15}$ ha ipotizzato un effetto sul riempimento diastolico con conseguente incremento della portata cardiaca, Nodari e coll. ${ }^{16}$ in pazienti con cardiopatia dilatativa non ischemica ha trovato un aumento della funzionalità ventricolare sistolica e della capacità funzionali dei pazienti. Anche Moerti e coll., ${ }^{17}$ che hanno analizzato pazienti con scompenso cardiaco senza evidenza di cardiopatia ischemica, randomizzandoli a trattamento con 1 grammo/die o $4 \mathrm{~g} / \mathrm{die}$ di PUFA n3 o placebo, hanno dimostrato in soli 3 mesi di follow-up un aumento della frazione d'eiezione dose-dipendente. Anche una sottoanalisi dello studio GISSI-HF ${ }^{18}$ apparso nel 2010 ha evidenziato che $i$ soggetti in terapia con PUFA n-3 presentavano un miglioramento della funzionalità ventricolare sinistra. Tali effetti sulla performance ventricolare sinistra trovano spiegazioni in vari studi sperimentali che dimostrano come i PUFA n-3 siano efficaci sulle proteine contrattili e sull'accoppiamento eccitazione-contrazione con conseguente miglioramento della forza di contrazione ventricolare. ${ }^{19-22}$ Altri studi hanno dimostrato come i PUFA n-3 possano migliorare il metabolismo energetico cardiaco, ${ }^{22,23}$ ridurre o prevenire l'ipertrofia cardiaca, ${ }^{24,25}$ modulare il sistema $\beta$ adrenergico. ${ }^{26-30}$

Vi sono infine numerose segnalazioni riguardo al possibile effetto dei PUFA n-3 sul letto vascolare arterioso, tra cui miglioramento della disfunzione endoteliale, ${ }^{31-35}$ riduzione delle resistenze sistemiche $^{15}$ per aumento della produzione di ossido nitrico, ${ }^{36-39}$ riduzione della risposta vasocostrittrice alla noradrenalina e angiotensina II, ${ }^{40}$ incremento della compliance arteriolare, ${ }^{41}$ della risposta vasodilatatoria ${ }^{40} \mathrm{e}$, infine, riduzione della produzione di endotelina $1,{ }^{42}$ un potente noto vasocostrittore, associato ad una prognosi scarsa nei pazienti con scompenso cardiaco.

Infine l'effetto benefico dei PUFA n-3 sui pazienti con scompenso cardiaco potrebbe essere ottenuto grazie all'azione modulatrice sul sistema neuro-ormonale ${ }^{43-47}$ e a quella antiinfiammatoria. ${ }^{48-60}$ È noto infatti come l'infiammazione e l'attivazione del sistema neuroormonale contribuiscano alla progressione dello scompenso cardiaco.

In conclusione si può affermare che la supplementazione con PUFA n-3 ha fornito alcuni risultati positivi nei pazienti con scompenso cardiaco con un meccanismo verosimilmente antiaritmico. Numerosi lavori indicano benefici dei PUFA n-3 anche su meccanismi fisiopatologici diversi. Essendo lo scompenso cardiaco un crocevia che origina da cardiopatie diverse, analizzare con ampi trial randomizzati pazienti con genesi identiche sarebbe metodologicamente corretto e potrebbe portare a risultati diversi e inattesi. Se consideriamo l'effetto antiaritmico come preponderante, è probabile che nell'ambito dei pazienti con scompenso cardiaco quelli con disfunzione ventricolare sinistra ne beneficerebbero di più.

Tabella 1. Esiti degli outcomes nello studio GISSI-HF.

\begin{tabular}{lcc}
\hline Outcome & Rischio relativo & Significatività $(\mathbf{P}<)$ \\
\hline Morte per tutte le cause & $0,91(0,883-0,998)$ & 0,041 \\
\hline Morte per tutte le cause o ricovero per cause cardiovascolari & $0,92(0,849-0,999)$ & 0,009 \\
\hline Morte cardiovascolare & $0,9(0,81-0,99)$ & 0,045 \\
\hline Ricovero per cause cardiovascolari & $0,93(0,87-0,99)$ & 0,026 \\
\hline Ricovero per scompenso cardiaco & $0,94(0,86-1,02)$ & 0,147 \\
\hline Morte aritmica & $0,93(0,79-1,08)$ & 0,333 \\
\hline Infarto miocardico & $0,82(0,63-1,06)$ & 0,121 \\
\hline Stroke & $1,16(0,89-1,51)$ & 0,271 \\
\hline
\end{tabular}




\section{Messaggi chiave}

- Non vi è evidenza certa di efficacia dei PUFA n3 sulla riduzione dell'incidenza di scompenso cardiaco.

- Nei pazienti con scompenso cardiaco l'effetto favorevole dei PUFA n-3 potrebbe esplicarsi principalmente sul versante antiaritmico.

- Necessari studi ad hoc per verificare l'effetto di questi farmaci su modelli fisiopatologici omogenei nei pazienti con scompenso cardiaco.

\section{Bibliografia}

1. Braunwald E, Bristow MR. Congestive heart failure: fifty years of progress. Circulation 2000;102:IV14-23.

2. Roger VL, Go AS, Lloyd-Jones DM, et al. Heart disease and stroke statistics-2011 update: a report from the American Heart Association. Circulation 2011;123:e18-e209.

3. Mozaffarian D, Bryson CL, Lemaitre RN, et al. Fish intake and risk of incident heart failure. J Am Coll Cardiol 2005;45:2015-21.

4. Superko HR, Superko AR, Lundberg GP, et al. Omega3 fatty acid blood levels clinical significance update. Curr Cardiovasc Risk Rep 2014;8:407.

5. Levitan EB, Wolk A, Mittleman MA. Fish consumption, marine omega-3 fatty acids, and incidence of heart failure: a population-based prospective study of middleaged and elderly men. Eur Heart J 2009;30:1495-500.

6. Dijkstra SC, Brouwer IA, van Rooij FJ, et al. Intake of very long chain $n-3$ fatty acids from fish and the incidence of heart failure: the Rotterdam Study. Eur J Heart Fail 2009;11:922-8.

7. Yamagishi K, Iso H, Date C, et al. Fish, omega-3 polyunsaturated fatty acids, and mortality from cardiovascular diseases in a nationwide community-based cohort of Japanese men and women the JACC (Japan Collaborative Cohort Study for Evaluation of Cancer Risk) Study. J Am Coll Cardiol 2008;52:988-96.

8. Mozaffarian D, Lemaitre R, King I, et al. Circulating long-chain omega-3 fatty acids and incidence of congestive heart failure in older adults: the cardiovascular health study. Ann Intern Med 2011;155:160-70.

9. Yamagishi K, Nettleton JA, Folsom AR. Plasma fatty acid composition and incident heart failure in middleaged adults: the atherosclerosis risk in communities (ARIC) Study. Am Heart J 2008;156:965-74.

10. Djousse L, Akinkuolie AO, Wu JH, et al. Fish consumption, omega-3 fatty acids and risk of heart failure: a meta-analysis. Clin Nutr 2012;31:846-53.

11. Hooper L, Thompson RL, Harrison RA, et al. Omega-3 fatty acids for prevention and treatment of cardiovascular disease. Cochrane Database Syst Rev 2004;(4): CD003177.

12. McMurray JJ, Adamopoulos S, Anker SD, et al. ESC guidelines for the diagnosis and treatment of acute and chronic heart failure 2012: The Task Force for the Diagnosis and Treatment of Acute and Chronic Heart Failure 2012 of the European Society of Cardiology. Developed in collaboration with the Heart Failure Association (HFA) of the ESC. Eur Heart J 2012;33:1787-847.
13. Gissi-HF Investigators, Tavazzi L, Maggioni AP, et al. Effect of $n-3$ polyunsaturated fatty acids in patients with chronic heart failure (the GISSI-HF trial): a randomised, double-blind, placebo-controlled trial. Lancet 2008;372: 1223-30.

14. Marchioli R, Levantesi G. n-3 PUFAs and heart failure. Int J Cardiol 2013;170:S28-S32.

15. Mozaffarian D, Gottdiener JS, Siscovick DS. Intake of tuna or other broiled or baked fish versus fried fish and cardiac structure, function, and hemodynamics. Am J Cardiol 2006;97:216-22.

16. Nodari S, Triggiani M, Campia U, et al. Effects of n-3 polyunsaturated fatty acids on left ventricular function and functional capacity in patients with dilated cardiomyopathy. J Am Coll Cardiol 2011;57:870-9.

17. Moertl D, Hammer A, Steiner S, et al. Dose-dependent effects of omega-3-polyunsaturated fatty acids on systolic left ventricular function, endothelial function, and markers of inflammation in chronic heart failure of nonischemic origin: a double-blind, placebo-controlled, 3arm study. Am Heart J 2011;161:915.e1-9.

18. Ghio S, Scelsi L, Latini R et al. Effects of n-3 polyunsaturated fatty acids and of rosuvastatin on left ventricular function in chronic heart failure: a substudy of GISSI-HF trial. Eur J Heart Fail 2010;12:1345-53.

19. Paige JA, Liao R, Hajjar RJ, et al. Effect of a high omega-3 fatty acid diet on cardiac contractile performance in Oncorhynchus mykiss. Cardiovasc Res 1996;31: 249-62.

20. Rodrigo GC, Dhanapala S, Macknight AD. Effects of eicosapentaenoic acid on the contraction of intact, and spontaneous contraction of chemically permeabilized mammalian ventricular myocytes. J Mol Cell Cardiol 1999;31:733-43.

21. Oddis $\mathrm{CV}$, Mayer $\mathrm{OH}$, Finkel MS. Inotropic, chronotropic, and radioligand binding characteristics of leukotriene B4 in cardiac myocyte, papillary muscle, and membrane preparations. Prostaglandins Leukot Essent Fatty Acids 1996;54:223-8.

22. Duda MK, O'Shea KM, Tintinu A, et al. Fish oil, but not flaxseed oil, decreases inflammation and prevents pressure overload-induced cardiac dysfunction. Cardiovasc Res 2009;81:319-27.

23. McLennan PL, Barnden LR, Bridle TM et al. Dietary fat modulation of left ventricular ejection fraction in the marmoset due to enhanced filling. Cardiovasc Res 1992; 26:871-7.

24. Denys A, Hichami A, Maume B, et al. Docosahexaenoic acid modulates phorbol ester-induced activation of extracellular signal-regulated kinases 1 and 2 in NIH/3T3 cells. Lipids 2001;36:813-8.

25. Denys A, Hichami A, Khan NA. Eicosapentaenoic acid and docosahexaenoic acid modulate MAP kinase enzyme activity in human T-cells. Mol Cell Biochem 2002;232:143-8.

26. Alam SQ, Ren YF, Alam BS. [3H]forskolin- and [3H]dihydroalprenolol-binding sites and adenylate cyclase activity in heart of rats fed diets containing different oils. Lipids 1988;23:207-13.

27. Patten GS, Rinaldi JA, McMurchie EJ. Effects of dietary eicosapentaenoate (20:5 n-3) on cardiac $\beta$-adrenergic receptor activity in the marmoset monkey. Biochem Biophys Res Commun 1989;162:686-93. 
28. Grynberg A, Fournier A, Sergiel JP, et al. Effect of docosahexaenoic acid and eicosapentaenoic acid in the phospholipids of rat heart muscle cells on adrenoceptor responsiveness and mechanism. J Mol Cell Cardiol 1995;27:2507-20.

29. Grynberg A, Fournier A, Sergiel JP, et al. Membrane docosahexaenoic acid vs. eicosapentaenoic acid and the beating function of the cardiomyocyte and its regulation through the adrenergic receptors. Lipids 1996;31:S205-10

30. Delerive P, Oudot F, Ponsard B, et al. Hypoxia-reoxygenation and polyunsaturated fatty acids modulate adrenergic functions in cultured cardiomyocytes. J Mol Cell Cardiol 1999;31:377-86.

31. Goodfellow J, Bellamy MF, Ramsey MW, et al. Dietary supplementation with marine omega-3 fatty acids improve systemic large artery endothelial function in subjects with hypercholesterolemia. J Am Coll Cardiol 2000;35:265-70

32. von Schacky C. The role of omega-3 fatty acids in cardiovascular disease. Curr Atheroscler Rep 2003;5: 139-45.

33. Engler MM, Engler MB, Malloy M, et al. Docosahexaenoic acid restores endothelial function in children with hyperlipidemia: results from the EARLY study. Int J Clin Pharmacol Ther 2004;42:672-9.

34. Robinson JG, Stone NJ. Antiatherosclerotic and antithrombotic effects of omega-3 fatty acids. Am J Cardiol 2006;98:39i-49i.

35. Matsumoto T, Nakayama N, Ishida K, et al. Eicosapentaenoic acid improves imbalance between vasodilator and vasoconstrictor actions of endothelium-derived factors in mesenteric arteries from rats at chronic stage of type 2 diabetes. J Pharmacol Exp Ther 2009;329:324-34.

36. Shimokawa H, Lam JY, Chesebro JH, et al. Effects of dietary supplementation with cod-liver oil on endothelium-dependent responses in porcine coronary arteries. Circulation 1987;76:898-905.

37. Harris WS, Rambjor GS, Windsor SL, et al. n-3 fatty acids and urinary excretion of nitric oxide metabolites in humans. Am J Clin Nutr 1997;65:459-64.

38. Omura M, Kobayashi S, Mizukami Y, et al. Eicosapentaenoic acid (EPA) induces $\mathrm{Ca} 2+$-independent activation and translocation of endothelial nitric oxide synthase and endothelium-dependent vasorelaxation. FEBS Lett 2001;487:361-6

39. Hirafuji M, Machida T, Tsunoda M, et al. Docosahexaenoic acid potentiates interleukin- $1 \beta$ induction of nitric oxide synthase through mechanism involving p44/42 MAPK activation in rat vascular smooth muscle cells. Br J Pharmacol 2002;136:613-9.

40. Mori TA, Watts GF, Burke V, et al. Differential effects of eicosapentaenoic acid and docosahexaenoic acid on vascular reactivity of the forearm microcirculation in hyperlipidemic, overweight men. Circulation 2000;102: 1264-9.

41. McVeigh GE, Brennan GM, Cohn JN, et al. Fish oil improves arterial compliance in non-insulin-dependent diabetes mellitus. Arterioscler Thromb 1994;14: 1425-9.

42. Kohno M, Ohmori K, Wada Y, et al. Inhibition by eicosapentaenoic acid of oxidized-LDL- and lysophosphatidylcholine-induced human coronary artery smooth muscle cell production of endothelin. J Vasc Res 2001; 38:379-88

43. Christensen JH, Gustenhoff P, Korup E, et al. Effect of fish oil on heart rate variability in survivors of myocardial infarction: a double blind randomized controlled trial. BMJ 1996;312:677-8.

44. Christensen JH, Christensen MS, Dyerberg J, et al. Heart rate variability and fatty acid content of blood cell membranes: a dose-response study with n-3 fatty acids. Am J Clin Nutr 1999;70:331-7.

45. Engler MM, Engler MB, Goodfriend TL, et al. Docosahexaenoic acid is an antihypertensive nutrient that affects aldosterone production in SHR. Proc Soc Exp Biol Med 1999;221:32-8.

46. Miyazaki M, Takemura N, Watanabe S, et al. Dietary docosahexaenoic acid ameliorates, but rapeseed oil and safflower oil accelerate renal injury in stroke-prone spontaneously hypertensive rats as compared with soybean oil, which is associated with expression for renal transforming growth factor- $\beta$, fibronectin and renin. Biochim Biophys Acta 2000;1483:101-10.

47. Jin Y, Wu JX, Hong M, et al. The effects of streptozotocin induced diabetes mellitus and fish oil compound on gene expression of atrial natriuretic peptide in rat. Comp Biochem Physiol A Comp Physiol 1991;100:221-5.

48. Goldman DW, Pickett WC, Goetzl EJ. Human neutrophil chemotactic and degranulating activities of leukotriene B5 (LTB5) derived from eicosapentaenoic acid. Biochem Biophys Res Commun 1983;117:282-8.

49. James MJ, Cleland LG, Gibson RA, et al. Interaction between fish and vegetable oils in relation to rat leucocyte leukotriene production. J Nutr 1991;121:631-7.

50. Hawkes JS, James MJ, Cleland LG. Separation and quantification of PGE3 following derivatization with panacyl bromide by high pressure liquid chromatography with fluorometric detection. Prostaglandins 1991; 42:355-68.

51. Hawkes JS, James MJ, Cleland LG. Biological activity of prostaglandin E3 with regard to oedema formation in mice. Agents Actions 1992;35:85-7.

52. Calder PC. Omega-3 polyunsaturated fatty acids, inflammation and immunity. World Rev Nutr Diet 2001;88:109-16

53. Serhan CN, Clish CB, Brannon J, et al. Novel functional sets of lipid-derived mediators with antiinflammatory actions generated from omega-3 fatty acids via cyclooxygenase 2-nonsteroidal antiinflammatory drugs and transcellular processing. J Exp Med 2000;192:1197-204.

54. Serhan CN, Clish CB, Brannon J, et al. Antimicroinflammatory lipid signals generated from dietary N-3 fatty acids via cyclooxygenase- 2 and transcellular processing: a novel mechanism for NSAID and N-3 PUFA therapeutic actions. J Physiol Pharmacol 2000;51:643-54.

55. Mukherjee PK, Marcheselli VL, Serhan CN, et al. Neuroprotectin D1: a docosahexaenoic acid-derived docosatriene protects human retinal pigment epithelial cells from oxidative stress. Proc Natl Acad Sci U S A 2004;101:8491-6.

56. Serhan CN, ChiangN, Van Dyke TE. Resolving inflammation: dual anti-inflammatory and pro-resolution lipid mediators. Nat Rev Immunol 2008;8:349-61.

57. Mayer K, Merfels M, Muhly-Reinholz M, et al. Omega3 fatty acids suppress monocyte adhesion to human en- 
dothelial cells: role of endothelial PAF generation. Am J Physiol Heart Circ Physiol 2002;283:H811-8.

58. Kaminski WE, Jendraschak E, Kiefl R, et al. Dietary omega-3 fatty acids lower levels of platelet-derived growth factor mRNA in human mononuclear cells. Blood 1993;81:1871-9.

59. Mehra MR, Lavie CJ, Ventura HO, et al. Fish oils pro- duce anti-inflammatory effects and improve body weight in severe heart failure. J Heart Lung Transplant 2006;25:834-8.

60. Endres S, Ghorbani R, Kelley VE, et al. The effect of dietary supplementation with n-3 polyunsaturated fatty acids on the synthesis of interleukin-1 and tumor necrosis factor by mononuclear cells. N Engl J Med 1989;320:265-71. 
Farmaci nella forma migliore

\title{
I prodotti naturali: una possibile alternativa alle statine per la riduzione del colesterolo
}

\author{
Maria Cristina Pasquini \\ UO Oncologia Medica, Ospedale Maggiore, Crema (CR), Italia
}

\section{Introduzione}

La dislipidemia accelera i processi di aterosclerosi e risulta essere il principale fattore di rischio per incidenza e mortalità di eventi coronarici. L'adeguato controllo dei livelli di lipidi sierici permette di ottenere la riduzione del profilo di rischio cardiovascolare individuale. $^{1}$

Le statine rappresentano il trattamento di scelta evidence-based per ridurre i livelli di colesterolo con lipoproteine a bassa densità (LDL). Tuttavia, nella pratica comune spesso si osserva un mancato raggiungimento del target ottimale dei livelli di colesterolo. ${ }^{2} \mathrm{Il}$ National Health and Nutrition Survey (NHANES) ha evidenziato come tra gli adulti trattati in prevenzione cardiovascolare, l'ipercolesterolemia sia il fattore di rischio gestito più frequentemente in modo sub ottimale. ${ }^{3}$ Nei pazienti in terapia, inoltre, non sono infrequenti interruzioni del trattamento a causa di eventi avversi e intolleranza, quali mialgie e tossicità epatica. ${ }^{4,5}$ Particolare attenzione deve inoltre essere rivolta alla categoria di individui a basso rischio cardiovascolare con livelli subottimali di colesterolo, dove il trattamento con statine costituisce ad oggi materia di intenso dibattito. ${ }^{6,7}$

Le linee guida attribuiscono all'intervento sugli stili di vita un ruolo chiave nel trattamento dei pazienti con ipercolesterolemia. ${ }^{8}$ Dal punto di vista dietetico l'obiettivo è rivolto alla riduzione dell'introito di cibi con effetti negativi sul profilo lipidico; i grassi non dovrebbero superare il $35 \%$ del totale delle calorie introdotte, riducendo i grassi saturi a meno del $6 \%$ e non

Corrispondente: Maria Cristina Pasquini, UO Oncologia Medica, Ospedale Maggiore di Crema, l.go U. Dossena 2, 26013 Crema (CR), Italia.

E-mail: mcristinapasquini@libero.it

Articolo pubblicato secondo la Creative Commons Attribution NonCommercial 3.0 License (CC BY-NC 3.0).

CCopyright M.C. Pasquini, 2015

Licensee PAGEPress, Italy

QUADERNI - Italian Journal of Medicine 2015; 3:279-287 superando l'introito giornaliero di $200 \mathrm{mg}$ di colesterolo. È noto che molti nutrienti, contenuti nel pesce, frutta, verdura e nocciole, hanno un effetto positivo sul metabolismo del colesterolo; l'assunzione di questi nutrienti può essere incrementata, ma per raggiungere un effetto terapeutico può essere utile supplementare la dieta con cibi arricchiti o con nutraceutici. ${ }^{9}$

I nutraceutici e i cibi funzionali possono dunque rappresentare un'utile opportunità nel trattamento della dislipidemia, sebbene non completamente chiarito, il loro meccansimo d'azione sarebbe riconducibile alla riduzione della $7-\alpha$ idrossilasi, all'aumento dell'escrezione fecale di colesterolo, alla riduzione dei livelli del m-RNA della 3-idrossi-3 metilglutaril-CoA reduttasi e alla riduzione della secrezione di colesterolo LDL. ${ }^{10-12}$

\section{Nutraceutici}

Il termine nutraceutico è un neologismo chimerico risultante dalla fusione di nutrizione e farmaceutica, venne introdotto per la prima volta nel 1989 dal dott. De Felice in riferimento ad alimenti dotati di un presumibile beneficio sulla salute dell'uomo, inclusa la prevenzione e/o il trattamento di una malattia. ${ }^{13}$

La storia delle civiltà ci insegna come Indiani, Egizi, Cinesi, e Sumeri utilizzassero gli alimenti per curare e prevenire le malattie, lo stesso Ippocrate, considerato il padre della medicina occidentale, sentenziava: Lasciate che il cibo sia la vostra medicina e la vostra medicina sia il cibo.

Le sostanze nutraceutiche sono normalmente derivate dalle piante, dagli alimenti e da fonti microbiche. Esempi di nutraceutici sono i probiotici, gli antiossidanti, gli acidi grassi polinsaturi (omega-3, omega-6), le vitamine e i complessi enzimatici.

Il termine nutraceutico è strettamente correlato a quello di alimento funzionale, functional food, definizione dell'americana Institute of Medicine's Food and Nutrition Board che comprende ogni cibo o ingrediente di cibo che può determinare un beneficio sulla salute attraverso i nutrienti contenuti. ${ }^{14}$

Secondo la definizione più rigorosa del Canadian Bureau of Nutritional Sciences of Food Directorate of 
Health i nutraceutici sono prodotti isolati o purificati del cibo, che generalmente sono in forma di medicinali non usualmente associati al cibo. È dimostrato che inducano un beneficio fisiologico o possiedano un effetto protettivo nei confronti delle malattie croniche. ${ }^{15}$ Il cibo funzionale invece, è simile in apparenza o può essere un cibo convenzionale, viene consumato come parte di una dieta abituale, di esso è stato dimostrato un beneficio fisiologico e/o la riduzione del rischio di malattia cronica.

La Food and Drug Administration (FDA) e la European Food Safety Authority (EFSA) regolano la commercializzazione di supplementi dietetici e nutraceutici al fine di garantirne la sicurezza.

A causa del largo numero di componenti è difficile classificare tutti i nutraceutici. La classificazione più diffusa suddivide i nutraceutici in tradizionali e non tradizionali, a seconda che vi sia o meno l'intervento dell'uomo nell'aggiungere nutrienti ai cibi stessi (Tabella 1). ${ }^{16}$

Per la descrizione dettagliata dei prodotti disponibili in commercio è possibile consultare online il sito http://www.aboutbioscience.org.

In Italia, nel 2009, è nata la Società Italiana di Nutraceutica (SINut) con lo scopo di promuovere ricerche e studi in ambito nutraceutico.

\section{Nutraceutici e dislipidemia: ruolo dei nutraceutici nel trattamento dell'ipercolesterolemia}

La letteratura riporta più di 40 nutraceutici con supposto effetto sul metabolismo lipidico, ${ }^{17}$ alcune di queste sostanze hanno una provata efficacia nel ridurre sia i livelli di lipidi sierici sia il rischio cardiovascolare, in aggiunta alcuni hanno dimostrato efficacia su markers surrogarti di danno vascolare. Tuttavia molti studi presentano limiti di disegno dello studio, di selezione di popolazione e di indici di outcome.

\section{Soia}

Le conoscenze sui prodotti naturali spesso sono originate da studi osservazionali di popolazione, ad esempio è stato ipotizzato che il minor rischio cardiovascolare della popolazione asiatica fosse legato al consumo di soia.

La soia è un'eccellente fonte di proteine e contiene gli isoflavoni, sostanze micronutrienti, strutturalmente simili al $17-\beta$ estradiolo in grado di legare $i$ recettori A e B dell'estrogeno come agonisti incompleti. Gli isoflavoni hanno dunque proprietà simili agli estrogeni, incluso l'effetto di riduzione dei livelli di colesterolo e l'inibizione dell'ossidazione di LDL, ad essi si attribuiscono i benefici indotti dalla soia.

Gli studi clinici effettuati disponibili utilizzano prodotti con contenuto differente di soia. In una metanalisi di 29 trials è stato dimostrato che il consumo di $47 \mathrm{~g} /$ die di proteine della soia riduce il colesterolo totale del $9 \%$, il colesterolo LDL del $12,9 \%$ e i trigliceridi del $10,5 \%$ senza un effetto significativo sul colesterolo con lipoproteine ad alta densità (HDL). ${ }^{18} \mathrm{La}$ revisione di 22 trials randomizzati ad opera dell' American Heart Association (AHA) nel 2006 ha evidenziato però solo la riduzione del 3\% di LDL senza effetti su HDL, trigliceridi e pressione sanguigna nonostante il contenuto di $50 \mathrm{~g} /$ die di proteine di soia.

Tabella 1. Classificazione nutraceutici.

\begin{tabular}{|c|c|c|c|}
\hline \multirow[t]{7}{*}{ Tradizionali } & \multirow[t]{3}{*}{ Costituenti chimici } & Nutrienti & Es. vitamine, aminoacidi, sali minerali, acidi grassi \\
\hline & & Erbe & $\begin{array}{l}\text { Willow bark (Salix nigra), Parsley (Petroselinum } \\
\text { cripsum), Peppermint (Mentha piperita), Lavandula } \\
\text { angustifolia), Cranberries (Vaccinium erythrocarpum) }\end{array}$ \\
\hline & & Fitochimici & $\begin{array}{l}\text { Es. carotenoidi, polifenoli flavonoidi e non flavonidi, } \\
\text { acidi fenolici }\end{array}$ \\
\hline & $\begin{array}{l}\text { Probiotici } \\
\text { Microorganismi che conferiscono } \\
\text { un beneficio all'ospite }\end{array}$ & & $\begin{array}{l}\text { Es. Lactobacillus } \\
\text { Bifido-bacterium }\end{array}$ \\
\hline & \multirow[t]{3}{*}{ Enzimi } & Derivati da piante & Pectinase \\
\hline & & Derivati da agenti microbici & $\begin{array}{l}\text { Hemicellulase } \\
\text { Catalase }\end{array}$ \\
\hline & & Derivati da animali & Lysozyme \\
\hline \multirow[t]{2}{*}{ Non tradizionali } & $\begin{array}{l}\text { Fortificati, con aggiunta } \\
\text { di nutrienti e/o ingredienti }\end{array}$ & & \\
\hline & $\begin{array}{l}\text { Ricombinanti } \\
\text { Risultato di processi di bioingegneria }\end{array}$ & & \\
\hline
\end{tabular}

Modificata da Singh e Sinha, 2012. ${ }^{16}$ 
Alcune evidenze inoltre hanno attribuito un ruolo marginale agli isoflavoni nella riduzione del colesterolo per cui non ne viene raccomandata l'assunzione dalla AHA. In letteratura sono pubblicati anche studi in cui si segnala l'aumento della pressione arteriosa e del livello di lipidi legata all'assunzione di soia. Da ultimo non è ancora completamente chiarito se l'attività estrogeno-like degli isoflavoni della soia incrementi il rischio di neoplasia mammaria. ${ }^{19}$

La Nutrition Commitee of AHA sostiene che l'utilizzo sostitutivo delle proteine di soia non appare avere un beneficio clinico, così come la supplementazione con gli isoflavoni non dovrebbe avere l'obiettivo di migliorare l'assetto lipidico e il rischio cardiovascolare. ${ }^{20}$ Gli esperti concludono che i prodotti contenenti soia e derivati (tofu, burro di soia, edamame) utilizzati in sostituzione di cibi a contenuto proteico animale, determinano un beneficio per il loro contenuto in acidi grassi polinsaturi, fibre, vitamine e minerali e per il basso contenuto di grassi saturi.

\section{Le fibre}

Le fibre sono classificate a seconda della solubilità in acqua in insolubili come lignina, cellulosa e alcune emicellulose e solubili come la pectina e la mucillagine. Più di 50 anni fa Keys osservò come alcuni tipi di diete contenenti fibre riducessero il livello di colesterolo. ${ }^{21}$ Il meccanismo d'azione delle fibre non è chiaro ma si suppone del sequestro del colesterolo tramite gli acidi biliari, dalla up-regolazione dei recettori epatici di LDL, dalla inibizione della sintesi di grassi a livello epatico, della riduzione dell'assorbimento di macronutrienti a livello intestinale e dell'azione sulla motilità intestinale e sulla sensibilità all'insulina, da ultimo le fibre inducono sazietà. La reale efficacia sulla riduzione del colesterolo indotta da una dieta a base di fibre è materia di dibattito a causa della mancata omogeneità degli studi condotti. Nella revisione di Brown di 67 studi controllati che hanno riguardato circa 3000 individui si conclude che l'introduzione giornaliera da $2 \mathrm{a} 10 \mathrm{~g}$ di fibre solubili riduce il colesterolo totale di $1,75 \mathrm{mg} / \mathrm{dL}$ per ogni grammo di fibre e riduce il colesterolo LDL di $2,2 \mathrm{mg} / \mathrm{dL}$ senza differenze tra i tipi di fibre solubili. ${ }^{22}$ Molti studi sostengono la teoria che le fibre solubili siano in grado di ridurre il colesterolo LDL, mente le fibre insolubili agirebbero solo sulla motilità intestinale. ${ }^{23,24}$ Fibre solubili concentrate derivate dalle alghe come lo psyllium, nori e kombu sono disponibili in diversi prodotti, in una metanalisi si evince che il consumo di $10 \mathrm{~g} / \mathrm{die}$ ridurrebbe il colesterolo totale del $4 \%$ e il colesterolo LDL del $7 \%$.

In riferimento alla riduzione del rischio cardiovascolare in 11 studi prospettici è stato dimostrato come $10 \mathrm{~g}$ di fibre/die siano associate alla riduzione del rischio di eventi coronarici del $14 \%$ e di morte corona- rica del $27 \% .{ }^{25}$ Lo studio PREDIMED (prevenzione con dieta mediterranea) ha rilevato la riduzione di peso, circonferenza addominale, pressione, glucosio e incremento di colesterolo HDL in una coorte di pazienti ad alto rischio cardiovascolare (diabetici + almeno 3 fattori di rischio cardiovascolare) che assumevano una dieta ad alto contenuto di fibre. ${ }^{26}$

Basandosi sui dati cumulativi degli studi, la società americana US dietary reference intakes raccomanda l'assunzione di $38 \mathrm{~g} /$ die di fibre per gli uomini e 25 $\mathrm{g} /$ die per le donne. ${ }^{27}$

L'assunzione dietetica di fibre può essere aumentata con un maggior consumo quotidiano di vegetali (specie verdure, la frutta contiene a parità di peso meno fibre), ma anche sostituendo le farine raffinate con pane, pasta e riso integrali e/o utilizzando alimenti funzionali arricchiti in fibre (usualmente viene impiegato $\beta$-glucano, derivato dall'avena). In commercio sono disponibili alcuni cibi funzionali arricchiti di fibre solubili e insolubili come succhi, yogurt e barrette di cereali. Le fibre possono essere anche assunte come supplemento: la fibra supplementata più studiata è indubbiamente lo psillio micronizzato, derivato dalla Plantago ovata. Di grande interesse sono anche le proprietà del glucomannano e del fieno greco: il loro effetto ipocolesterolemizzante è relativamente dosedipendente e variabile da individuo a individuo, stimabile tra il 5 e il $10 \%$.

Inoltre anche una terapia di combinazione con fibre solubili e steroli vegetabili può indurre un beneficio. In uno studio randomizzato in pazienti con colesterolo LDL tra 100 e 160 dopo 4 settimane di terapia, l'utilizzo di $2,6 \mathrm{~g} / \mathrm{die}$ di steroli vegetali e 10 $\mathrm{g} /$ die di psyllium ha permesso di ottenenere il decremento del $10 \%$ di colesterolo LDL. ${ }^{28}$

Accanto ai risultati ottenuti sui livelli lipidici, da segnalare uno studio osservazione condotto su popolazione mediterranea ad alto rischio cardiovascolare, in cui è stata evidenziata la correlazione tra incremento dell'introito di fibre e riduzione dello spessore medio intimale carotideo. ${ }^{29}$

\section{$\beta$-glucani}

I $\beta$-glucani (1-3,1-4 $\beta$-D-glucani) sono polisaccaridi del glucosio, in cui il legame chimico tra le singole molecole del glucosio li rende non digeribili dagli enzimi amidolitici intestinati. Il $\beta$-glucano è quindi, a tutti gli effetti, una fibra alimentare, presente nella crusca dei chicchi dei cereali, in particolare di orzo e avena, meno nella segale e nel frumento, altre fonti sono il lievito del pane, alcuni miceti e alcuni tipi di funghi. A contatto con l'acqua, i $\beta$-glucani formano una massa viscosa, in grado di svolgere più funzioni benefiche, sia sul transito intestinale, sia sul metabolismo lipidico e glucidico. La dose quotidiana di almeno $3 \mathrm{~g}$, inserita in un'alimentazione comunque equilibrata, contribuisce a ridurre la 
colesterolemia del 5-12\%. Inoltre grazie alla loro viscosità, i $\beta$-glucani anche in quantità limitate, ritardano lo svuotamento gastrico e quindi l'assorbimento di glucosio.

\section{Steroli vegetali e stanoli}

I fitosteroli ( $\beta$-sitosterolo, campesterolo, avenasterolo, ecc.) sono presenti ad alte concentrazioni in molti semi e negli oli che ne derivano, specie nell'olio di mais, nella frutta secca e in molte spezie. I fitosteroli, estratti e concentrati, possono inoltre essere addizionati ai cosiddetti alimenti funzionali: yogurt, bevande a base di latte fermentato, margarine e prodotti da forno.

L'effetto ipocolesterolemizzante di steroli e stanoli vegetali è noto da decenni. ${ }^{30}$ Entrambe queste classi di molecole posseggono una struttura chimica molto simile a quella del colesterolo; essi, per questa analogia strutturale competono con il colesterolo alimentare o di origine biliare presente nel lume intestinale, limitandone l'assorbimento $(-30 / 40 \%)$ e riducendo così significativamente la colesterolemia. Una recente meta-analisi di 124 studi clinici controllati mostra, infatti, come la supplementazione quotidiana di fitosteroli, in dosi comprese tra 0,6 e 3 g, porti a una riduzione dose-dipendente della colesterolemia LDL, fino al $12 \% .{ }^{31}$ Dosi maggiori non sembrano apportare vantaggi significativi; secondo alcuni autori, anzi, aumenterebbe il rischio di assorbimento intestinale dei fitosteroli stessi. I prodotti arricchiti in fitosteroli dovrebbero essere consumati al termine del pasto principale ed assunti con regolarità, di fatto cronicamente; all'interruzione del consumo fa seguito un'immediata risalita della colesterolemia.

Un numero di prodotti contenenti gli steroli vegetali sono disponibili, ma la limitazione nello sviluppo è legata alla scarsa solubilità. La soluzione trovata risiede nella loro esterificazione con acidi grassi per permetterne la disponibilità in cibi quali margarina $\mathrm{e}$ condimenti. L'introduzione di 0,8 fino a $3 \mathrm{~g} /$ die di stanoli e/o steroli nelle margarine permette di abbassare i livelli di colesterolo.

Diversi studi hanno valutato le risposte di efficacia. In uno studio randomizzato che ha coinvolto 153 persone con ipercolesterolemia lieve il consumo di margarina arricchita di sitostanolo permetteva la riduzione di LDL del 10-14\%, prevalentemente nei primi 3 mesi, sebbene la riduzione continuasse per 12 mesi, senza differenze significative per HDL e trigliceridi, inoltre non era inficiato l'assorbimento di vitamine liposolubili sebbene i livelli di $\beta$ carotene risultassero più bassi.

I dati sono stati confermati anche in piccoli studi su donne in post menopausa con malattia coronarica e in 10 donne in terapia con simvastatina dove l'utilizzo di margarina arricchita permetteva di ridurre il fabbisogno di statine.
Altri studi evidenziano come la margarina arricchita da stanoli induca la riduzione di colesterolo anche in pazienti che ricevono una dose stabile di statine. Ad esempio in una serie di 167 pazienti con LDL $\geq \mathrm{a} 130 \mathrm{mg} / \mathrm{die}$ nonostante 3 mesi di statine, $5,1 \mathrm{~g} / \mathrm{die}$ di stanoli vegetali per 8 settimane determinavano la riduzione del colesterolo totale del $12 \%$ e colesterolo LDL (c-LDL) del 17\%.

In questi studi, seppur limitati, non sono stati evidenziati eventi avversi nell'utilizzo di margarina arricchita con esteri stanolici e steroli delle piante, risulta però possibile che sul lungo termine riducano la concentrazione plasmatica di antiossidanti come il $\beta$-carotene e l' $\alpha$-tocoferolo.

A oggi non esistono però studi che attribuiscano agli esteri stanolici contenuti nella margarina un effetto di riduzione di incidenza di eventi coronarici.

Un altro modo per permettere l'assunzione di stanoli vegetali senza ingerire grassi è quello di creare un'emulsione con la lecitina che può essere formulata come uno spray secco polverizzato. Sono disponibili in commercio in formato di tavolette contenenti 1,3$1,9 \mathrm{~g}$ di stanoli vegetali. Sono disponibili anche dati di steroli vegetali combinati con il cioccolato nero.

La Nutrition Committee of AHA pur riconoscendo agli alimenti contenenti steroli vegetali/esteri stanolici un promettente ruolo per le diete, questi prodotti non possono essere utilizzati di routine nella popolazione generale fino a quando non vengano effettuati studi di sicurezza sul lungo termine, al momento il loro uso deve essere limitato alla popolazione adulta con ipercolesterolemia o con eventi aterosclerotici che richiedano una riduzione di LDL. Sebbene possano essere utilizzati nei bambini, i livelli di lipoproteine liposolubili devono essere attentamente monitorati. ${ }^{31}$

\section{Policosanoli}

I policosanoli sono una miscela di polialcoli di diversa lunghezza. Si estraggono dalla cera della canna da zucchero, dalla cera d'api, dalla crusca di riso e da altri vegetali quali le patate. Risultano ben tollerati e alcuni studi suggeriscono che la dose di 10-20 mg/die sia in grado di ridurre la concentrazione di LDL del $25 \%$ o più e potrebbe innalzare i valori di HDL fino al $15 \% .{ }^{32}$ Tuttavia un trial multicentrico randomizzato su 143 pazienti non ha evidenziato differenze significative con range di dosaggio da 10 a $80 \mathrm{mg} / \mathrm{die} \cdot{ }^{33} \mathrm{I}$ pochi dati di endpoints clinici disponibili, il meccanismo d'azione non completamente chiarito e la variabilità nelle preparazioni dei prodotti rendono il loro utilizzo poco raccomandato da molti autori.

\section{Riso rosso fermentato}

Il riso rosso fermentato è ottenuto dalla fermentazione del comune riso da cucina (Oryza sativa) ad 
opera di un particolare lievito, il Monascus purpureus o lievito rosso; durante la sua attività fermentatrice, si arricchisce di un gruppo di 14 sostanze, denominate monacoline a cui è stata attribuita scientificamente una spiccata attività ipocolesterolemizzante. Tra esse, la monacolina K riproduce la stuttura chimica e l'azione farmacologica della lovastatina, risultando in grado di inibire la HMG-CoA reduttasi, enzima chiave nella biosintesi del colesterolo. L'efficacia del riso rosso fermentato nel ridurre il colesterolo è stata valutata in uno studio prospettico in doppio cieco in cui 83 pazienti con iperlipidemia che non ricevevano terapia specifica erano randomizzati ad assumere riso rosso fermentato $(2,4 \mathrm{~g} / \mathrm{die})$ o placebo, entrambi i gruppi dovevano seguire una dieta a basso contenuto di colesterolo. ${ }^{34} \mathrm{E}$ stato ottenuto, dopo otto settimane, un decremento significativo del colesterolo totale rispetto al valore basale nei pazienti trattati rispetto al gruppo di controllo, in particolare si riduceva significativamente la concentrazione di c-LDL, senza effetto su HDL. Altri studi hanno confermato quest'osservazione attribuendo al riso rosso fermentato la capacità di ridurre il colesterolo totale e LDL. ${ }^{35,36} \mathrm{Un}$ recente studio su modello murino ha rilevato un'interessante riduzione non solo dei livelli circolanti di colesterolo, ma anche dell'accumulo di colesterolo a livello epatico e della conseguente steatosi. ${ }^{37}$

L'assunzione quotidiana della lovastatina contenuta nel riso rosso fermentato è lo $0,2 \%$ del prodotto, dunque l'assunzione di 2,4 g/die di riso rosso fermentato apporta una dose di lovastatina pari a $4,8 \mathrm{mg}$, dosaggio considerevolmente minore rispetto alle somministrazioni di 20$40 \mathrm{mg}$ usati nei trials clinici, questo suggerisce che vi siano altri ingredienti attivi che contribuiscono alla riduzione del colesterolo come gli steroli ( $\beta$-sitosterolo, campesterolo, stigma sterolo, sapogenina) gli isoflavoni e gli acidi grassi monoinsaturi. ${ }^{38}$

L'uso del riso rosso fermentato cinese è aumentato esponenzialmente negli ultimi anni per l'interesse pubblico occidentale per le medicine alternative e grazie alla pubblicazione di studi controllati e randomizzati che ne dimostravano l'efficacia. Tale prodotto si pone in alternativa alla terapia farmacologica ipolipemizzante in quei pazienti che non vogliono assumere statine per ragioni filosofiche o che presentano intolleranza epatica o muscolare alle statine.

In un trial randomizzato, pazienti che avevano interrotto le statine per mialgia, l'utilizzo di riso rosso fermentato $1800 \mathrm{mg} \times 2 /$ die $v s$ placebo determinava una significativa riduzione di colesterolo LDL. ${ }^{36}$ Tuttavia non vi sono studi di confronto con la statina nel braccio di controllo.

Da segnalare come non tutti i preparati di riso rosso fermentato inducono lo stesso risultato, questo dipende dalla sostanziale variabilità tra i prodotti in commercio, in particolare per le concentrazioni di mo- nacolina $\mathrm{K}(0,10$ a $10 \mathrm{mg} / \mathrm{capsula})$, inoltre alcuni preparati hanno un elevato livello di citrinina, una micotossina nefrotossica. Per questo, mentre studi sul breve termine (fino a quattro mesi) hanno trovato il riso rosso sicuro, non sono stati condotti studi a lungo termine. In USA il commercio del riso rosso fermentato è proibito.

\section{Polifenoli}

I polifenoli sono sostanze che si trovano principalmente nelle piante e nei cibi derivati da piante come the, caffè, cacao, olio d'oliva, vino rosso, risultano avere proprietà antiossidanti, immunomodulatorie, vasodilatatorie che possono contribuire alla riduzione del rischio cardiovascolare. I polifenoli comprendono i flavonoidi e i flavonidi derivati, acidi fenolici e lo stilbene. Uno studio osservazionale ha evidenziato un'inversa associazione tra mortalità e assunzione di flava noni. ${ }^{39}$

Uno studio randomizzato su 200 maschi ha comparato l'effetto sui lipidi sierici dell'olio extra-vergine d'oliva, ad elevato contenuto di polifenoli, rispetto all'olio d'oliva raffinato, a basso contenuto in polifenoli, e di un misto dei 2 oli. È stata evidenziata una risposta dose dipendente, dove l'effetto dell'olio d'oliva era maggiore rispetto agli altri su HDL e LDL.

Al resveratrolo, un polifenolo presente in numerose piante, sono stati attribuiti diversi benefici, in particolare contro l'aterosclerosi.

Nel vino rosso si trova la maggiore concentrazione di resveratrolo $(0,847 \mathrm{mg} / 100 \mathrm{~g})$ e ad esso è stato attribuito erroneamente il beneficio sul metabolismo del colesterolo, definito anche paradosso francese.

In un lavoro su modelli murini del 2012, Chen avrebbe evidenziato la capacità del resveratrolo di incrementare la trascrizione di m-RNA della $7 \alpha$-idrossilasi (CYP7A1), enzima coinvolto nel metabolismo del colesterolo. ${ }^{40}$ Tuttavia una recente metanalisi non ha confermato il ruolo del resveratrolo nella dislipidemia umana, questo potrebbe essere dovuto al metabolismo di primo passaggio del resveratrolo nell'uomo che risulta dunque attivo solo in una piccola porzione. Da questi studi è derivata la conclusione che il resveratrolo eserciti una funzione positiva down-regolando le proteine proinfiammatorie o inibendo l'ossidazione del colesterolo LDL. ${ }^{41}$

I polifenoli contenuti nel gelso appaiono corroborare i risultati riguardo al resveratrolo, sono efficaci nel ridurre i depositi di acidi grassi epatici e interferiscono con gli enzimi coinvolti nel metabolismo lipidico, come la sintetasi degli acidi grassi, dell'acetil CoA carbossilasi o della 3-idro3 metilglutarylCoa reduttasi. ${ }^{42}$ La teaflavina, il maggior polifenolo contenuto nel the nero, ha dimostrato avere un effetto di riduzione dei lipidi. ${ }^{43}$ Altri studi hanno evidenziato come bevande funzionali a base di frutta ricche in polifenoli siano in grado di ridurre i livelli di colesterolo 
epatico e del colesterolo totale ma non di HDL in modelli murini ipertesi con dieta ricca di colesterolo.

Tra i polifenoli attivi contenuti nell'olio extravergine di oliva di particolare interesse risulta essere l'idrossitirosolo, una molecola di piccole dimensioni con buona biodisponibilità e ottimo assorbimento nel tratto gastrointestinale. L'idrossitirosolo è dotato di elevato potere antiossidante come risulta nella scala ORAC (oxygen radicals absorbance capacity). Nel 2011 1'EFSA (European Food and Safety Authority) ne ha riconosciuto il ruolo inibitorio dell'ossidazione del colesterolo LDL. ${ }^{44}$

In uno studio randomizzato su volontari sani è stato dimostrato il ruolo dell'olio d'oliva ricco in polifenoli nel ridurre non solo l'ossidazione del colesterolo LDL ma anche l'espressione del ligando del CD40 e dei geni coinvolti nei processi infiammatori e di aterogenesi. ${ }^{45}$

\section{Noci}

Piccoli studi randomizzati hanno dimostrato che le noci, che sono ricche di acidi grassi polinsaturi, svolgono un ruolo positivo sull'assetto lipidico. Ad esempio, un trial che ha comparato la dieta mediterranea con una dieta in cui le noci costituivano il 35\% dell'apporto energetico ottenuto da grassi monoinsaturi, ha rilevato una riduzione del $4 \%$ di colesterolo totale e del 6\% di c-LDL.

Altri trials hanno dimostrato riduzioni simili dei lipidi utilizzando mandorle, pistacchi e altre nocciole. Da un'analisi degli studi emerge come un introito medio giornaliero di $67 \mathrm{~g}$ di nocciole, sia in grado di ridurre il colesterolo totale di $10,9 \mathrm{mg} / \mathrm{dL}$ mentre il colesterolo LDL aumenta di 10,2 mg/dL. ${ }^{46}$ È stato evidenziato un effetto dose-risposta sui livelli lipidici, dato che è stato confermato anche in studi che hanno previsto l'assunzione di differenti tipi di nocciole.

Nel trial PREDIMED, l'introduzione di $30 \mathrm{~g}$ di noci (15 $\mathrm{g}$ di noci, 7,5 di nocciole, 7,5 $\mathrm{g}$ di mandorle) in una dieta mediterranea, ha permesso di ridurre $\mathrm{i}$ livelli di colesterolo LDL e trigliceridi. ${ }^{47}$

Vi sono inoltre evidenze che l'incrementato introito di noci, 4 volte la settimana, è associato con un miglioramento dell'outcome cardiovascolare, con riduzione del tasso di mortalità per malattia coronarica. ${ }^{48}$

\section{Guggulipid}

È un preparato standardizzato di guggul, un estratto di resina di una pianta nativa dell'India (Commiphora mukul) usata nella medicina tradizionale ayurvedica per il trattamento dell'epilessia, ulcera, obesità, artrite reumatoide e aterosclerosi.

Guggulipid contiene Z- and E-guggulsteroni, che sono la parte attiva e hanno dimostrato efficacia nel- l'inibire la sintesi del colesterolo a livello epatico antagonizzandone il recettore.

A carico del guggul è stata dimostrata inoltre un'attività sequestrante i lipidi a livello intestinale.

Tuttavia uno studio randomizzato condotto negli USA, che ha coinvolto 103 pazienti con ipercolesterolemia, ha evidenziato un incremento significativo di LDL e l'insorgenza di rash cutaneo. ${ }^{49}$ Molti autori non ne consigliano l'utilizzo.

\section{Berberina}

La berberina è un alcaloide naturale, presente in notevoli concentrazioni in numerose piante medicinali (Hydrastis canadensis, Coptis chinensis, Berberis aquafolium, Berberis vulgaris, Berberis aristata). L'azione si esplica a livello epatico, ma in modo complesso e non ancora completamente chiarito, probabilmente molto vicino a quello degli ipocolesterolemizzanti più recenti, gli antagonisti dell'enzima PCSK9. Gli studi dimostrano che, alla dose di 500 $\mathrm{mg} / \mathrm{die}$, si ottiene una riduzione sia della colesterolemia LDL (-20\%) sia della trigliceridemia (-25\%).

\section{Selenio}

Studi osservazionali hanno evidenziato un legame tra l'incremento del selenio e colesterolo ma studi randomizzati non hanno trovato un beneficio tra supplementazione e outcome cardiovascolare, per cui l'assunzione con questa finalità non è raccomandata. ${ }^{50}$

\section{Altre sostanze}

Di altri fitoterapici a cui si fa ricorso per la riduzione della colesterolemia, tra cui gli estratti di aglio, carciofo e cardo mariano, non è ancora noto il principio attivo responsabile.

È stato condotto un largo studio che randomizzava, verso placebo, l'assunzione di tre differenti preparati di aglio (naturale, in polvere o estratto) equivalenti a uno spicchio di 4 grammi per 6 giorni la settimana per 6 mesi. Non sono state evidenziate riduzioni significative dei livelli di colesterolo. ${ }^{51}$

Un'altra sostanza di interesse è il $\gamma$-orizanolo, estratto dalla crusca del riso, che agirebbe interferendo con l'assorbimento intestinale del colesterolo presente negli alimenti. Infine, da citare sono i flavoni metossilati presenti nella buccia degli agrumi: impiegati come estratto, sembrano inibire in modo diretto la formazione sia di LDL sia di lipoproteine a densità molto bassa. Le dimostrazioni di efficacia nell'uomo sono però ancora carenti.

\section{Compositi}

In Italia la maggior parte dei nutraceutici ad azione ipolipemizzante si trova in commercio sotto forma di 
Tabella 2. Conclusioni sull'uso dei nutraceutici.

L'approccio non farmacologico rappresenta il primo step di cura nell'ipercolestrolemia

Le statine e altri farmaci spesso falliscono nell'obiettivo di ridurre i livelli sierici di lipidi

I nutraceutici possono agire positivamente sulla displipidemia

Numerosi nutraceutici si sono dimostrati efficaci nell'influenzare la concentrazione sierica di colesterolo con pochi effetti collaterali

Il riso rosso fermentato e gli steroli/stanoli vegetali si sono dimostrati i più efficaci

L'uso dei nutraceutici dovrebbe essere considerato nei pazienti ipercolesterolemici in cui l'uso delle statine non è indicato, esempio nei pazienti con basso profilo di rischio cardiovascolare

Necessitano di essere ulteriormente individuati in futuri studi: l'indicazione all'inizio del trattamento con nutraceutici, l'influenza su end-points cardiovascolari, la sicurezza e la realizzabilità dei processi di produzione

composito, ovvero di associazione di diverse sostanze, a dosaggi che assicurino l'efficacia, senza compromettere la tollerabilità. Tra le associazioni più testate vi è quella tra monacoline (3 mg) e policosanoli (10 mg), che porterebbe a una riduzione della colesterolemia LDL di circa il 20\%. Un'altra associazione nota è quella tra policosanoli, che inibiscono la sintesi del colesterolo e berberina, che facilita l'eliminazione del colesterolo circolante: in questo modo si raggiunge una riduzione della colesterolemia fino al 25\%, ma anche una riduzione della trigliceridemia fino al 20\%, risultando efficaci anche in soggetti che non possono assumere statine e con un effetto dimostrato sulla vasodilatazione e sull'onda di pulsazione aortica, noti marcatori indiretti di salute vascolare.

Sono stati effettuati alcuni studi di confronto tra nutraceutici e statine. Nel 2013 Ruscica ha pubblicato il risultato di un piccolo trial che aveva previsto l'arruolamento di 30 soggetti con displipidemia moderata e sindrome metabolica randomizzati a ricevere per 8 settimane una combinazione di nutraceutici (Armolipid plus $^{\mathbb{R}}$ ) oppure pravastatina $10 \mathrm{mg} / \mathrm{die}$. Lo studio evidenziava come i nutraceutici permettessero di ottenere una riduzione del $12,8 \%$ di colesterolo totale e del $21,1 \%$ di LDL in modo sovrapponibile a quanto ottenuto dalla statina. L'autore conclude che tale approccio, che risulta sicuro ed efficace, può essere utile nel trattamento di pazienti a rischio cardiovascolare moderato in cui l'approccio farmacologico non risulti appropriato. ${ }^{52}$

\section{Conclusioni}

Il controllo della colesterolemia è da sempre un tema complesso, nel quale confluiscono più componenti.

I nutraceutici rappresentano una categoria di alimenti emergenti, che possono apportare benefici sulla salute attraverso elementi nutrizionali. In Tabella 2 vengono sintetizzati le conclusioni sull'uso dei nutraceutici. Numerosi aspetti devono comunque ancora essere definiti, la loro efficacia è spesso limitata dalle dosi insufficienti legate alla somministrazione orale.
Sono fiorenti gli studi di bioingegneria al fine di migliorare la formulazione e la biodisponibilità dei nutraceutici. La supplementazione della dieta con i nutraceutici ha dimostrato diversi livelli di efficacia nel modificare il profilo lipidico. Mentre i risultati per i derivati della soia, per le fibre e i policosanoli sono contrastanti, gli effeti dei fitosteroli e del riso rosso fermentato da solo o in combinazione con altri nutraceutici appiano essere convincenti. I dati disponibili evidenziano una riduzione del 10-20\% del livello di colesterolo in soggetti con ipercolesterolemia poligenica, ipercolesterolemia familiare eterozigote, con miopatia da statine e nei ragazzi.

La sicurezza dei nutraceutici è stata valutata su campioni numerosi di individui iperlipidemici.

Restano tuttavia da chiarire alcuni aspetti, quali l'effetto dell'abbassamento del colesterolo nel tempo, il loro reale impatto su endpoint cardiovascolari considerati hard e la realizzabilità dei processi produttivi.

Sebbene gli standard della evidence-based medicine non permettono al momento di trarre conclusioni definitive sull'uso nella pratica clinica, risulta condivisibile il pensiero della necessità di individuare specifiche categorie di individui suscettibili di un beneficio derivante dall'utilizzo dei nutraceutici. ${ }^{9}$

\section{Bibliografia}

1. Cziraky MJ, Tan H, Bullano MF, et al. Impact of optimal lipid value achievement between 2005 and 2009 in patients with mixed dyslipidaemia on cardiovascular event rates. Int J Clin Pract 2012;65:425-35.

2. Martin SS, Gosch K, Kulkarni KR, et al. Modifiable factors associated with failure to attain low-density lipoprotein cholesterol goal at 6 months after acute myocardial infarction. Am Heart J 2013;165:26-33.

3. Shay CM, Ning H, Allen NB, et al. Status of cardiovascular health in US adults: prevalence estimates from the National Health and Nutrition Surveys (NHANES) 2003-2008. Circulation 2012;125:45-56.

4. Arca M, Pigna G. Treating statin-intolerant patients. Diabetes Metab Syndr Obes 2011;4:155-66. 
5. Abd TT, Jacobson TA. Statin-induced myopathy: a review and update. Expert Opin Drug Saf 2011;10:373-87.

6. Blaha MJ, Nasir K, Blumenthal RS. Statin therapy for healthy men identified as "increased risk". JAMA 2012; 307:1489-90.

7. Redberg RF, Katz MH. Healthy men should not take statins. JAMA 2012;307:1491-2.

8. Stone NJ, Robinson J, Lichtenstein AH, et al. 2013 ACC/AHA Guideline on the Treatment of Blood Cholesterol to Reduce Atherosclerotic Cardiovascular Risk in Adults: a report of the American College of Cardiology/American Heart Association Task Force on Practice Guidelines. Circulation 2014;129:S1-45.

9. Mannarino M, Ministrini S, Pirro M. Nutraceuticals for the treatment of hypercholesterolemia. Eur J Int Med 2014;25:592-9.

10. Scicchitano P, Cameli M, Maiello M, et al. Nutraceticals and dyslipidemia:beyond the common therapeutics. J Funct Food 2014;6:11-32.

11. Marazzi G, Cacciotti L, Pelliccia F, et al. Long-term effects of nutraceuticals (berberine, red yeast rice, policosanol) in elderly hypercholesterolemic patients. Adv Ther 2011;28:1105-13.

12. Sirtori CR, Galli C, Anderson JW, et al. Nutritional and nutraceutical approaches to dyslipidemia and atherosclerosis prevention: focus on dietary proteins. Atherosclerosis 2009;203:8-17.

13. DeFelice SL. The nutraceutical revolution: its impact on food industry R\&D. Trends Food Sci Technol 1995;6: 59-61.

14. Ross S. Functional foods: the Food and Drug Administration perspective. Am J Clin Nutr 2000;71:1735S-8S.

15. Health Canada. Policy paper: nutraceuticals/functional foods and health claims on foods. Government of Canada: Therapeutic products program and the food directorate from the health protection branch. November 2, 1998. [cited Jan 8, 2008]. Available from http://www.hc-sc.gc.ca

16. Singh J, Sinha S. Classification, regulatory acts and applications of nutraceuticals for health. Int J Pharma Biosci 2012;2:177-87.

17. Houston M. The role of nutraceutical supplements in the treatment of dyslipidemia. J Clin Hypertens 2012;14: 121-32.

18. Anderson JW, Johnstone BM, Cook-Newell ME. Metaanalysis of the effects of soy protein on serum lipids. $\mathrm{N}$ Engl J Med 1995;333:276-82.

19. Teede HJ, Dalais FS, Kotsopoulos D, et al. Dietary soy has both beneficial and potentially adverse cardiovascular effects: a placebo-controlled study in men and postmenopausal women. J Clin Endocrinol Metab 2001;86: 3053.

20. Sacks FM, Lichtenstein A, Van Horn L, et al. Soy protein, isoflavones, and cardiovascular heart: an American Hearth Association Science Advisory for professionals fron Nutrition Committee. Circulation 2006;113:1034.

21. Keys A, Grande F, Anderson JT. Fibre and pectin in the diet and serum cholesterol concentration in man. Proc Soc Exp Biol 1961;106:555-8.

22. Glore SR, Van Treeck D, Knehans AW, Guild M. Soluble fiber and serum lipids: a literature review. J Am Diet Assoc 1994;94:425-36.

23. Kris-Etherton PM, Krummel D, Russell ME, et al. The effect of diet on plasma lipids, lipoproteins, and coronary heart disease. J Am Diet Assoc 1988;88:1373-400.
24. PereiraMA, O'Reilly E, Augustsson K, et al. Dietary fiber and risk of coronary heart disease: a pooled analysis of cohort studies. Arch Intern Med 2004;164:370-6.

25. Estruch R, Martinez-Gonzalez MA, Corella D, et al. Effects of dietary fibre intake on risk factors for cardiovascular disease in subjects at high risk. J Epidemiol Community Health 2009;63:582-8.

26. National Research Council. Dietary reference intakes for energy, carbohydrate, fiber, fat, fatty acids, cholesterol, protein, and amino acids (macronutrients). Washington, DC: The National Academies Press; 2005.

27. Sherestha S, Volek JS, Udani J, et al. A combination therapy including psyllium and plant sterols lowers LDL cholesterol by modifyin lipoprotein metabolism in hyercholesterolemic individuals. J Nutr 2006;136:2492.

28. Buil-Cosiales P, Irima P, Ros E, et al. Dietary fibre intake in inversely associated with carotid intim-media thickness: a cross sectional assessment in the PREDIMED study. Eur J Clin Nutr 2009;63:1213.

29. Katan MB, Grundy SM, Jones P, et al. Efficacy and safety of plant stanols and sterols in the management of blood cholesterol levels. Mayo Clin Proc 2003;78:965-78.

30. Amir Shaghaghi M, Abumweis SS, Jones PJ. Cholesterol-lowering efficacy of plant sterols/stanols provided in capsule and tablet formats: results of a systematic review and meta-analysis. J Acad Nutr Diet Nov 2013; 113:1494-503

31. Chen JT, Wesley R, Shamburek RD, et al. Meta-analysis of natural therapies for hyperlipidemia:plant sterols and stanols versus policosanol. Pharmacotherapy 2005;25: 171.

32. Berthold HK, Unverdorben S, Degenhardt R, et al. Effect of policosanol on lipid levels among patients with hypercholesterolemia or combined hyerlipidemia.a randomized controlled trial. JAMA 2006;295:2262.

33. Heber D, Yip I, Ashley JM, et al. Cholesterol-lowering effects of a proprietrary Chinese red-yeast-rice dietrary supplement. Am J Clin Nutr 1999;69:231.

34. Wang J, Lu Z, Chi J, et al. Multicenter clinical trial of serum lipid lowering effects of Monascus purpreus (red yeast) rice preparation from traditional Chinese medicine. Cur Ther Res 1997;58:964.

35. Becker DJ, Gordon RY, Halbert SC, et al. Red yeast rice for dyslipidemia in statin-intolerant patients: a randomized trial. Ann Inter Med 2009;150:830.

36. Bunnoy A, Saenphet K, Lumyong S, et al. Monascus purpureus-fermented Thai glutinous rice reduces blood anh hepatic cholesterol and hepatic steatosis concentrations in diet-induced hyercholestrolemic rats. BMC Complement Altern Med 2015;15:88.

37. Patrick L, Uzick M. Cardiovascuar disease: C-reactive protein and inflammatory disease paradigm: HMG-Coa reductase inhibitors, alpha-tocopherol, red yeast rice, and olive oil polyphenols. A review of literature. Altern Med Rev 2001;6:248.

38. Mink PJ, Scrafford CG, Barraj LM, et al. Flavonoid intake and cardiovascular disease mortality: a prospective study in postmenopausal women. Am J Clin Nutr 2007;85:895

39. Chen Q, Wang E, Ma L, et al. Dietary resveratrol increases the expression of hepatic 7a-hydroxylase and ameliorates hypercholesterolemia in high-fat fed C57BL/6J mice. Lipids Health Dis 2012;11:56. 
40. Sahebkar A. Effects of resveratrol supplementation on plasma lipids: A systematic review and meta-analysis of randomized controlled trials. Nutr Rev 2013;71:822-35.

41. $\mathrm{Wu} \mathrm{CH}$, Chend SC, Oud TT, et al. Mulberry leaf polyphenol extracts reduced hepatic lipid accumulation involving regulation of adenosine monophosphate activated protein kinase and lipogenic enzymes. J Funct Foods 2013;5:1620-32.

42. Jin D, Xua Y, Meia X, et al. Antiobesity and lipid lowering effects of thea flavins on high-fat diet induced obese rats. J Funct Foods 2013;5:1142-50.

43. Sabatè J, Haddad E, Tnzman JS, et al. Serum lipid response to graduated enrichment of a Step I diet with almonds: a randomized feedinf trial. Am J Clin Nutr 2003;77:1379.

44. Agostoni C, Bresson J-L, Fairweather-Tait S, et al. EFSA panel on dietetic products, nurition and allergies (NDA). EFSA J 2011;9(4):2033.

45. Castaner O, Covas M, Khymenets O, et al. Protection of LDL from oxidation by olive oil polyphenols is associated with a downregulation of CD40-ligand expression and its downstream products in vivo in humans. Am J Clin Nutr 2012;95:1238-44.

46. Estruch R, Ros E, Salas-Salvadò J, et al. Primary pre- vention of cardiovascular disease with Mediterranean diet. N Engl J Med 2013;368:1279.

47. Fraser GE, Sabatè J, Beeson WL, Strahan TM. A possible protective effect of nut consumption on risk of coronary heart disease. The Adventis Health Study. Arch Intern Med 1992;152:1416.

48. Szapary PO, Wolfe ML, Bloedon LT, et al. Guggulipid for the treatment of hypercholesterolemia: a randomized controlled trial. JAMA 2003;290:765.

49. Rayman MP, Stranges S, Griffin BA, et al. Effect of supllementation with high-selenium yeast on plasma lipid: a randomized trial. Ann Inter Med 2011;154:656.

50. Garden CD, Lawson LD, Block E, et al. Effect of raw garlic vs commercial garlic supplements on plasma lipids concentrations in adults with moderate hypercholesterolemia: a randomized trial. Arch Intern Med 2007;167:346.

51. Ruscica M, Gomaraschi M, Mombelli G, et al. Nutraceutical approach to moderate cardiometabolic risk: results of a randomized, double-blind and crossover study with Armolipid plus. J Clin Lip 2014;8:61-9.

52. Yuwen T, Yike J, Chi-Tang H, et al. Common delivery system for enhancing in vivo bioavailibility and biological efficacy of nutraceuticals. J Funct Foods 2014;7: 112-28. 


\title{
Nutrizione, omega-3 e cancro
}

\author{
Alberto Mazza, Giovanni Rubello, Giulia Mazza \\ UOC di Medicina Interna, Azienda ULSS 18, Ospedale di Rovigo, Italia
}

\section{RIASSUNTO}

È ben assodato che una dieta con alimenti ad alto indice glicemico, di proteine animali e di acidi grassi saturi, favorisce lo sviluppo del cancro. Al contrario, un'alimentazione ricca di frutta, verdure, legumi e l'uso d'olio extravergine d'oliva e di pesce ad alto contenuto in acidi grassi polinsaturi (PUFA), che nel loro insieme costituiscono la base della nota dieta mediterranea, sembra svolgere azioni preventive sul cancro. In particolare i PUFA della serie omega-3, oltre al loro noto ruolo ipo-lipemizzante e di riduzione dell'incidenza degli eventi coronarici fatali, da alcuni anni sono alla ribalta della ricerca scientifica internazionale per la loro capacità di modulare i complessi meccanismi bio-molecolari del cancro, evidenziati in numerosi studi clinici ed epidemiologici. Tuttavia, i dati della letteratura scientifica hanno offerto risultati alquanto contrastanti, tanto che gli omega-3 hanno dimostrato avere un'associazione talora diretta, inversa o addirittura nulla sul rischio di cancro. Tali differenze sembrano derivare da alcuni bias metodologici e procedurali nei diversi studi dove è stata testata la loro efficacia, dalla diversa dose utilizzata e dall'ampia variabilità individuale circa la bio-disponibiltà raggiunta durante il trattamento con omega-3. Oggigiorno, quest'ultima dovrebbe in parte essere migliorata grazie all'introduzione in commercio di prodotti contenenti omega-3 di alta qualità per i quali l'industria farmaceutica, in particolare italiana, ha notevolmente investito per offrire al consumatore prodotti efficaci e sicuri. Nella pratica clinica è ormai ampiamente condivisa l'opinione che gli omega-3 sono efficaci nell'influenzare la carcinogenesi, aprendo nuovi scenari nell'ambito della ricerca oncologica dell'uomo. Non vi sono tuttavia al momento evidenze scientifiche definite sull'efficacia degli omega-3 nella prevenzione primaria del cancro, mentre non vi sono dubbi sulle loro capacità di migliorare la prognosi di alcune neoplasie e di essere ottimi coadiuvanti della terapia anti-neoplastica tradizionale. Infine dovrà essere definita - come per la malattie cardiovascolari sia in prevenzione primaria che secondaria - la dose minima efficace ed $\mathrm{i}$ targets terapeutici da raggiungere dal trattamento con omega-3 nelle diverse forme di cancro. Tuttavia, per rispondere a quest'ultimo quesito dovremo attendere i risultati di alcuni importanti trials di intervento i cui dati saranno disponibili a breve.

\section{Introduzione}

Il progressivo invecchiamento della popolazione generale osservato negli ultimi decenni si è accompagnato ad un aumento dell'incidenza del cancro e recenti studi dimostrano come il $60 \%$ delle neoplasie è attribuibile a fattori alimentari ed abitudini voluttuarie modificabili ${ }^{1}$ e solo nel $15-20 \%$ a fattori primariamente genetici. ${ }^{2} \mathrm{Ne}$ consegue che le cause più frequenti del cancro sono riconducibili a fattori ambientali, comportamentali ed a mutazioni genetiche conseguenti a danni del DNA, in parte controllabili e suscettibili di interventi mirati che nel tempo possono portare a ridurre l'incidenza delle neoplasie. ${ }^{3}$

Infatti, fin dalla seconda metà del secolo scorso,

Corrispondente: Alberto Mazza, UOC di Medicina Interna, Azienda ULSS 18, Ospedale di Rovigo, viale Tre Martiri 140, 45100 Rovigo, Italia.

E-mail: mazza.alberto@azisanrovigo.it

Articolo pubblicato secondo la Creative Commons Attribution NonCommercial 3.0 License (CC BY-NC 3.0).

(C) Copyright A. Mazza et al., 2015

Licensee PAGEPress, Italy

QUADERNI - Italian Journal of Medicine 2015; 3:288-300 numerosi studi scientifici avevano rilevato l'esistenza di una stretta relazione tra alimentazione e rischio di cancro. ${ }^{4-8}$ In particolare, la componente qualitativa dei cibi ha dimostrato svolgere un ruolo preminente nel favorire o al contrario prevenire l'insorgenza delle neoplasie $^{9}$ e grazie ad importanti studi di biologia molecolare è stato in seguito possibile comprendere ciò che è stato tramandato dalla tradizione popolare millenaria circa il ruolo dell'alimentazione sul benessere dell'uomo. ${ }^{10}$

Per quanto concerne il rapporto tra alimentazione e cancro è ormai ben noto che un regime dietetico costituito da alimenti ad alto indice glicemico, un eccesso di proteine animali ed in particolar modo un elevato consumo di carne rossa ${ }^{11}$ e di acidi grassi saturi, ${ }^{12}$ come anche inadeguate abitudini voluttuarie che spesso si accompagnano ad un scorretta alimentazione - quali tabagismo ed elevato consumo di alcool ${ }^{13}$ - favoriscano lo sviluppo del cancro. Al contrario, un'alimentazione ricca di frutta, verdure, legumi, l'uso quotidiano d'olio extravergine d'oliva e di pesce ad alto contenuto in acidi grassi polinsaturi (PUFA) che nel loro insieme costituiscono la base della nota dieta mediterranea, sembrano svolgere azioni preventive sul cancro. ${ }^{14,15}$

In particolare i PUFA della serie omega-3 oltre alla loro più nota capacità di ridurre le concentrazioni 
plasmatiche dei trigliceridi, ${ }^{16}$ noti fattori di rischio per malattie cardiovascolari, sembrano influenzare l'aggregazione piastrinica e la modulazione di alcuni meccanismi dell'infiammazione ${ }^{17}$ e del sistema immunitario responsabili dell'insorgenza del cancro. ${ }^{18}$ Al contrario i PUFA della serie omega-6 sembrano svolgere attraverso l'attivazione di complessi sistemi enzimatici e bio-molecolari un'azione esattamente opposta, favorendo lo sviluppo del cancro. I PUFA sono acidi grassi cosiddetti essenziali in quanto contribuiscono alla formazione della struttura della membrana cellulare, hanno un ruolo chiave nelle vie dei segnali intra-cellulari e nella funzione delle cellule epiteliali, ${ }^{19}$ ma essendo sintetizzati solo in piccola parte dall'organismo devono essere assunti principalmente con la dieta. ${ }^{20}$ Tuttavia, al di là dei loro effetti benefici evidenziati in numerosi ambiti della medicina, in particolare a livello cardiovascolare, nel corso degli anni sono comparsi nella letteratura scientifica oncologica alcuni studi con risultati contrastanti dove è stato addirittura osservato un maggior rischio di alcune forme di cancro nei soggetti che assumevano elevate quantità di PUFA tipo omega-3. ${ }^{21}$ Ciò sembra in parte dovuta all'ampia variabilità individuale della bio-disponibiltà ${ }^{22}$ e che potrebbe in parte spiegare il loro diverso ruolo osservato in letteratura a seconda o meno del raggiungimento di una soglia ematica potenzialmente efficace sul cancro. Tale ampia variabilità della bio-disponibilità è ascrivibile non solo alla dose giornaliera assunta, ma anche da fattori ambien- tali, genetici ${ }^{23,24}$ e dalla qualità dei diversi prodotti contenenti omega-3. Infine, diversamente dalle malattie cardiovascolari (sia in prevenzione primaria che secondaria) e per la tutela della salute nella popolazione generale, dove l'Organizzazione Mondiale della Sanità e le principali società scientifiche del mondo hanno da tempo stabilito le dosi efficaci ed i targets terapeutici da raggiungere dal trattamento con omega3 (Tabella 1), per il cancro tali raccomandazioni sono ancora lontane da essere definite.

Scopo di questa monografia è di analizzare i meccanismi fisiopatologici e molecolari alla base delle complesse relazioni tra nutrizione e cancro, discutendo in particolare il possibile ruolo degli omega-3 nella prevenzione e nel trattamento delle neoplasie alla luce delle più recenti evidenze scientifiche.

\section{Messaggio chiave}

Il $60 \%$ delle neoplasie è attribuibile a fattori alimentari, in particolare con l'assunzione d'elevate quantità d'acidi grassi saturi. Al contrario, un'alimentazione ricca di PUFA presenti principalmente nel pesce e che costituiscono la base della dieta mediterranea, sembra svolgere un'azione preventiva sul cancro. In realtà tale ruolo non è ancor ben definito, tanto che gli omega-3 assunti con la dieta hanno dimostrato avere nella letteratura scientifica un'associazione inversa, diretta o addirittura nulla sul rischio di cancro.

Tabella 1. Quantità di acidi grassi polinsaturi omega-3 da introdurre con la dieta, raccomandate per la popolazione generale e per specifiche categorie di soggetti, da parte di società scientifiche e da autorità nazionali nei principali stati del mondo.

\begin{tabular}{|c|c|c|c|}
\hline Paese & Organizzazione/Società & Target dei soggetti & Quantità \\
\hline \multirow[t]{2}{*}{ Mondo } & World Health Organization (WHO) & Popolazione generale & PUFA: $1-2 \%$ delle calorie giornaliere \\
\hline & $\begin{array}{l}\text { International Society for the Study of Fatty Acids } \\
\text { and Lipids (ISSFAL) }\end{array}$ & Popolazione generale & Almeno 500 mg EPA+DHA/die \\
\hline \multirow[t]{2}{*}{ USA } & American Heart Association & Popolazione generale & Pesce con $\geq 500 \mathrm{mg}$ di EPA+DHA \\
\hline & Academy of Nutrition and Dietetics & Popolazione generale & $500 \mathrm{mg}$ EPA+DHA/die \\
\hline \multirow[t]{2}{*}{ Europa } & $\begin{array}{l}\text { Expert Workshop of the European Academy } \\
\text { of Nutritional Sciences }\end{array}$ & Popolazione generale & $\begin{array}{l}\text { Se non uso di pesce, integrazione } 200 \mathrm{mg} \\
\text { EPA+DHA/die }\end{array}$ \\
\hline & $\begin{array}{l}\text { Fifth Joint Task Force of the European Society } \\
\text { of Cardiology e Other Societies on Cardiovascular } \\
\text { Disease Prevention in Clinical Practice }\end{array}$ & $\begin{array}{l}\text { Popolazione generale } \\
\text { per ridurre rischio } \\
\text { cardiovascolare }\end{array}$ & Pesce almeno 2 volte/sett, di cui una, pesce azzurro \\
\hline Italia & Ministero della Salute & $\begin{array}{l}\text { Donne in gravidanza } \\
\text { e allattamento }\end{array}$ & $\begin{array}{l}\text { Per le donne vegane consumare alimenti ricchi } \\
\text { di DHA }\end{array}$ \\
\hline Australia & National Heart Foundation & Popolazione generale & $\begin{array}{l}500 \mathrm{mg} \text { EPA }+ \text { DHA/die, ottenuti da pesce, capsule } \\
\text { di olio di pesce, o cibi e bevande arricchite }\end{array}$ \\
\hline Canada & Ministero della Salute & Popolazione generale & 1,2-1,6 g/die di PUFA \\
\hline Giappone & Ministero della Salute & Popolazione generale & $>1 \mathrm{~g} \mathrm{EPA}+\mathrm{DHA} / \mathrm{die}$ \\
\hline
\end{tabular}

PUFA, acidi grassi polinsaturi omega-3; EPA, acido eicosapentaenoico; DHA, acido docosaesaenoico. 


\section{Biochimica e metabolismo degli acidi grassi polinsaturi}

Gli acidi grassi polinsaturi, sulla base della posizione del loro primo doppio legame nella porzione metilica sono principalmente suddivisi in 2 classi: ${ }^{25}$ gli omega-3 e gli omega-6 (Figura 1). I PUFA omega3 più comuni sono l'acido $\alpha$-linolenico, l'acido eicosapentaenoico o EPA e l'acido docosaesaenoico o DHA e sono definiti tali perché hanno il doppio legame in posizione 3. Questi ultimi, sono i più importanti PUFA poichè svolgono nell'organismo umano importanti attività strutturali e funzionali. In particolare il DHA ha un ruolo importante nello sviluppo e nella maturazione cerebrale, dell'apparato riproduttivo e del tessuto retinico esercitando una funzione strutturale nei fosfolipidi delle sinapsi cerebrali, nella retina e nei canali intra-membrana del sodio e del calcio in particolare a livello dei miocardiociti. ${ }^{26}$ L'EPA è invece il principale precursore delle prostaglandine della serie 3, che come noto possiedono un'importante attività antiaggregante piastrinica. ${ }^{27}$

D'altra parte, i più importanti PUFA della serie omega-6 sono l'acido linoleico (LA) e l'acido ara- chidonico (AA). Al pari dei PUFA omega-3 anche gli omega-6 svolgono un ruolo strutturale e funzionale: l'AA è presente nei fosfolipidi di membrana ed è importante, opportunamente bilanciato con il DHA, nello sviluppo embrionale; produce inoltre le prostaglandine della serie 2 dando luogo alla formazione di intermedi metabolici ad attività pro-infiammatoria e aggregante piastrinica come il trombossano-A2. ${ }^{28}$ L'organismo umano è capace di produrre tutti i PUFA necessari, eccetto due: l'acido $\alpha$-linolenico (ALA, omega-3) e l'acido linoleico (LA, omega-6). Tuttavia nell'uomo ALA e LA sono metabolizzati dagli stessi enzimi (Figura 1) e questo meccanismo è da tenere in considerazione in quanto un elevato rapporto omega-6/omega-3 (come di seguito descritto) da eccessiva assunzione di LA con la dieta, potrebbe ridurre la quantità di suddetti enzimi disponibili per il metabolismo di ALA e con conseguente aumento del rischio di alcune malattie, tra cui il cancro. ${ }^{29}$

\section{Messaggio chiave}

I PUFA omega-3 e omega-6 sono definiti essenziali per le loro numerose e fondamentali funzioni molecolari e biochimiche nell'organismo; devono essere

\section{Omega-3}

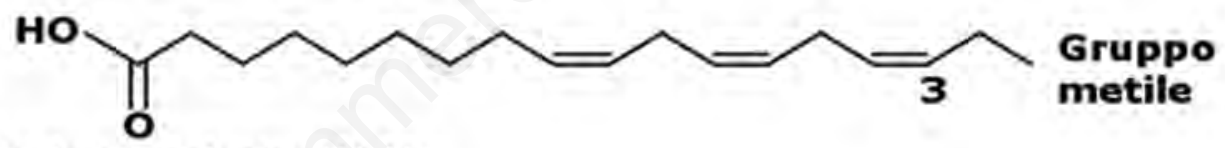

Acido a-Linolenico (ALA)<smiles>CCC=CCC=CCC=CCC=CCC=CCCCC(=O)O</smiles>

Acido Eicosapentaenoico (EPA)<smiles>CC/C=C/C/C=C/C/C=C/C/C=C/C/C=C/C/C=C/CCC(=O)O</smiles>

Acido Docosaesaenoico (DHA)

\section{Omega-6}<smiles>CCCCCC=CCC=CCCCCCCCC(=O)O</smiles><smiles>CCCCCC=CCC=CCC=CCC=CCCCC(=O)OC(C)(C)C(C)=O</smiles>

Figura 1. Struttura molecolare degli acidi grassi polinsaturi tipo omega-3 ed omega-6. 
tuttavia assunti in uguale quantità, in quanto un aumento del rapporto omega-6/omega-3 sembra essere un potenziale fattore di rischio per il cancro.

\section{Carcinogesi e acidi grassi polinsaturi}

Numerosi studi sostengono l'ipotesi che i PUFA omega-3 possono inibire la formazione e la progressione del cancro ${ }^{30}$ ed addirittura svolgere un possibile ruolo terapeutico per alcune neoplasie. ${ }^{31}$ Tuttavia, anche se studi sperimentali su modelli animali hanno dimostrato che gli omega-3 influenzano il microambiente della carcinogenesi inibendo la cascata infiammatoria ad essa associata, favorendo l'apoptosi ed esercitando effetti anti-proliferativi ed inibenti la produzione di alcuni dei principali mediatori angiogenici, ${ }^{32}$ il reale meccanismo d'azione dei PUFA omega-3 nella modulazione dei meccanismi bio-molecolari favorenti il cancro non è ancora ben definito. D'altra parte, a rendere ancora più complesso il ruolo dei PUFA sulla carcinogesi, alcuni studi sempre su modelli animali, hanno dimostrato che gli omega-6, diversamente dagli omega3 , sembrano avere un ruolo favorente l'insorgenza del cancro (Figura 2). In particolare alcuni Autori hanno evidenziato che una dieta ricca di omega-6 induce nell'uomo un aumento dello stress ossidativo con perossidazione dei fosfolipidi di membrana e formazione di radicali liberi intra-cellulari che a loro volta inducono alterazioni del DNA ed innescano la carcinogenesi. ${ }^{33}$ Alla luce di queste evidenze, negli ultimi anni è cresciuto l'interesse della ricerca sul rapporto tra omega-6 e omega- 3 e rischio di cancro, ${ }^{34}$ tanto che alcuni Autori affermano che la relazione tra PUFA e cancro possa dipendere da una questione di bilancio tra questi due acidi grassi. In particolare, tanto più elevato è il rapporto omega-6/omega-3, tanto maggiore è il rischio di cancro. Infatti è noto già da tempo che in modelli animali in cui veniva artificialmente indotta la crescita di un tumore, una dieta ad elevato contenuto di PUFA omega-6 aumentava in modo significativo la mortalità nei ratti trattati rispetto ai controlli. ${ }^{35}$ Viceversa, negli stessi animali la sopravvivenza aumentava in modo significativo, se veniva loro somministrata una dieta ad alto contenuto di omega- $3^{36}$ e negli stessi animali era possibile rilevare anche una riduzione delle dimensioni della massa tumorale. Tali conoscenze sono state poi trasferite anche nell'uomo dove è stata evidenziata una possibile relazione tra un alterato rapporto omega-6/omega-3 e rischio di cancro mammario ${ }^{37,38}$ e del colon. ${ }^{39,40}$ Tuttavia,

\section{Omega-3}

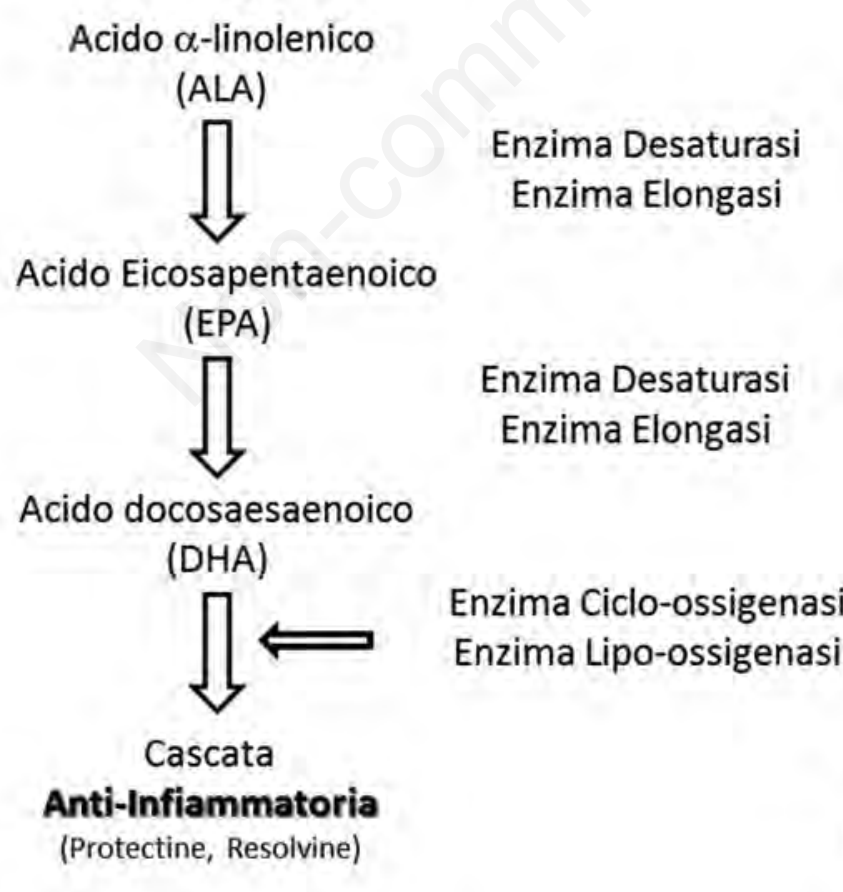

Anti-Cancerogena?

\section{Omega-6}

\author{
Acido $\alpha$-linoleico
}

(LA)

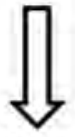

Acido Arachidonico

(AA)

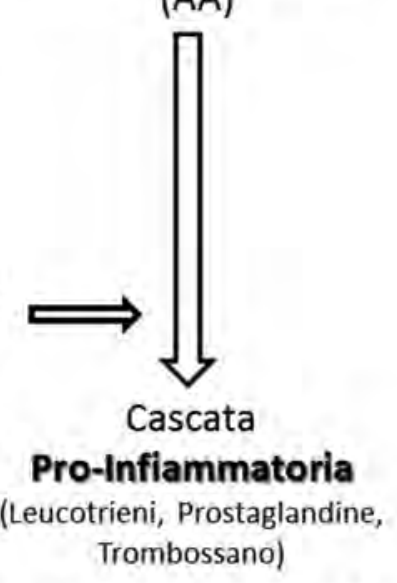

Pro-Cancerogena?

Figura 2. Vie metaboliche di attivazione degli acidi grassi poli-insaturi omega-3 ed omega-6 e loro prodotti terminali con possibile attività pro- ed anti-cancerogena. 
contrariamente ai risultati sperimentali in modelli animali, nelle neoplasie dell'uomo il ruolo di tale rapporto non è ancora ben definito, tanto che molti esperti suggeriscono che il maggior rischio di cancro non è tanto legato ad un aumento del rapporto omega-6/omega-3, ma soprattutto alla quantità assoluta di acidi grassi assunti con la dieta.

\section{Messaggio chiave}

I PUFA omega-3 influenzano il microambiente della carcinogenesi, modulando la cascata infiammatoria, favorendo l'apoptosi ed esercitando effetti antiproliferativi ed inibenti la produzione di alcuni dei principali mediatori angiogenici. ${ }^{32}$ Al contrario $i$ PUFA omega-6, sembrano avere un ruolo favorente l'insorgenza del cancro.

\section{Omega-3 e prevenzione del cancro}

Nel mondo scientifico il ruolo degli omega-3 come molecole anti-cancro è ancora ampiamente dibattuto, in particolare a seguito di risultati contrastanti emersi nei pochi trials, per lo più derivati come frangia marginale di studi condotti primariamente per verificare il ruolo degli omega-3 nella prevenzione delle malattie cardiovascolari. ${ }^{41}$ In particolare un'analisi post-hoc di un trial italiano randomizzato includente 2501 soggetti con malattia cardiovascolare nota, ha evidenziato solo nel sottogruppo di sole femmine un'associazione diretta tra la supplementazione con $600 \mathrm{mg}$ di EPA e DHA e aumentato rischio di cancro. ${ }^{42}$ Questi risultati devono essere interpretati con cautela, in quanto derivanti da un sottogruppo, dove l'incidenza di casi di cancro era di 29 casi.

In realtà la maggior parte delle conoscenze oggi disponibili circa la relazione tra omega-3 e cancro derivano da studi che hanno considerato maggiormente l'efficacia della dieta ricca di olio pesce - principale fonte di omega-3 - sull'insorgenza di neoplasie. Infatti, contrariamente a quanto sopra, in più ampi studi randomizzati l'assunzione di olio di pesce non ha evidenziato alcuna relazione con l'aumento del rischio di cancro $^{43}$ ed addirittura in modelli animali i benefici dell'olio di pesce nelle riduzione dell'incidenza e della prognosi di molte neoplasie è stato ampiamente dimostrato. ${ }^{44,45}$ In particolare i dati provenienti da revisioni sistematiche e da meta-analisi di ampi studi osservazionali e prospettici hanno evidenziato effetti protettivi del consumo di pesce sul rischio di qualsiasi tipo di cancro. ${ }^{46-48}$

Risultati contrastanti sono invece emersi tra uso di omega-3 e cancro della prostata dove alcuni studi ${ }^{49,50}$ avevano inizialmente suggerito una associazione tra dieta con elevati livelli di omega-3 e rischio di tale cancro; tuttavia in una meta-analisi di questi studi non è emersa alcuna associazione statisticamente significativa tra i livelli di omega-3 e rischio di tale neoplasia. ${ }^{51,52}$ Sono qui di seguito descritte le principali evidenze oggi disponibili in letteratura sull'associazione tra l'uso degli omega-3 e le più frequenti forme di cancro nel maschio e nella femmina.

\section{Messaggio chiave}

Non vi sono evidenze in letteratura sull'efficacia degli omega-3 nella prevenzione primaria del cancro e per nessun dosaggio, tanto che ne viene sconsigliato l'uso in particolare a dosi elevate.

\section{Omega-3 e cancro della prostata}

Il carcinoma della prostata rappresenta la neoplasia più comune del sesso maschile nel mondo occidentale ed è al secondo posto come causa di morte tra tutte le neoplasie con un'incidenza in progressivo aumento, ${ }^{53}$ in gran parte dovuta al progressivo invecchiamento della popolazione generale. ${ }^{54}$. Numerosi studi hanno cercato di definire le fasi della cancerogenesi del tumore prostatico evidenziando come oltre alla nota stimolazione ormonale sul recettore androgenico, ${ }^{53}$ una dieta ricca di acidi grassi omega- 6 promuove la crescita dello stesso. ${ }^{55}$ In particolare, un eccesso di acido $\alpha$-linoleico, tipico delle diete occidentali, determina la stimolazione di agenti infiammatori quali ciclossigenasi, leucotrieni e prostaglandine che come sopra descritto (Figura 2) hanno un possibile ruolo nella carcinogenesi. Queste ipotesi trovano conferma in studi epidemiologici dove 1'elevato consumo di omega-6 nella dieta osservato negli ultimi 50 anni nei paesi occidentali ha determinato un incremento del rapporto omega-6/omega-3 sopra descritto, dove tale squilibrio potrebbe rappresentare un fattore di attivazione di carcinomi prostatici presenti allo stato latente. ${ }^{56} \mathrm{Al}$ contrario, altri Autori hanno evidenziato un ruolo protettivo sullo sviluppo e la progressione del cancro della prostata con una dieta ricca di acidi grassi omega-3, in particolare grazie all'attività anti-antinfiammatoria ed inibente la carcinogenesi dell'EPA e del DHA (Figura 2). In particolare è stato sottolineato che l'azione anti-infiammatoria e di inibizione della carcinogenesi prostatica degli omega-3 avviene attraverso il blocco ${ }^{57}$ della via enzimatica della ciclo-ossigenasi tipo-2. In realtà in una recente metanalisi è stato evidenziato che non tutti gli omega-3 svolgono un ruolo protettivo in quanto un incremento del consumo di ALA di derivazione animale può associarsi al rischio di carcinoma prostatico. ${ }^{58}$ Questo apparente effetto negativo degli omega-3 sembra essere collegato alla denaturazione dell'ALA di origine animale quando portato a temperature elevate dove avviene un processo di perossidazione lipidica e di formazione di radicali liberi, noti fattori pro-cancerogeni. ${ }^{59}$ Tuttavia in un re- 
cente studio condotto presso la Washington State University è stato evidenziato che gli omega-3 si legano a loro specifici recettori delle cellule tumorali, denominati free fatty acid receptor 4 , inibendo il processo di proliferazione del cancro prostatico. Questa ricerca è la prima a dimostrare l'efficacia degli acidi grassi omega3 nel campo dei tumori, in quanto è stata effettuata su cellule cancerogene di prostata umana ${ }^{60} \mathrm{La}$ conferma di un ruolo protettivo degli omega-3 sul cancro prostatico deriva da una recentissima metanalisi di Alexander et $a l .{ }^{61}$ che ha incluso studi nei quali il ruolo degli omega- 3 è stato analizzato non tanto come quantità di omega-3 assunti con la dieta ma con l'uso di biomarcatori ematici e cellulari degli stessi. Tali risultati negano quelli di altre metanalisi comparse nel 2013, che oltre a non evidenziare alcun ruolo degli omega-3 sulla carcinogenesi prostatica, avevano addirittura ipotizzato una loro diretta associazione con un maggior il rischio di tale neoplasia. ${ }^{21,49}$

\section{Messaggio chiave}

I PUFA omega-3, NON sono fattori di rischio per il cancro della prostata, ma al contrario svolgono un ruolo protettivo legandosi a loro specifici recettori denominati free fatty acid receptor 4 , che inibiscono il processo di proliferazione del cancro prostatico.

\section{Omega-3 e cancro della mammella}

Il cancro del seno è uno dei tumori più frequenti della popolazione generale femminile ed è responsabile di circa il $25 \%$ dei casi di tutte le morti per cancro ${ }^{62}$ Negli ultimi decenni, numerosi studi epidemiologici ${ }^{63,64}$ hanno evidenziato che una dieta sana ed un adeguato stile di vita sono fondamentali per la prevenzione di tale neoplasia, mentre una dieta ricca di acidi grassi ne aumenta fortemente il rischio. ${ }^{65-69} \mathrm{In}$ particolare tra i diversi PUFA assunti con la dieta, quelli tipo omega-3 si sono dimostrati capaci di inibire o di modulare la carcinogenesi del cancro del seno, sia in studi condotti su modelli animali ${ }^{70,71}$ che in vitro su colture cellulari. ${ }^{72} \mathrm{Al}$ contrario nell'uomo i risultati di studi osservazionali e caso-controllo sono alquanto contrastanti, in quanto gli omega-3 assunti con la dieta hanno dimostrato avere un'associazione talora inversa, ${ }^{66,73}$ diretta $^{74,75} \mathrm{o}$ addirittura nulla ${ }^{66,76-79}$ sul rischio di tale cancro. In una metanalisi condotta da ricercatori Cinesi $^{80}$ su un'ampia popolazione di oltre 880.000 soggetti con oltre 20.000 casi di tumore al seno riscontrati negli Stati Uniti, Europa ed Asia, sarebbero necessarie una o due assunzioni settimanali di pesce ricco di PUFA, per ottenere una riduzione significativa nell'incidenza delle neoplasie mammarie. In altre parole, questi risultati sembrano sostenere le proprietà benefiche degli omega-3, dove le donne con una dieta ricca di pesce hanno un rischio del 14\% inferiore di contrarre il tumore, rispetto a quelle che ne consumano poco o addirittura nulla. È tuttavia doveroso sottolineare, che tale positiva associazione tra riduzione del rischio di cancro mammario ed omega-3 non è casuale, e sembra in gran parte correlata al consumo di pesce tipico delle popolazioni asiatiche, dove il consumo di pesce è culturalmente più elevato. Tuttavia tali possibili benefici non possono essere ricondotti al consumo occasionale: la diminuzione del rischio è infatti molto più forte nelle donne in menopausa, come a dire che gli effetti benefici aumentano dopo molti anni. I ricercatori hanno inoltre notato una correlazione positiva già con una blanda assunzione di omega-3: ne basterebbero $0,1 \mathrm{~g}$ al giorno (un piatto di pesce alla settimana circa) per ottenere una riduzione del rischio del $5 \%$. La ricerca invece esclude, o perlomeno non conferma, alcun effetto benefico sul rischio di cancro al seno del consumo di omega-3 vegetali, come ad esempio quelli contenuti nelle noci e negli oli di lino, di colza e di ribes nero. Tali risultati negano l'esperienza di alcuni ricercatori dell'Università di Toronto, che hanno studiato gli effetti dell' assunzione di semi di lino da parte dei ratti. ${ }^{81}$ In particolare è stata osservato che questi semi, oltre ad essere ricchi di omega-3 (acido $\alpha$-linolenico) contengono anche elevate quantità di lignano, una molecola che è in grado di bloccare la produzione degli estrogeni. Entrambe queste molecole, spiegano gli Autori, possono ridurre la dimensione dei tumori al seno del $50 \%$, ma mentre l'omega-3 è più efficace in caso di forme di cancro già diagnosticate, il lignano esercita la sua azione antitumorale bloccando la formazione di nuove neoplasie. Uno studio Americano ha invece dimostrato in modelli animali che l'assunzione di omega-3 in ratti gravidi con livelli significativamente elevati di estrogeni riduce la probabilità che i loro nati sviluppino un cancro al seno. ${ }^{82}$ Infine, uno studio francese ha dimostrato che in caso di tumore alla mammella i livelli di DHA presente nei tessuti adiposi è un fattore in grado di predire la sensibilità alla chemioterapia. ${ }^{83}$

\section{Messaggio chiave}

I PUFA omega-3, ma solo quelli di origine animale in particolare se contenuti nell'olio di pesce, inibiscono la proliferazione del cancro mammario sia in modelli animali che nell'uomo; per quest'ultimo si sono dimostrati ottimi coadiuvanti per la chemioterapia.

\section{Omega-3 e cancro del colon}

Il cancro del colon rappresenta la terza neoplasia più frequente della popolazione generale ${ }^{84} \mathrm{ed} \mathrm{è} \mathrm{quindi}$ importante individuare i potenziali agenti preventivi per ridurne l'incidenza. ${ }^{85,86}$ Come sopra accennato, 
l'infiammazione è riconosciuta essere uno dei principali meccanismi d'innesco della cancerogenesi e come dimostrato in alcuni trial clinici d'intervento i PUFA omega-3 si sono dimostrati efficaci nel ridurre l'infiammazione nell'uomo. ${ }^{87,88}$ Tali evidenze derivano da studi epidemiologici condotti alla fine del secolo scorso, dove alcuni ricercatori sudafricani dimostrarono che una dieta ricca di pesce, principale fonte di omega-3, riduceva l'insorgenza del cancro del colon. ${ }^{89}$ In particolare confrontando l'incidenza del cancro tra gli abitanti di Città del Capo e i pescatori residenti in villaggi situati sulla costa sud-africana, coloro che abitavano in città - nonostante seguissero una dieta salutare con elevate quantità di frutta e verdura, alimenti con note proprietà protettive sul cancro al colon - avevano una probabilità di sviluppare il cancro del colon 6 volte maggiore che negli abitanti dei villaggi costieri. Le analisi ematiche dei soggetti arruolati nello studio svelarono che chi viveva in riva al mare aveva livelli di omega-3 circa 3 volte superiori rispetto agli abitanti che vivevano in città. Negli anni successivi ricercatori italiani hanno dimostrato che il trattamento giornaliero con dose elevate di omega-3, in particolare con 4,1 g di EPA e 3,6 g di DHA, in pazienti con polipi del colon pre-cancerosi era in grado di diminuire in sole 2 settimane la proliferazione cellulare. ${ }^{90}$ Inoltre, tale trattamento è risultato ben tollerato e privo di effetti collaterali significativi e tali ricercatori hanno affermato che l'olio di pesce rappresenta un valido aiuto per gli individui ad alto rischio di cancro al colon. Questi risultati sono stati poi confermati anche nella popolazione americana, dove l'assunzione regolare di olio di pesce oltre ad essere ben tollerato, è risultata efficace nel prevenire la comparsa di nuovi polipi precancerosi. ${ }^{91}$ Tali dati sono stati ampiamente confermati da una meta-analisi del 2014, anche se è stato sottolineato che i reali benefici di una dieta con supplementi di EPA e DHA sono diversi tra maschi e femmine, dipendono dalla sede della neoplasia e da caratteristiche genetiche individuali. ${ }^{92}$

\section{Messaggio chiave}

I PUFA omega-3 a dosi elevate, rallentano la trasformazione delle neoformazioni pre-cancerose, in particolare nei soggetti ad aumentato rischio per famigliarità di tale cancro.

\section{Omega-3 e cancro del polmone}

La neoplasia del polmone rappresenta la principale causa di morte per cancro nel mondo, stimata essere di circa 1200 morti all'anno. Tuttavia, nonostante l'ampia ricerca condotta negli ultimi anni mirata in gran parte nella comprensione della biologia molecolare e dei progressi osservatisi nella diagnostica, nella chirurgia e nella chemioterapia, la percentuale di sopravvivenza a 5 anni del cancro del polmone è ancora molto scarsa (15-20\% dei casi). Contrariamente ad altre neoplasie dove il ruolo dei PUFA omega-3 è ormai confermato da numerose ricerche, per il cancro del polmone la letteratura scientifica offre osservazioni più limitate, nelle quali sono state per lo più studiati i meccanismi molecolari modulati dai PUFA omega-3 sulla carcinogenesi di tale neoplasia. Tra questi, un ruolo ampiamente trattato riguarda il ruolo svolto dagli omega-3 sulla modulazione di alcuni tipi di recettori nucleari noti come peroxisome proliferator activated receptors $\gamma$ (PPARs$\gamma$ ), la cui attivazione da parte degli omega-3 determina una riduzione della risposta infiammatoria che costituisce una delle fasi della carcinogenesi intracellulare. ${ }^{93}$ In particolare un gruppo di ricercatori Italiani nel 2007 ha addirittura evidenziato un effetto anti-proliferativo dei PUFA omega-3 sella crescita di cellule di adenocarcinoma polmonare mediata dall'attivazione attivazione dei PPARs- $\gamma$ tramite una loro attività di modifica della struttura lipidica della membrana cellulare che la rende meno suscettibile all'aggressione di carcinogeni. ${ }^{94}$ In seguito l'attività antitumorale dei PPARs- $\gamma$ è stata collegata all'attivazione delle integrine, proteine eterodimeriche trans-membrana che non solo svolgono un ruolo centrale nel mediare l'adesione delle cellule alla matrice extracellulare, ma che si comportano come veri e propri recettori, capaci di inviare segnali all'interno della cellula in grado di regolare il differenziamento e la proliferazione. In altre parole l'ipotesi più accreditata è che il danno degli alveoli polmonari da cancerogeni, provochi un aumento transitorio della produzione di integrine ed un conseguente aumento nell'espressione dei relativi recettori a livello cellulare, situazione che potrebbe contribuire a promuovere la proliferazione cellulare ${ }^{95}$ Un ulteriore meccanismo che spiega il possibile ruolo protettivo degli omega-3 nella patogenesi del cancro del polmone deriva dalla loro capacità di inibire l'attività delle prostaglandine della serie 3 che come noto possiedono attività anti-infiammatoria..$^{96} \mathrm{In}$ fine sembra che l'assunzione di PUFA omega-3 sia in grado influenzare favorevolmente la qualità di vita, la performance status e l'attività fisica di pazienti con cancro del polmone in trattamento chemioterapico. Ł̀ tuttavia necessario, come sostengo alcuni esperti, che per la conferma di tali dati si deve individuare e definire l'effetto dose-risposta di PUFA omega-3 da assumere durante il trattamento della neoplasia. ${ }^{97}$

\section{Messaggio chiave}

La relazione tra PUFA omega-3 e cancro del polmone è stata meno studiata in letteratura. È invece ben definita la modulazione sulla carcinogenesi degli omega-3, che avviene attraverso l'attivazione di alcuni recettori nucleari noti come PPARs- $\gamma$. 


\section{Omega-3 come coadiuvanti delle terapie antitumorali}

Le considerazioni sopra descritte derivanti dalla letteratura scientifica devono essere attentamente interpretate, tanto che al momento l'uso degli omega-3 non rappresenta un trattamento ben definito delle neoplasie. Infatti, le esperienze raccolte nel corso degli anni suggeriscono che i PUFA omega-3 possono fornire ottimi risultati quando utilizzati in combinazione con le cure tradizionali del cancro. Un esempio di tale efficacia additiva è nota da oltre 15 anni, quando un gruppo di ricercatori Inglesi testò le potenzialità degli omega-3 nel trattamento di pazienti oncologici affetti da cachessia neoplastica. ${ }^{98}$ Com'è noto, la cachessia è una condizione di dimagrimento estremo associato a deperimento fisico e mentale, che colpisce circa la metà degli individui malati di cancro e che può rappresentare la causa di decesso nel $10-25 \%$ dei pazienti oncologici. In particolare tali ricercatori dimostrarono che la somministrazione di EPA è sufficiente a ridurre significativamente la perdita di peso in soli tre mesi. Un altro esempio significativo dell'effetto coadiuvante degli omega-3 alla tradizionale terapia anti-tumorale è stato enfatizzato in una recente meta-analisi dove il loro utilizzo sembra potenziare l'effetto della radio- e della chemioterapia. ${ }^{99}$ Tale effetto è riconducibile alla loro attività anti-ossidante che probabilmente riduce in parte l'azione dei radicali liberi generati dall'azione dei farmaci chemioterapici che per il loro meccanismo danneggiano le membrane delle cellule neoplastiche. Questi risultati trovano conferma anche in modelli animali nei quali è stato impiantato del tessuto proveniente da un cancro del seno umano, dove il trattamento chemioterapico con tamoxifene combinato all'assunzione degli omega-3, amplificava di circa 10 volte l'efficacia del farmaco e riducendo il tasso di crescita della neoplasia. ${ }^{100}$ Inoltre sembra che la tossicità di alcuni chemioterapici venga molto attenuata dalla contemporanea di assunzione di olio di pesce contente omega-3. ${ }^{101}$

\section{Messaggio chiave}

I PUFA omega-3 possono fornire ottimi risultati nel trattamento del cancro solo quando utilizzati in combinazione con le cure tradizionali e principalmente per la loro attività anti-ossidante che riduce l'azione dei radicali liberi generati dall'azione dei chemioterapici.

\section{Omega-3 nel trattamento delle metastasi}

Un'altra interessante potenzialità degli acidi grassi omega-3 in ambito oncologico, riguarda la loro capacità di modulare il fenomeno della metastasi. ${ }^{102}$ È noto che la cellula tumorale affinché possa migrare lontano dalla massa cancerosa di partenza e colonizzare altre aree dell'organismo è necessario che prima aderisca ai tessuti degli organi bersaglio e che in seguito penetri attraverso la membrane cellulare degli stessi. L'attività anti-metastatica degli omega-3 sembra riconducibile alla loro capacità di inibire l'adesione delle cellule tumorali ai tessuti, principalmente bloccando la produzione dell'enzima collagenasi. Questa molecola, è infatti necessaria per alterare le membrane e permettere alle cellule cancerose di attraversarle. ${ }^{103}$ Tali evidenze trovano ancor una volta conferma in esperimenti condotti in vitro ed in modelli animali circa 20 anni fa quando alcuni ricercatori statunitensi dimostrarono che in ratti nei quali veniva rimosso chirurgicamente un cancro al seno, la dieta ricca degli omega-3 EPA e DHA riduceva la probabilità di diffusione della neoplasia. ${ }^{104}$ Alcuni anni dopo altri ricercatori dell'Università del Texas, dimostrano sempre in modelli animali, che tanto più ricca era l'alimentazione con omega-3 tanto minore era il volume delle eventuali metastasi che si formavano in ratti affetti da tumore mammario. ${ }^{105}$ Tali evidenze in modelli animali hanno trovato recentemente conferma anche in studi in vitro condotti su cellule umane, ${ }^{106}$ aprendo scenari futuri dove donne con cancro del seno potrebbero beneficiare degli stessi effetti arricchendo la loro dieta con EPA e DHA.

\section{Messaggio chiave}

I PUFA omega-3, sembrano modulare la diffusione metastatica del cancro, bloccando la produzione della collagenasi, enzima necessario per alterare le membrane e far penetrare le cellule cancerose nei tessuti bersaglio.

\section{Conclusioni}

La notevole mole di dati presenti nella letteratura scientifica a favore dei benefici degli omega-3 sul cancro, ha parallelamente determinato un progressivo aumento del consumo di tali sostanze a livello della popolazione generale. Come in altri settori della medicina, cresce spontaneo il quesito se l'aumento dell'uso degli omega-3 osservatosi nell'ultimo decennio anche in Europa sia riconducibile più ad interessi economico-commerciali che a reali benefici sulla prevenzione e trattamento del cancro. A tal riguardo uno studio recentemente condotto da una compagnia Svizzera molto quotata in ricerche di mercato nel campo alimentare e nei prodotti nutrizionali afferma che in Europa nel 2015 l'uso degli omega-3 è aumentato del $4 \%$ nel Regno Unito e in Germania e dell' $8 \%$ in Russia. ${ }^{107}$ È stato inoltre evidenziato che nella popolazione generale la dose media di omega-3 assunta è molto va- 
riabile e che vi è opinione comune che l'uso di dosi più elevate di tali sostanze sia più efficace nel prevenire il cancro. Tale convinzione deriva in parte dall'interpretazione di affermazioni dell'Autorità Europea per la Sicurezza Alimentare (EFSA), secondo le quale il consumo giornaliero fino a $5 \mathrm{~g}$ di omega-3 è sicuro per la salute. ${ }^{108}$ In realtà la maggior parte delle evidenze scientifiche affermano che a tali dosaggi gli omega-3 non hanno evidenziato significativi benefici aggiuntivi nella prevenzione del cancro.

Una possibile interpretazione dell'apparente sovrautilizzo degli omega-3 deriva in parte dalla convenzione che al pari della loro nota efficacia nella prevenzione e nel trattamento delle malattie e dei fattori di rischio cardiovascolari, possono svolgere un ruolo efficace anche come molecole anti-cancro. Infatti, mentre per le malattie cardiovascolari, la dose di omega- 3 da assumere per ottenere reali benefici in termini di trattamento e prevenzione degli eventi coronarici maggiori è da tempo stabilita ed confermata anche dall'Agenzia Italiana del Farmaco (AIFA) circa la loro prescrivibilità e rimborsabilità, nel campo delle neoplasie la dose minima efficace per contrastare le diverse forme di cancro non è ancora stabilita. Tale concetto è applicabile anche negli USA dove per la prevenzione primaria delle malattie cardiovascolari, l'American Heart Association ${ }^{109}$ raccomanda per gli adulti in buona salute il consumo con la dieta di pesce, principale fonte di omega-3, almeno due volte la settimana. In modo ancora più definito l'Organizzazione Mondiale della Sanità raccomanda in prevenzione primaria, l'uso quotidiano EPA e DHA alla dose media di 0,3-0,5 g e un apporto di ALA di 0,8-1,1 g.

Al contrario la dose giornaliera utilizzata nei diversi studi per verificare la dose minima efficace in ambito di prevenzione e trattamento del cancro è molto variabile. In particolare, alcuni studi suggeriscono per il trattamento del cancro avanzato l'assunzione di 2,02-2,2 g di EPA e di 0,92-1,0 g di DHA per almeno cinque settimane. ${ }^{110}$ In altri l'assunzione orale giornaliera di 1,8 g di EPA 21 giorni prima e 180 giorni dopo un trapianto di midollo osseo si è dimostrata efficace nel miglioramento clinico della neoplasia ematologica. ${ }^{111}$ In uno studio Giapponese caso-controllo l'assunzione di olio di pesce contenente 3,1-3,2 $\mathrm{g}$ di EPA e 2,0-2,2 $\mathrm{g}$ di DHA ha ridotto l'incidenza di cancro del $64 \%{ }^{1{ }^{12}}$ Infine l'assunzione giornaliera di $5 \mathrm{~g}$ di olio di pesce che corrisponde a cinque capsule da $1 \mathrm{~g}$ contenenti ciascuna 200 mg di EPA e 367 mg di DHA, ha migliorato la prognosi del cancro prostatico. ${ }^{13}$

Vista l'ampia variabilità della dose di omega-3 testata nei diversi studi, prima di iniziare un trattamento con tali sostanze sarebbe opportuno attenersi alle conoscenze stabilite e condivise degli esperti del settore, anche se per una maggior definizione dovemmo attendere i risultati di alcuni trials di intervento in corso $^{114}$ con i quali si potrà probabilmente individuare la dose di omega-3 da assumere giornalmente per contrastare efficacemente la carcinogensi.

Vi è poi un ulteriore concetto non trascurabile che in parte spiega i diversi i risultati (a volte contrastanti) sul ruolo degli omega-3 su alcune neoplasie e che riguarda la loro bio-disponibilità. Infatti, negli ultimi anni numerose ricerche hanno evidenziato che a parità di dose di omega-3 assunti con la dieta o come supplementi/integratori, la loro effettiva bio-disponibilità - intesa ad esempio come concentrazione ematica ${ }^{115}$ è estremamente variabile da individuo ad individuo ed è in parte riconducibile all'età, a fattori ambientali e forse genetici. ${ }^{116,117}$

Un altro aspetto altrettanto importante riguarda non solo la quantità ma anche la qualità degli omega3 disponibili in commercio. ${ }^{118}$ Infatti, mentre per gli esperti del settore la prescrizione di prodotti contenenti omega-3 si avvale di conoscenze scientifiche e della personale esperienza acquisita nel corso degli anni, nei consumatori tout court vi è il rischio dell'uso di prodotti presenti in commercio con formulazioni e dosaggi diversi di EPA e DHA da quelli raccomandati e di qualità non sempre ottimali. D'altra parte, per ovviare il rischio dell'uso prodotti contenenti omega-3 poco efficaci, alcuni esperti consigliano di aumentare il consumo con la dieta di pesce, quest'ultimo principale fonte naturale di omega-3.

Tuttavia anche tale aspetto merita alcune considerazioni, tanto che alcuni ricercatori hanno evidenziato in numerosi studi la sempre più frequente contaminazione del pesce con agenti concerogeni può paradossalmente annullare i potenziali effetti preventivi sul cancro degli omega-3. ${ }^{117,119}$ A conferma dei dati della letteratura scientifica, recentemente l'Agenzia di sicurezza alimentare Francese e l'Enviromental Protection Agency Americana, hanno enfatizzato i rischi sulla salute derivanti dall'assunzione di pesce più di due volte la settimana a causa dell'inquinamento delle acque che contengono sempre maggiori concentrazioni di mercurio organico, policlorobifenili e diossina note sostanze ad azione tossica ed in particolare pro-cancerogena. ${ }^{120}$ Diventa quindi necessaria da parte non solo degli esperti del settore ma anche dell'intera opinione pubblica, una maggiore conoscenza sia della quantità che della qualità degli omega-3. In particolare per quest'ultima un gran merito va dato anche all'industria farmaceutica Italiana che negli ultimi anni - sulla base di precedenti trials di intervento ampiamente citati nella letteratura scientifica internazionale $\mathrm{e}^{41,42}$ - ha introdotto in commercio prodotti contenenti omega-3 di elevato livello quantitativo e qualitativo.

In conclusione, numerosi studi epidemiologici e clinici hanno ormai stabilito in modo inequivocabile che esiste una stretta associazione tra PUFA omega-3 e cancro. In particolare sono stati ampiamente definiti 
sia in modelli animali che in alcune neoplasie dell'uomo i meccanismi cellulari e molecolari attraverso i quali gli omega-3 modulano la carcinogenesi. Gli omega-3 si sono inoltre dimostrati efficaci nell'influenzare la prognosi clinica e la diffusione metastatica di alcune neoplasie aprendo nuovi scenari nell'ambito della ricerca oncologica dell'uomo. Non vi sono tuttavia evidenze scientifiche condivise sull'efficacia degli omega-3 nella prevenzione primaria del cancro, mentre non vi sono dubbi sulle loro capacità di essere ottimi coadiuvanti della terapia anti-neoplastica tradizionale, per la quale dovrà essere tuttavia definita la reale dose efficace. In particolare per rispondere a quest'ultimo quesito dovremo aspettare i risultati di importanti trials di intervento i cui dati saranno disponibili nei prossimi 2-3 anni. ${ }^{52,114,121-124}$

\section{Messaggio chiave}

I bias metodologici e procedurali, la diversa biodisponibiltà e qualità dei prodotti contenenti omega3 spiegano in gran parte $i$ dati contrastanti della letteratura circa la loro associazione con il cancro. La dose minima efficace ed i targets terapeutici da raggiungere dal trattamento con omega-3 per le diverse forme di cancro deve essere ancora stabilita e potrebbe essere svelata tra breve dai trials clinici di intervento in corso.

\section{Bibliografia}

1. Vineis P, Wild CP. Global cancer patterns: causes and prevention. Lancet 2014;383:549-57.

2. Kepp O, Senovilla L, Vitale I, et al.Consensus guidelines for the detection of immunogenic cell death. Oncoimmunology 2014;3:e955691.

3. Jansen RJ, Tan XL, Petersen GM. Gene-by-environment interactions in pancreatic cancer: implications for prevention. Yale J Biol Med 2015;88:115-26.

4. Gregor O, Toman R, Prusová F. Gastrointestinal cancer and nutrition. Gut 1969;10:1031-4.

5. Hormozdiari H, Day NE, Aramesh B, et al. Dietary factors and esophageal cancer in the Caspian Littoral of Iran. Cancer Res 1975;35:3493-8.

6. Oiso T. Incidence of stomach cancer and its relation to dietary habits and nutrition in Japan between 1900 and 1975. Cancer Res 1975;35:3254-8.

7. Alcantara EN, Speckmann EW. Diet, nutrition, and cancer. Am J Clin Nutr 1976;29:1035-47.

8. Rogers AE, Newberne PM. Dietary effects on chemical carcinogenesis in animal models for colon and liver tumors. Cancer Res 1975;35:3427-31.

9. Lin PH, Aronson W, Freedland SJ. Nutrition, dietary interventions and prostate cancer: the latest evidence. BMC Med 2015;13:3.

10. Bracken CP, Khew-Goodall Y, Goodall GJ. Networkbased approaches to understand the roles of miR-200 and other microRNAs in Cancer. Cancer Res 2015 [Epub ahead of print].
11. zur Hausen H. Red meat consumption and cancer: reasons to suspect involvement of bovine infectious factors in colorectal cancer. Int J Cancer 2012;130:2475-83.

12. Balaban S, Lee LS, Schreuder M, et al. Obesity and cancer progression: is there a role of fatty acid metabolism? Biomed Res Int 2015;2015:274585.

13. Salaspuro M. Interactions of alcohol and tobacco in gastrointestinal cancer. J Gastroenterol Hepatol 2012; 27:135-9.

14. Ostan R, Lanzarini C, Pini E, et al. Inflammaging and cancer: a challenge for the Mediterranean diet. Nutrients 2015;7:2589-621.

15. Abel S, Riedel S, Gelderblom WC. Dietary PUFA and cancer. Proc Nutr Soc 2014;73:361-7.

16. Harris WS, Bulchandani D. Why do omega-3 fatty acids lower serum triglycerides? Curr Opin Lipidol 2006; 17:387.

17. Freund-Levi Y, Vedin I, Hjorth E, et al. Effects of Supplementation with omega-3 fatty acids on oxidative stress and inflammation in patients with Alzheimer's disease: the OmegAD study. J Alzheimers Dis 2014;42: 823-31.

18. Patterson WL 3rd, Georgel PT. Breaking the cycle: the role of omega-3 polyunsaturated fatty acids in inflammation-driven cancers. Biochem Cell Biol 2014;92: 321-8.

19. Isobe $Y$, Arita M. Identification of novel omega- 3 fatty acid-derived bioactive metabolites based on a targeted lipidomics approach. J Clin Biochem Nutr 2014;55: 79-84.

20. Superko HR, Superko AR, Lundberg GP, et al. Omega3 fatty acid blood levels clinical significance update. Curr Cardiovasc Risk Rep 2014;8:407.

21. Chua ME, Sio MC, Sorongon M, et al. The relevance of serum levels of long chain omega-3 polyunsaturated fatty acids and prostate cancer risk: a meta-analysis. Can Urol Assoc J 2013;7:E333-43.

22. Superko HR, Superko SM, Nasir K, et al. Omega-3 fatty acid blood levels: clinical significance and controversy. Circulation 2013;128:2154-61.

23. De Oliveira Otto MC, Wu JH, Baylin A, et al. Circulating and dietary omega-3 and omega- 6 polyunsaturated fatty acids and incidence of CVD in the multi-ethnic study of atherosclerosis. J Am Heart Assoc 2013;2: e000506.

24. Moltó-Puigmartí FC, Plat J, Mensink RP, et al. FADS1 FADS2 gene variants modify the association between fish intake and the docosahexaenoic acid proportions in human milk. Am J Clin Nutr 2010;91:1368-76.

25. Russo GL. Dietary n-6 and n-3 polyunsaturated fatty acids: from biochemistry to clinical implications in cardiovascular prevention. Biochem Pharmacol 2009;77: 937-46.

26. Dyall SC. Long-chain omega-3 fatty acids and the brain: a review of the independent and shared effects of EPA, DPA and DHA. Front Aging Neurosci 2015 21;7:52.

27. Block RC, Abdolahi A, Tu X, et al. The effects of aspirin on platelet function and lysophosphatidic acids depend on plasma concentrations of EPA and DHA. Prostaglandins Leukot Essent Fatty Acids 2015;96:17-24.

28. Nakamura H, Murayama T. Role of sphingolipids in arachidonic acid metabolism. J Pharmacol Sci 2014; 124:307-12. 
29. Di Nicolantonio JJ, McCarty MF, Chatterjee S, et al. A higher dietary ratio of long-chain omega-3 to total omega- 6 fatty acids for prevention of COX-2-dependent adenocarcinomas. Nutr Cancer 2014;66:1279-84.

30. Simopoulos AP. Omega-6/omega-3 essential fatty acids: biological effects. World Rev Nutr Diet 2009; 99:1-16.

31. Binukumar B, Mathew A. Dietary fat and risk of breast cancer. World J Surg Oncol 2005;3:45.

32. Spencer L, Mann C, Metcalfe M, et al. The effect of omega-3 FAs on tumour angiogenesis and their therapeutic potential. Eur J Cancer 2009;45:2077-86.

33. Bartsch H, Nair J, Owen RW. Dietary polyunsaturated fatty acids and cancers of the breast and colorectum: emerging evidence for their role as risk modifiers. Carcinogenesis 1999;20:2209-18.

34. Granados S, Quiles JL, Gil A et al. Lípidos de la dieta y cáncer. Nutr Hosp 2006;21:44-54.

35. Pizato N, Bonatto S, Yamazaki RK, et al. Ratio of n6 to n-3 fatty acids in the diet affects tumor growth and cachexia in Walker 256 tumor-bearing rats. Nutr Cancer 2005;53:194-201.

36. Olivo SE, Hilakivi-Clarke L. Opposing effects of prepubertal low- and high-fat n-3 polyunsaturated fatty acid diets on rat mammary tumorigenesis. Carcinogenesis 2005;26:1563-72.

37. Goodstine SL, Zheng T, Holford TR, et al. Dietary ( $\omega$ $3) /(\omega-6)$ fatty acid ratio: possible relationship to premenopausal but not to post-menopausal breast cancer risk in US women. J Nutr 2003;133:1409-14.

38. Maillard V, Bougoux P, Ferrari P, et al. n-3 and n-6 fatty acids in breast adipose tissue and relative risk of breast cancer in a case control study in Tours, France. Int J Cancer 2002;98:78-83.

39. Chapkin RS, Seo J, McMurray DN, et al. Mechanisms by which docosahexaenoic acid and related fatty acids reduce colon cancer risk and inflammatory disorders of the intestine. Chem Phys Lipids 2008;153:14-23.

40. Chajès V, Bougnoux P. Omega-6/omega-3 polyunsaturated fatty acid ratio and cancer. World Rev Nutr Diet 2003;92:133-51.

41. Andreeva VA, Touvier M, Kesse-Guyot E, et al. B vitamin and/or $\omega-3$ fatty acid supplementation and cancer: ancillary findings from the supplementation with folate, vitamins B6 and B12, and/or omega-3 fatty acids (SU.FOL.OM3) randomized trial. Arch Intern Med 2012;172:540.

42. [No authors listed]. Dietary supplementation with n-3 polyunsaturated fatty acids and vitamin $\mathrm{E}$ after myocardial infarction: results of the GISSI-Prevenzione trial. Gruppo Italiano per lo Studio della Sopravvivenza nell'Infarto miocardico. Lancet 1999;354:447-55.

43. Yokoyama M, Origasa H, Matsuzaki M, et al. Effects of eicosapentaenoic acid on major coronary events in hypercholesterolaemic patients (JELIS): a randomised open-label, blinded endpoint analysis. Lancet 2007; 369:1090.

44. Wendel M, Heller AR. Anticancer actions of omega-3 fatty acids-current state and future perspectives. Anticancer Agents Med Chem 2009;9:457.

45. Wiseman M. The second World Cancer Research Fund/American Institute for Cancer Research expert report. Food, nutrition, physical activity, and the preven- tion of cancer: a global perspective. Proc Nutr Soc 2008;67:253.

46. Sala-Vila A, Calder PC. Update on the relationship of fish intake with prostate, breast, and colorectal cancers. Crit Rev Food Sci Nutr 2011;51:855.

47. Cockbain AJ, Toogood GJ, Hull MA. Omega-3 polyunsaturated fatty acids for the treatment and prevention of colorectal cancer. Gut 2012;61:135.

48. Zheng JS, Hu XJ, Zhao YM, et al. Intake of fish and marine n-3 polyunsaturated fatty acids and risk of breast cancer: meta-analysis of data from 21 independent prospective cohort studies. BMJ 2013;346:f3706.

49. Brasky TM, Till C, White E, et al. Serum phospholipid fatty acids and prostate cancer risk: results from the prostate cancer prevention trial. Am J Epidemiol 2011;173:1429.

50. Brasky TM, Darke AK, Song X, et al. Plasma phospholipid fatty acids and prostate cancer risk in the SELECT trial. J Natl Cancer Inst 2013;105:1132.

51. Chavarro JE, Stampfer MJ, Li H, et al. A prospective study of polyunsaturated fatty acid levels in blood and prostate cancer risk. Cancer Epidemiol Biomarkers Prev 2007;16:1364.

52. Hull MA, Sandell AC, Montgomery AA, et al. A randomized controlled trial of eicosapentaenoic acid and/or aspirin for colorectal adenoma prevention during colonoscopic surveillance in the NHS Bowel Cancer Screening Programme (The seAFOod Polyp Prevention Trial): study protocol for a randomized controlled trial. Trials. 2013;14:237

53. Attard G, Parker C, Eeles RA, et al. Prostate cancer. Lancet 2015;14:61947-4.

54. Sander M, Oxlund B, Jespersen A, et al. The challenges of human population ageing. Age Ageing 2015;44:185-7.

55. Bishop KS, Erdrich S, Karunasinghe N, et al. An investigation into the association between DNA damage and dietary fatty acid in men with prostate cancer. Nutrients 2015;7:405-22.

56. Simopoulos AP, Leaf A, Salem N Jr. workshop on the essentiality of and recommended dietary intakes for omega-6 and omega-3 fatty acids. J Am Coll Nutr 1999; 18:487-9.

57. Fradet V, Cheng I, Casey G, et al. Dietary omega- 3 fatty acids, cyclooxygenase- 2 genetic variation, and aggressive prostate cancer risk. Clin Cancer Res 2009;15: 2559-66.

58. Alexander DD, Bassett JK, Weed DL, et al. Metaanalysis of long-chain omega-3 polyunsaturated fatty acids (LC $\omega$-3PUFA) and prostate cancer. Nutr Cancer 2015; 67:543-54.

59. Di Nicolantonio JJ, McCarty MF, Lavie CJ, et al. Do omega-3 fatty acids cause prostate cancer? Mo Med 2013;110:293-5.

60. Liu Z, Hopkins MM, Zhang Z, et al. Omega-3 fatty acids and other FFA4 agonists inhibit growth factor signaling in human prostate cancer cells. J Pharmacol Exp Ther 2015;352:380-94.

61. Alexander DD, Bassett JK, Weed DL, et al. Metaanalysis of long-chain omega-3 polyunsaturated fatty acids (LC $\omega$-3PUFA) and prostate cancer. Nutr Cancer 2015; 67:543-54.

62. Jemal A, Bray F, Center MM, et al. Global cancer statistics. CA Cancer J Clin 2011;61:69-90. 
63. Brennan SF, Cantwell MM, Cardwell CR, et al. Dietary patterns and breast cancer risk: a systematic review and meta-analysis. Am J Clin Nutr 2010;91:1294-302.

64. Lambrechts S, Decloedt J, Neven P. Breast cancer prevention: lifestyle changes and chemoprevention. Acta Clin Belg 2011;66:283-92.

65. Saadatian-Elahi M, Norat T, Goudable J, et al. Biomarkers of dietary fatty acid intake and the risk of breast cancer: a meta-analysis. Int J Cancer 2004;111: 584-91.

66. Wakai K, Tamakoshi K, Date C, et al. Dietary intakes of fat and fatty acids and risk of breast cancer: a prospective study in Japan. Cancer Sci 2005;96:590-9.

67. Kim EH, Willett WC, Colditz GA, et al. Dietary fat and risk of postmenopausal breast cancer in a 20-year follow-up. Am J Epidemiol 2006;164:990-7.

68. MacLean CH, Newberry SJ, Mojica WA, et al. Effects of omega-3 fatty acids on cancer risk: a systematic review. JAMA 2006;295:403-15.

69. Park SY, Kolonel LN, Henderson BE, et al. Dietary fat and breast cancer in postmenopausal women according to ethnicity and hormone receptor status: the Multiethnic Cohort Study. Cancer Prev Res (Phila) 2012;5:216-28.

70. Karmali RA, Marsh J, Fuchs C. Effect of omega-3 fatty acids on growth of a rat mammary tumor. J Natl Cancer Inst 1984;73:457-61.

71. Kort WJ, Weijma IM, Bijma AM, et al. Omega-3 fatty acids inhibiting the growth of a transplantable rat mammary adenocarcinoma. J Natl Cancer Inst 1987;79: 593-9.

72. Schley PD, Jijon HB, Robinson LE, et al. Mechanisms of omega-3 fatty acid-induced growth inhibition in MDA-MB-231 human breast cancer cells. Breast Cancer Res Treat 2005;92:187-95.

73. Gago-Dominguez M, Yuan JM, Sun CL, et al. Opposing effects of dietary n-3 and n- 6 fatty acids on mammary carcinogenesis: the Singapore Chinese Health Study. Br J Cancer 2003;89:1686-92.

74. Jiang W, Zhu Z, McGinley JN, et al. Identification of a molecular signature underlying inhibition of mammary carcinoma growth by dietary N-3 fatty acids. Cancer Res 2012;72:3795-806.

75. Manni A, Richie Jr JP, Xu H, et al. Effects of fish oil and tamoxifen on preneoplastic lesion development and biomarkers of oxidative stress in the early stages of $\mathrm{N}$ methyl-N-nitrosoureainduced rat mammary carcinogenesis. Int J Oncol 2011;39:1153-64.

76. Cho E, Spiegelman D, Hunter DJ, et al. Premenopausal fat intake and risk of breast cancer. J Natl Cancer Inst 2003;95:1079-85.

77. Folsom AR, Demissie Z. Fish intake, marine omega-3 fatty acids, and mortality in a cohort of postmenopausal women. Am J Epidemiol 2004;160:1005-10.

78. Holmes MD, Colditz GA, Hunter DJ, et al. Meat, fish and egg intake and risk of breast cancer. Int J Cancer 2003;104:221-7.

79. Engeset D, Alsaker E, Lund E, et al. Fish consumption and breast cancer risk. The European Prospective Investigation into Cancer and Nutrition (EPIC). Int J Cancer 2006;119:175-82.

80. Zheng JS, Hu XJ, Zhao YM, et al. Intake of fish and marine n-3 polyunsaturated fatty acids and risk of breast cancer: meta-analysis of data from 21 independ- ent prospective cohort studies. BMJ 2013;346:f3706.

81. Wang L, Chen J, Thompson LU. The inhibitory effect of flaxseed on the growth and metastasis of estrogen receptor negative human breast cancer xenograftsis attributed to both its lignan and oil components. Int $\mathrm{J}$ Cancer 2005; 116:793-8.

82. Hilakivi-Clarke L, Cho E, Cabanes A, et al. Dietary modulation of pregnancy estrogen levels and breast cancer risk among female rat offspring. Clin Cancer Res 2002;8:3601-10.

83. Bougnoux P, Hajjaji N, Ferrasson MN, et al. Improving outcome of chemotherapy of metastatic breast cancer by docosahexaenoic acid: a phase II trial. Br J Cancer 2009;101:1978-85.

84. Jemal A, Siegel R, Xu J, Ward E. Cancer statistics, 2010. CA Cancer J Clin 2010;60:277-300.

85. Cai Q, Gao YT, Chow WH, et al. Prospective study of urinary prostaglandin E2 metabolite and colorectal cancer risk. J Clin Oncol 2006;24:5010-6.

86. Schottenfeld D, Beebe-Dimmer J. Chronic inflammation: a common and important factor in the pathogenesis of neoplasia. CA Cancer J Clin 2006;56:69-83.

87. Ebrahimi M, Ghayour-Mobarhan M, Rezaiean S. Omega-3 fatty acid supplements improve the cardiovascular risk profile of subjects with metabolic syndrome, including markers of inflammation and auto-immunity. Acta Cardiol 2009;64:321-7.

88. Micallef MA, Garg ML. Anti-inflammatory and cardioprotective effects of $n-3$ polyunsaturated fatty acids and plant sterols in hyperlipidemic individuals. Atherosclerosis 2009;204:476-82.

89. Schloss I, Kidd MS, Tichelaar HY, et al. Dietary factors associated with a low risk of colon cancer in coloured west coast fishermen. S Afr Med J 1997;87:152-8.

90. Calviello G, Serini S, Piccioni E. n-3 polyunsaturated fatty acids and the prevention of colorectal cancer: molecular mechanisms involved. Curr Med Chem 2007; 14:3059-69.

91. Habbel P, Weylandt KH, Lichopoj K, et al. Docosahexaenoic acid suppresses arachidonic acid-induced proliferation of LS-174T human colon carcinoma cells. World J Gastroenterol 2009;15:1079-84.

92. Kantor ED, Lampe JW, Peters U, et al. Long-chain omega-3 polyunsaturated fatty acid intake and risk of colorectal cancer. Nutr Cancer 2014;66:716-27.

93. Kliewer SA, Willson TM. The nuclear receptor PPAR $\gamma$ - bigger than fat. Curr Opin Genet Dev 1998;8:576-81.

94. Trombetta A, Maggiora M, Martinasso G, et al. Arachidonic and docosahexaenoic acids reduce the growth of A549 human lung-tumor cells increasing lipid peroxidation and PPARs. Chem Biol Interact 2007;165:239-50.

95. Han S, Sun X, Ritzenthaler JD, Roman J. Fish oil inhibits human lung carcinoma cell growth by suppressing integrin-linked kinase. Mol Cancer Res 2009;7: 108-17.

96. Yang P, Cartwright C, Chan D, et al. Anticancer activity of fish oils against human lung cancer is associated with changes in formation of PGE2 and PGE3 and alteration of Akt phosphorylation. Mol Carcinog 2014;53:566-77.

97. van der Meij BS, Langius JA, Spreeuwenberg MD, et al. Oral nutritional supplements containing n-3 polyunsaturated fatty acids affect quality of life and functional status in lung cancer patients during multimodality 
treatment: an RCT. Eur J Clin Nutr 2012;66:399-404.

98. Barber MD, Ross JA, Voss AC, et al. The effect of an oral nutritional supplement enriched with fish oil on weight-loss in patients with pancreatic cancer. $\mathrm{Br} \mathrm{J}$ Cancer 1999;81:80-6.

99. de Aguiar Pastore Silva J, Emilia de Souza Fabre M, Waitzberg DL. Omega-3 supplements for patients in chemotherapy and/or radiotherapy: a systematic review. Clin Nutr 2015;34:359-66.

100. Manni A, Richie JP Jr, Xu H, Washington S, et al. Influence of omega-3 fatty acids on Tamoxifen-induced suppression of rat mammary carcinogenesis. Int J Cancer 2014;134:1549-57.

101. Wynter MP, Russell ST, Tisdale MJ. Effect of n-3 fatty acids on the antitumour effects of cytotoxic drugs. In vivo 2004;18:543-7.

102. Zhang G, Panigrahy D, Mahakian LM, et al. Epoxy metabolites of docosahexaenoic acid (DHA) inhibit angiogenesis, tumor growth, and metastasis. Proc Natl Acad Sci U S A 2013;110:6530-5.

103. Taguchi A, Kawana K, Tomio K, et al. Matrix metalloproteinase (MMP)-9 in cancer-associated fibroblasts (CAFs) is suppressed by omega-3 polyunsaturated fatty acids in vitro and in vivo. PLoS One 2014;9:e89605.

104. Liu XH, Rose DP. Suppression of type IV collagenase in MDA-MB-435 human breast cancer cells by eicosapentaenoic acid in vitro and in vivo. Cancer Lett 1995;92:21-6.

105. Mandal CC, Ghosh-Choudhury T, Yoneda T, et al. Fish oil prevents breast cancer cell metastasis to bone. Biochem Biophys Res Commun 2010;402:602-7.

106. Chen Z, Zhang Y, Jia C, et al. mTORC1/2 targeted by n-3 polyunsaturated fatty acids in the prevention of mammary tumorigenesis and tumor progression. Oncogene 2014;33:4548-57.

107. Food Ingredients 1st. DSM Nutritional products. Available from: http://www.foodingredientsfirst.com/supplier-profile/DSM-Nutritional-Products.html

108. EFSA Panel on Dietetic Products, Nutrition and Allergies (NDA). Scientific opinion on the tolerable upper intake level of eicosapentaenoic acid (EPA), docosahexaenoic acid (DHA) and docosapentaenoic acid (DPA). EFSA Journal 2012;10:2815.

109. Eckel RH, Jakicic JM, Ard JD, et al. American College of Cardiology/American Heart Association Task Force on Practice Guidelines 2013 AHA/ACC guideline on lifestyle management to reduce cardiovascular risk: a report of the American College of Cardiology/American Heart Association Task Force on Practice Guidelines. J Am Coll Cardiol 2014;63:2960-84.

110. Ries A, Trottenberg P, Elsner F, et al. A systematic review on the role of fish oil for the treatment of cachexia in advanced cancer: an EPCRC cachexia guidelines project. Palliat Med 2012;26:294-304.

111. Roeland E, Mitchell W, Elia G, et al. Symptom control in stem cell transplantation: a multidisciplinary palliative care team approach. Part 2: psychosocial concerns. J Support Oncol 2010;8:179-83.

112. Kuriki K. Risk of colorectal cancer is linked to erythrocyte compositions of fatty acids as biomarkers for dietary intakes of fish, fat, and fatty acids. Cancer Epidemiol Biomarkers Prev 2006;15:1791-8.
113. Brasky TM, Darke AK, Song X, et al. Plasma phospholipid fatty acids and prostate cancer risk in the SELECT Trial. J Natl Cancer Inst 2013;105:1132-820.

114. LeBoff MS, Yue AY, Copeland T, et al. VITAL-Bone Health: rationale and design of two ancillary studies evaluating the effects of vitamin D and/or omega-3 fatty acid supplements on incident fractures and bone health outcomes in the VITamin D and OmegA-3 TriaL (VITAL). Contemp Clin Trials 2015;41:259-68.

115. Pounis G, de Lorgeril M, Salen P, et al. European Collaborative Group of the IMMIDIET Project. Dietary patterns and fatty acids levels of three European populations. Results from the IMMIDIET study. Nutr Metab Cardiovasc Dis 2014;24:883-90.

116. Risé P, Tragni E, Ghezzi S, et al. Different patterns characterize Omega 6 and Omega 3 long chain polyunsaturated fatty acid levels in blood from Italian infants, children, adults and elderly. Prostaglandins Leukot Essent Fatty Acids 2013;89:215-20.

117. Cicero AF, Morbini M, Borghi C. Do we need 'new' omega- 3 polyunsaturated fatty acids formulations? Expert Opin Pharmacother 2015;16:285-8

118. Hoekstra J, Hart A, Owen H, et al. Fish, contaminants and human health: quantifying and weighing benefits and risks. Food Chem Toxicol 2013;54:18-29.

119. Mozaffarian D, Rimm EB. Fish intake, contaminants, and human health: evaluating the risks and the benefits. JAMA 2006;296:1885-99.

120. U.S. Environmental Protection Agency. Trends in blood mercury concentrations and fish consumption among U.S. women of childbearing age NHANES, 1999-2010. Final Report, July 2013. EPA-823-R-13-002. Disponibile su: http:/water.epa.gov/scitech/swguidance/fishshellfish/fishadvisories/upload/Trends-in-Blood-Mercu ry-Concentrations-and-Fish-Consumption-Among-US-Women-of-Childbearing-Age-NHANES-19992010.pdf

121. Viecelli AK, Pascoe E, Polkinghorne KR, et al. FAVOURED study team. The Omega-3 fatty acids (Fish Oils) and Aspirin in Vascular access OUtcomes in REnal Disease (FAVOURED) study: the updated final trial protocol and rationale of post-initiation trial modifications. BMC Nephrol 2015;16:89.

122. Hershman DL, Unger JM, Crew KD, et al. randomized multicenter placebo-controlled trial of omega- 3 fatty acids for the control of aromatase inhibitor-induced musculoskeletal pain: SWOG S0927. J Clin Oncol 2015;33:1910-7.

123. Pottel L, Lycke M, Boterberg T, et al. Echium oil is not protective against weight loss in head and neck cancer patients undergoing curative radio(chemo) therapy: a randomised-controlled trial. BMC Complement Altern Med 2014;14:382.

124. Sorensen LS, Thorlacius-Ussing O, Rasmussen HH, et al. Effects of perioperative supplementation with omega-3 fatty acids on leukotriene B and leukotriene $B$ production by stimulated neutrophils in patients with colorectal cancer: a randomized, placebo-controlled intervention trial. Nutrients 2014;6:4043-57. 
Farmaci nella forma migliore

\title{
Gli omega-3: trigliceridi naturali o esteri etilici?
}

\author{
Paola Zuccheri, Corrado Iacono, Giulia Benini
}

Dipartimento Farmaceutico, AUSL Bologna, Italia

\section{Introduzione}

Gli omega-3, conosciuti anche con il nome di PUFA n-3 (polyunsaturated fatty acids), sono una categoria di acidi grassi polinsaturi essenziali (Figura 1). Le categorie di omega-3 coinvolte nella fisiologia umana sono l'acido $\alpha$-linolenico (ALA), l'acido eicosapentaenoico (EPA) e l'acido docosaesaenoico (DHA). Per le principali caratteristiche strutturali e aspetti funzionali si rimanda ai paragrafi precedenti.

Le due serie omega-6 e -3 includono un composto precursore a 18 atomi di carbonio (PUFA a catena corta), rispettivamente l'acido linoleico (AL) e ALA le cui fonti primarie in natura sono costituite da grassi (oli) vegetali sintetizzati esclusivamente nelle piante. Gli animali che si nutrono di tali piante possono indirettamente contenere tali precursori. Nell'organismo AL e ALA sono convertiti a PUFA a lunga catena (EPA e DHA) attraverso reazioni enzimatiche che però risultano inefficienti, pertanto tali composti, in particolare il DHA, devono venire assunti già preformati con la dieta o con integratori.

\section{Le diverse formulazioni degli omega-3: esteri etilici e trigliceridi}

I processi per ottenere l'olio di pesce crudo sono numerosi e spaziano dalle fasi tradizionali di estrazione dalla materia prima per semplice riscaldamento e pressione, all'uso della tecnica di estrazione attraverso fluidi supercritici. Alla fase di estrazione segue

Corrispondente: Paola Zuccheri, UOC Farmacia Clinica dei Blocchi operatori, Dipartimento Farmaceutico AUSL di Bologna, Ospedale Maggiore, Largo Nigrisoli 2, 40133 Bologna, Italy.

Tel.: +39.051.3172166

E-mail: paola.zuccheri@ausl.bologna.it

Articolo pubblicato secondo la Creative Commons Attribution NonCommercial 3.0 License (CC BY-NC 3.0).

CCopyright P. Zuccheri et al., 2015

Licensee PAGEPress, Italy

QUADERNI - Italian Journal of Medicine 2015; 3:301-305 quella di raffinazione in quanto l'estratto è costituito da una miscela composta da acidi grassi liberi, gliceridi, fosfolipidi, steroli, pigmenti e, a volte, anche metalli pesanti e tossici (es. mercurio)

I prodotti oggi in commercio si presentano come esteri etilici (EE) o trigliceridi (TG) e differiscono dall'estratto crudo dell'olio di pesce perché sono facilmente metabolizzabili dal corpo umano e con una buona stabilità contro l'ossidazione.

Sia i TG che gli EE sono forme esterificate ovvero gli acidi grassi nei TG sono esterificati al glicerolo mentre gli acidi grassi negli EE sono esterificati ad etanolo. EPA e DHA in forma di EE sono acidi grassi altamente raffinati che vengono prodotti per reazione dagli acidi grassi liberi (FFA) derivati dalla raffinazione dell'olio di pesce crudo con etanolo, in un processo chiamato trans-esterificazione. Questo processo comporta la rimozione della catena principale di glicerolo nella forma TG dell'olio di pesce, con conseguente rilascio di FFA e glicerolo. Una molecola di etanolo è poi collegata agli FFA, creando EE.

A questo processo segue una distillazione che permette la concentrazione selettiva di EPA e DHA (nella forma etil-EPA ed etil-DHA) in livelli maggiori rispetto a quelle naturalmente presenti nel pesce, ovvero circa $50-70 \%$.

Il prodotto di partenza per sintetizzare omega-3 sotto forma di TG naturali è sempre l'olio di pesce crudo che viene convertito in EE e successivamente in forma TG, utilizzando enzimi in un processo chiamato glicerolisi. Questi enzimi separano la molecola di etanolo dagli acidi grassi con conseguente produzione degli FFA e una molecola di etanolo. Quando a questo processo si aggiunge di nuovo glicerolo nella soluzione, gli enzimi ri-esterificano gli acidi grassi sul glicerolo creando, dopo una nuova fase di distillazione, oli di TG. Questi oli sono comunemente noti come trigliceridi riformati o ri-esterificati (RTG), con strutture identiche a quelle naturali TG ma con concentrazioni elevate di acidi grassi EPA e DHA.

Il passaggio per arrivare dalla forma $\mathrm{EE}$ a quella RTG implica un incremento dei costi del 50- 80\% rispetto alla semplice produzione di EE, pertanto la maggior parte degli omega-3 in commercio è sottoforma di EE. Gli omega-3 in forma liquida sono prodotti principalmente come TG in quanto gli EE liquidi 
si degradano velocemente e hanno un sapore più intenso di pesce e quindi risultano sgradevoli al gusto e all'olfatto.

Diversi gruppi di ricercatori hanno dimostrato una migliore biodisponibilità e metabolismo nella conversione di olio di pesce da EE concentrati a TG naturale. ${ }^{1}$

A conferma di questa teoria, un gruppo di ricercatori guidato da J. Schuchardt della Leibniz Universitat di Hannover (Germania), ha condotto uno studio ${ }^{2}$ in pazienti affetti da dislipidemie. I risultati hanno evidenziato la superiorità nell'assunzione di omega-3 EPA e DHA nella forma TG rispetto a quella EE.

Un'altra ricerca condotta da Schuchardt e colleghi pubblicata sulla rivista European Journal of Clinical Nutrition nel $2010,{ }^{3}$ ha inoltre dimostrato che la concentrazione di omega-3 EPA e DHA rilevabile nel sangue umano dopo l'assunzione di integratori di omega-3, aumenta del $50 \%$ proprio se i principi attivi sono assunti sotto forma di TG.

Durante questo studio sono stati selezionati 150 soggetti divisi in tre gruppi ma accumunati da livelli anomali di grassi nel sangue [elevato colesterolo con lipoproteine a bassa densità (LDL) e basso colesterolo con lipoproteine ad alta densità] in terapia farmacologica ipocolesterolomizzante. Ad un gruppo è stato somministrato un integratore placebo con olio di grano, al secondo gruppo un integratore alimentare di omega-3 EPA e DHA in forma TG ed al terzo gruppo un integratore di omega-3 EPA e DHA in forma EE.

I risultati della ricerca hanno evidenziato che nell'organismo i livelli degli acidi grassi EPA e DHA aumentano di più e più rapidamente se gli omega-3 vengono assunti sotto forma di TG. L'aumento degli acidi grassi liberi e circolanti nel sangue è risultato invece inferiore se gli omega-3 EPA e DHA sono assunti in forma di EE.

Non ultimo l'assunzione di integratori alimentari di omega-3 nella forma di trigliceridi riesterificati RTG, contribuisce in modo significativo a ridurre i livelli ematici di lipidi LDL. Inoltre la forma RTG è più facilmente assorbita e meglio sfruttata dall'organismo come dimostrato da Jorn Dyerberg ${ }^{4} \mathrm{e}$ colleghi in studi condotti presso l'Università di Copenhagen e dell'Ospedale di Aalborg (Danimarca).

I ricercatori hanno evidenziato come l'assorbimento e la concentrazione plasmatica di omega-3 EPA e DHA presenti nell'integratori alimentari assunti sotto forma di RTG, è superiore del $50 \%$ rispetto alle altre forme di omega-3 EE. In totale sono stati coinvolti 72 individui di età compresa tra i 21 e i 56 anni. Ciascun partecipante ha ricevuto per 2 settimane una dose giornaliera di omega-3 EPA e DHA compresa tra 3,1 e 3,6 g. Al termine della sperimentazione, i ricercatori hanno rilevato che la biodisponibilità degli

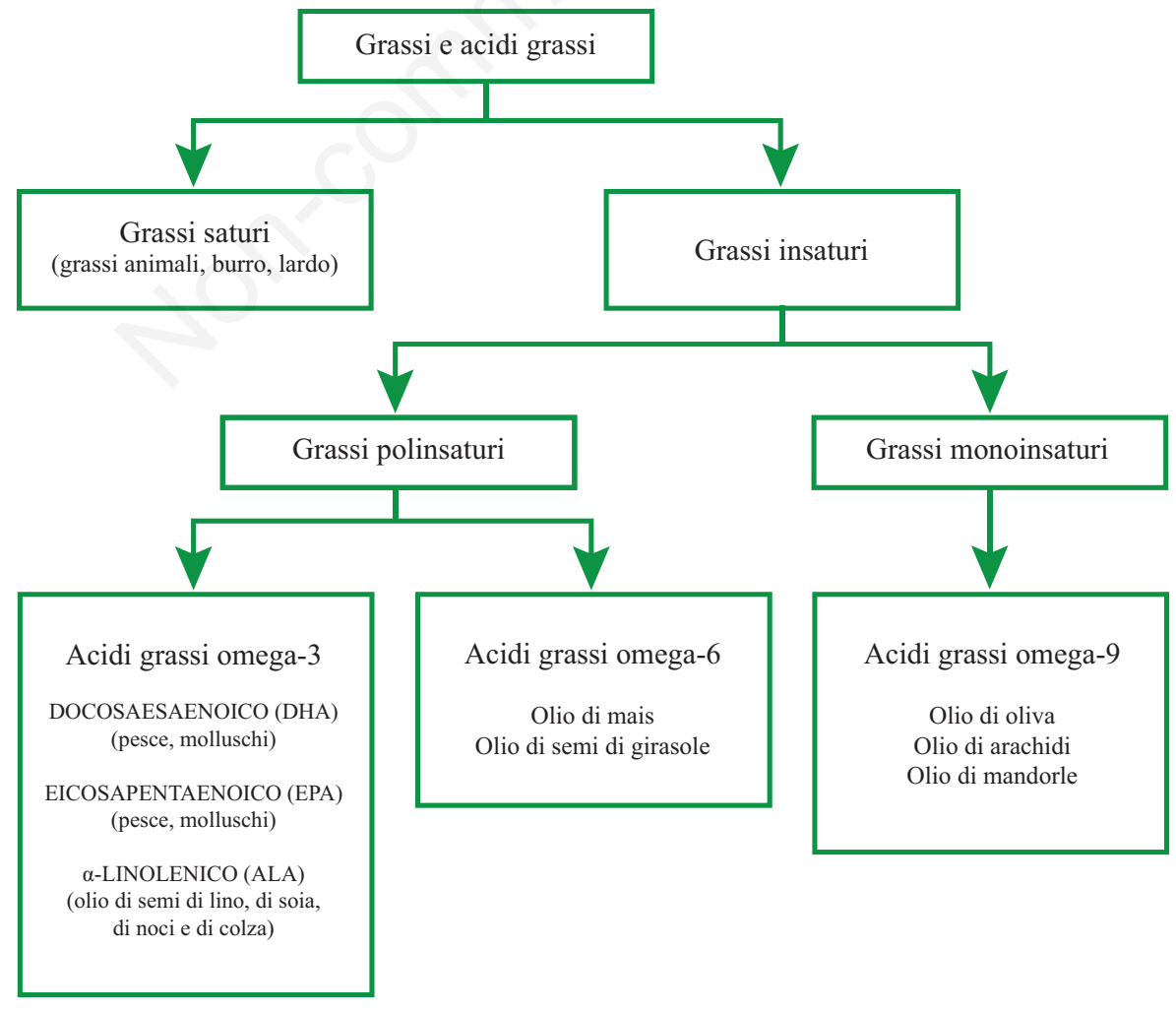

Figura 1. Classificazione dei grassi e acidi grassi. 
omega-3 era del 73\% nel caso degli EE, del 91\% nel caso degli acidi grassi liberi TG e del $124 \%$ nel caso dei trigliceridi riesterificati RTG.

\section{Farmacocinetica}

L'assorbimento degli omega-3 in forma EE e TG avviene per la maggior parte nell'intestino tenue e se l'assunzione avviene a stomaco pieno dopo un pasto ad alto contenuto di grassi, la biodisponibilità aumenta fino a 80 volte rispetto all'assunzione a stomaco vuoto. $^{5}$

Indifferentemente dalla formulazione chimica, l'EPA sembra accumularsi nel sangue con delle concentrazioni direttamente proporzionali alle quantità di omega-3 assunte, mentre il DHA si accumula alle stesse concentrazioni indifferentemente dalle quantità ingerite. ${ }^{6} \mathrm{Nel}$ tratto gastrointestinale la lipasi pancreatica, secreta come prolipasi dai dotti pancreatici e attivata dai sali biliari e dalla colipasi, idrolizza gli EE e i TG in un processo chiamato lipolisi, generando etanolo o glicerolo e liberando EPA e DHA sotto forma di FFA. Il passaggio nelle cellule della mucosa del digiuno avviene per semplice diffusione. All'interno delle cellule gli FFA vengono risintetizzati in TG e formano i chilomicroni. Sono questi gli aggregati molecolari capaci di migrare nel sangue e nei vasi linfatici. Riprendendo i risultati sulla biodisponibilità valutati da Dyerberg 4 (olio di pesce crudo 100\%, RTG 124\%, EE 73\% e FFA 91\%), la minore biodisponibilità di EE rispetto a RTG potrebbe essere dovuta in parte al fatto che gli enzimi dediti alla digestione dei grassi sono meno efficaci a rompere i legami di un EE rispetto ai legami della struttura RTG. ${ }^{7-9}$

Tutti i grassi alimentari sono digeriti nel piccolo intestino dall'azione di acidi biliari e lipasi pancreatica.

Per quanto riguarda gli omega-3 sottoforma di TG, gli acidi biliari rompono i globuli TG in goccioline di emulsione molto più piccole, che aumentano la superficie e qui le lipasi pancreatiche possono liberare due dei tre acidi grassi dal TG, creando quindi due FFA e un monogliceride (un acido grasso attaccato ancora al glicerolo). FFA e il monogliceride poi formano micelle che vengono assorbite grazie agli enterociti intestinali. Una volta all'interno dell'enterocita, la FFA e il monogliceride viene riassemblato di nuovo in TG. Questi TG insieme ad altre molecole formano i chilomicroni che sono la forma di trasporto principale dei TG per giungere dal canale linfatico al fegato. ${ }^{9}$

La digestione degli EE è leggermente diversa perché sono privi della catena principale di glicerolo. Nel piccolo intestino sono emulsionati dagli acidi biliari e idrolizzati dalla lipasi pancreatica. Questa idrolisi rilascia l'acido grasso della catena principale dell'etanolo portando alla liberazione degli FFA e di una molecola di etanolo. Analogamente ai TG, gli FFA prodotti dagli EE sono assorbiti dagli enterociti dove vengono convertiti in TG e successivamente trasportati nel sangue.

Il metabolismo degli EE è meno efficiente di quello dei TG. La lipasi pancreatica infatti idrolizza gli EE in misura minore e ad un ritmo più lento rispetto TG. ${ }^{10-12}$ L'idrolisi di EPA e DHA può inoltre essere ulteriormente compromessa nei soggetti con disturbi digestivi e con insufficienza pancreatica.

EPA e DHA sono incorporati nei fosfolipidi delle membrane cellulari, in particolare nel cuore e nel cervello, ed immagazzinati nel tessuto adiposo come trigliceridi.

\section{Livelli plasmatici tissutali}

Come per i farmaci di sintesi è necessario chiarire le correlazioni tra dosi di omega-3 assunte e livelli plasmatici/cellulari e tra livelli plasmatici ed effetti biologici. Al momento non è chiaro se esista un valore ottimale di livelli plasmatici al di sotto del quale vi sia un rischio di insorgenza di patologie cardiovascolari ed al di sopra del quale non vi siano ulteriori vantaggi $o$ addirittura insorgenza effetti collaterali.

È stato recentemente proposto l'uso dell'omega-3 index, cioè la valutazione dei livelli di omega-3 a lunga catena nei globuli rossi. L'omega-3 index è stato anche correlato con il rischio cardiovascolare ed è stato dimostrato che basse concentrazioni eritrocitarie di omega-3 rappresentano un fattore indipendente di rischio cardiovascolare.

Certamente vi è un rapporto lineare tra assunzione di EPA e DHA e livelli plasmatici. Ad esempio, dopo somministrazione a vari gruppi di soggetti di EPA e DHA sotto forma di EE incapsulati per un periodo di 6 settimane, si osservano incrementi rispetto ai livelli iniziali correlati in modo perfettamente lineare con le dosi stesse. I PUFA n-3 tendono ad accumularsi a livello di lipidi plasmatici (soprattutto fosfolipidi) e cellulari per lungo tempo. È stata anche dimostrata la protratta permanenza dei PUFA a lunga catena nei lipidi circolanti dopo lunga interruzione (6 settimane) del trattamento. Analogamente EPA e soprattutto DHA, permangono a lungo anche a livello dei lipidi piastrinici, sempre dopo interruzione del trattamento.

\section{Omega-3 in commercio}

Attualmente sono disponibili diversi preparati in commercio a base di omega-3, alcuni classificati come integratori, altri come farmaci. La più grande differenza tra un farmaco e un integratore è che al primo sono attribuiti determinati effetti terapeutici derivati da studi clinici orientati a valutare l'efficacia e la sicurezza mentre è vietato per legge attribuire qualsiasi 
proprietà farmacologica all'integratore. Altra importante differenza tra le due classi riguarda l'obbligo per il farmaco di dimostrare che la composizione in concentrazione e purezza sia standardizzata e ripetibile, obbligo non previsto per gli integratori.

Diversi studi hanno dimostrato che gli omega-3 costituiscono una terapia efficace nella prevenzione del rischio cardiovascolare e della mortalità nel postinfarto e riducono i livelli di trigliceridi nel sangue. Queste proprietà farmacologiche sono però attribuite a concentrazioni di omega-3 di almeno $85 \%$.

I prodotti disponibili in commercio contengono un quantitativo di EPA e DHA dichiarato in etichetta (Santerre C, personal communication, 2007) le cui quantità sono variabili tra $20 \%$ e $95 \%$ e la restante parte è costituita comunemente da altri PUFA n-3 come grassi monoinsaturi, grassi saturi, gelatina o glicerina. ${ }^{13}$ Una capsula da $1 \mathrm{~g}$ quindi può contenere tra 200 e 950 mg di EPA e DHA.

\section{Prescrizione dei farmaci a base di omega-3}

I farmaci PUFA n-3 sono ammessi al rimborso da parte del Servizio Sanitario Nazionale (SSN) per le indicazioni previste dalle Note 13 e 94 dell'Agenzia Italiana del Farmaco (AIFA), ovvero per pazienti con età superiore ai 65 anni, affetti da dislipidemie familiari (iperlipidemia familiare combinata, iperchilomicronemie e gravi ipertrigliceridemie) e iperlipidemie in pazienti con insufficienza renale cronica moderata e grave per livelli di trigliceridi $\geq 500 \mathrm{mg} / \mathrm{dL}$. La nota 94 prevede il rimborso SSN per i pazienti che hanno sofferto una recente sindrome coronarica acuta nella prevenzione della morte improvvisa dopo infarto del miocardio.

\section{Tossicità ed effetti avversi}

Negli Stati Uniti il dipartimento per la salute e lo Human Services Agency for Healthcare Research and Quality hanno identificato 148 studi effettuati sugli omega-3 che riportano eventi avversi in più di 20.000 soggetti. Complicazioni gastrointestinali si sono riscontrate nel $6,6 \%$ dei soggetti trattati con omega-3 rispetto al 4,3\% riscontrati nel gruppo placebo. Non sono stati riportati casi di aumento dell'incidenza di sanguinamento, né casi di decesso o di malattie potenzialmente letali associate all'assunzione di omega-3. Settantasette studi non hanno riportato alcun effetto collaterale. Sulla base di ciò le agenzie hanno concluso che gli eventi avversi associati all'assunzione di olio di pesce o supplementazioni a base di ALA sono bassi.

Inoltre la Food and Drug Administration (FDA) ha dichiarato sicuro il trattamento con EPA + DHA a dosi superiori a $3 \mathrm{~g} / \mathrm{die}$, anche se i dati sono limitati ad un periodo inferiore a 6 mesi.
Uno dei problemi maggiormente discusso in tema di sicurezza è la possibile contaminazione di mercurio conseguente al consumo di pesce e olio di pesce anche se non sono mai stati riportati casi di avvelenamento da mercurio causato dal consumo di pesce negli Stati Uniti negli ultimi 35 anni. Piccoli disturbi neurologici causati dall'esposizione cronica a basse dosi di mercurio nel pesce sono tutt'oggi controverse. L'FDA comunque raccomanda di limitare il consumo di pesce ad alto contenuto di mercurio, nonostante una grossa meta-analisi effettuata da Mozaffarian e Rimm mostra come il rapporto rischio-beneficio sia di 1:400. Inoltre il mercurio è solubile in acqua e si lega alle proteine, quindi si ritrova nelle fibre muscolari del pesce. Per questi motivi i supplementi a base di olio di pesce contengono solo tracce trascurabili di mercurio.

In ultimo, considerando che il cervello allo stadio fetale è maggiormente suscettibile al danno da mercurio rispetto ad un cervello adulto, l'FDA raccomanda di evitare il consumo di pesce ad alto contenuto di mercurio in gravidanza e durante l'allattamento. È stata presa in considerazione anche la possibile correlazione tra somministrazione di omega-3 e insorgenza di cancro alla prostata. Una meta-analisi condotta su 4 coorti e 5 case report concludono che un'alta somministrazione di ALA è associata ad un aumento del rischio di insorgenza di cancro alla prostata ma lo studio è eterogeneo e con significatività statistica solo in 3 casi su 5. Ad oggi sono state riportate associazioni nulle e inverse tra somministrazione di EPA + DHA e aumento del rischio di insorgenza di cancro alla prostata.

\section{Conclusioni}

Di tutti gli studi presenti in letteratura, solo un piccolo numero mostra un assorbimento simile tra TG e oli EE. ${ }^{12,14}$ La maggior parte delle ricerche riscontra un incremento dell'assorbimento e della biodisponibilità di EPA e DHA per omega-3 sottoforma di TG rispetto a $\mathrm{EE}$.

In conclusione è possibile ammettere un vantaggio nell'assunzione di olio di pesce nella forma TG rispetto EE in quanto maggiore è l'assorbimento, la resistenza all'ossidazione e migliore la digeribilità.

\section{Bibliografia}

1. Rubio-Rodríguez N, Beltrán S, Jaime I, et al. Production of omega-3 polyunsaturated fatty acid concentrates: a review. Innov Food Sci Emerg Technol 2010;11:1-12.

2. Schuchardt JP, Neubronner J, Kressel G, et al. Moderate doses of EPA and DHA from re-esterified triacylglycerols but not from ethyl-esters lower fasting serum triacylglycerols in statin-treated dyslipidemic subjects: results from a six month randomized controlled trial. Prostaglandins Leukot Essent Fatty Acids 2011;85:381-6. 
3. Neubronner J, Schuchardt JP, Kressel G, et al. Enhanced increase of omega-3 index in response to long-term n-3 fatty acid supplementation from triacylglycerides versus ethyl esters. Eur J Clin Nutr 2011;65:247-54.

4. Dyerberg J, Madsen P, Møller JM, et al. Bioavailability of marine n-3 fatty acid formulations. Prostaglandins Leukot Essent Fatty Acids 2010;83:137-41.

5. Lawson LD, Hughes BG. Absorption of eicosapentaenoic acid and docosahexaenoic acid from fish oil triacylglycerols or fish oil ethyl esters co-ingested with a high-fat meal. Biochem Biophys Res Commun 1988; 156:960-3.

6. Marsen TA, Pollok M, Oette K, Baldamus CA. Pharmacokinetics of omega-3-fatty acids during ingestion of fish oil preparations. Prostaglandins Leukot Essent Fatty Acids 1992;46:191-6.

7. Lowe ME. Pancreatic triglyceride lipase and colipase: insights into dietary fat digestion. Gastroenterology 1994;107:1524-36.
8. Favé G, Coste TC, Armand M. Physiochemical properties if lipids: new strategies to manage fatty acid bioavailability. Cell Moll Biol (Noisy-le-grand) 2004;50:815-31.

9. Carlier H, Bernard A, Caselli C. Digestion and absorption of polyunsaturated fatty acids. Reprod Nutr Dev 1991;31:475-500.

10. Lawson LD, Hughes BG. Human absorption of fish oil fatty acids as triacylglycerols, free acids, or ethyl esters. Biochem Biophys Res Commun 1988;152: 328-35.

11. el Boustani S, Colette C, Monnier L, et al. Enteral absorption in man of eicosapentaenoic acid in different chemical forms. Lipids 1987;22:711-4.

12. Krokan HE, Bjerve KS, Mork E. The enteral bioavailability of eicosapentaenoic acid and docosahexaenoic acid is as good from ethyl esters as from glyceryl esters in spite of lower hydrolytic rates by pancreatic lipase in vitro. Biochim Biophys Acta 1993;1168:59-67. 


\title{
II ruolo degli omega-3 nel paziente pluripatologico complesso: dalle evidenze alla pratica clinica in Medicina Interna
}

\author{
Andrea Fontanella \\ Dipartimento di Medicina, Ospedale del Buon Consiglio Fatebenefratelli, Napoli, Italia
}

Le caratteristiche degli acidi grassi, le problematiche cliniche a essi connessi e il ruolo degli omega-3 nel paziente pluripatologico complesso sono stati ampiamente ed eccellentemente espressi in questo numero monotematico dei Quaderni.

Nelle conclusioni che mi sono state affidate, oltre a sottolineare alcuni punti focali, preferisco fare delle considerazioni su aspetti contraddittori del problema e alcune valutazioni che rientrano nell'ambito della Wise Medicine.

La recente letteratura scientifica riporta ormai oltre 27.000 studi che dimostrano l'importanza degli omega-3 per l'equilibrio clinico dell'individuo, infatti essi: mantengono in buona salute l'apparato circolatorio e il sistema cardiovascolare, favoriscono un buon benessere psicofisico, preservano una buona funzionalità delle articolazioni, migliorano la memoria, la concentrazione, l'apprendimento e l'umore, mantengono la pelle sana e vitale, contrastandone l'invecchiamento, migliorano lo sviluppo del bambino e la salute della mamma durante la gravidanza, rinforzano il sistema immunitario, riducono il rischio di degenerazione maculare oculare, bilanciano le funzioni del sistema ormonale in menopausa, migliorano il metabolismo dei grassi, prevenendo l'obesità.

L'11 agosto 2015, inoltre, su Nature Communications, ${ }^{1}$ è stato pubblicato on line uno studio che mostra come adolescenti e giovani adulti, considerati ad alto rischio per le psicosi, presentino, rispetto al gruppo placebo, significative riduzioni nella progressione verso il disturbo psicotico a sette anni, dopo un breve

Corrispondente: Andrea Fontanella, Dipartimento di Medicina Ospedale del Buon Consiglio Fatebenefratelli, via Manzoni 220, 80123 Napoli, Italia.

Tel.: +39.335.653.4737.

E-mail: andreafontanella52@gmail.com

Articolo pubblicato secondo la Creative Commons Attribution NonCommercial 3.0 License (CC BY-NC 3.0).

(C) Copyright A. Fontanella, 2015

Licensee PAGEPress, Italy

QUADERNI - Italian Journal of Medicine 2015; 3:306-308 periodo di somministrazione, dodici settimane, di acidi grassi polinsaturi omega-3.

A fronte di tali inequivocabili evidenze e prima di trarre alcune conclusioni sugli aspetti terapeutici e di prevenzione secondaria, che, generalmente, lasciano molti meno dubbi sui vantaggi degli interventi, sembra necessario fare alcune considerazioni proprio sui pazienti fragili e complessi, anziani, cui la monografia si rivolge.

Nel numero di agosto 2015 dell'American Journal of Medicine, ${ }^{2}$ è apparso un articolo che sottolinea come nella prevenzione primaria dell'anziano sia necessario seguire criteri clinici e non prescrivere automaticamente le statine. Questo rafforza quanto già evidenziato dalla dottoressa Pasquini nel suo articolo, che sottolinea come particolare attenzione debba essere rivolta alla categoria d'individui a basso rischio cardiovascolare con livelli non ottimali di colesterolo, dove il trattamento con statine costituisce a oggi materia d'intenso dibattito. L'articolo ${ }^{2}$ parte da un recente editoriale di commento alle ultime linee guida sul trattamento dell'ipercolesterolemia, ${ }^{3}$ nel quale Grundy suggerisce che esse inducono a ipertrattare, in prevenzione primaria, i soggetti nella fascia di età compresa tra 65 e 75 anni, basandosi solo sui numeri dati dalle carte di rischio aterosclerotico per le malattie cardiovascolari. Tale commento viene criticato, affermando che esso è fuorviante perché non riconosce che le linee guida non raccomandano in modo esplicito la prescrizione di statine automaticamente per la prevenzione primaria nei soggetti a basso rischio, compresi nella fascia di età 65-75 anni, bensì specificamente raccomandano una discussione medico - paziente e incoraggiano l'individualizzazione della terapia di riduzione del rischio (Tabella 1 ). ${ }^{2}$

Pertanto la decisione terapeutica deve essere presa secondo il giudizio del clinico, tenendo conto dello stato generale del soggetto e della preferenza del paziente, anche in considerazione del fatto che la revisione delle linee guida eseguita esclusivamente sui trial di prevenzione primaria non ha mostrato alcun beneficio netto nè significativa riduzione dell'infarto e dell'ictus con la terapia con statine in individui a basso rischio, di età compresa tra 40-75 anni.

Alla stessa maniera le linee guida non raccoman- 
dano la terapia con statine automaticamente per la prevenzione primaria negli individui di età superiore a 75 anni, perché la prescrizione delle statine in questi individui richiede considerazione dei fattori aggiuntivi, come le comorbidità, le politerapie, la sicurezza, e le priorità di cura.

Le linee guida attribuiscono all'intervento sugli stili di vita un ruolo chiave nel trattamento dei pazienti con ipercolesterolemia. Dal punto di vista dietetico l'obiettivo è rivolto alla riduzione dell'introito di cibi con effetti negativi sul profilo lipidico; i grassi non dovrebbero superare il 35\% del totale delle calorie introdotte, riducendo i grassi saturi a meno del $6 \%$ e non superando l'introito giornaliero di $200 \mathrm{mg}$ di colesterolo.

Anche su quest'aspetto, mi piace citare un articolo pubblicato on line il 12 agosto su $B M J,{ }^{4}$ in cui, secondo una revisione sistematica e una meta-analisi di studi osservazionali, su individui adulti in apparente buona salute, si rileva come il consumo di grassi saturi non è associato con la mortalità per tutte le cause, con le malattie cardiovascolari, le malattie coronariche (CHD), l'ictus ischemico o il diabete. Al contrario, invece, il consumo di acidi grassi trans è associato con la mortalità per tutte le cause, CHD totali e mortalità per CHD. Tuttavia, gli autori avvertono che i risultati hanno dei bias dipendenti dall'eterogeneità delle evidenze e da limiti metodologici. Quando hanno sintetizzato i risultati degli studi, non hanno trovato alcuna associazione tra assunzione di grassi saturi e mortalità per tutte le cause, nonostante l'inserimento dei dati positivi dal Seven Countries'Study, rilevando, tuttavia, che non era possibile coniugare in modo efficace l'associazione positiva trovata nel Seven Countries 'Study con le associazioni di altri studi, a causa delle differenze metodologiche. Comunque, anche se i grassi saturi non sono stati associati con la malattia coronarica totale, c'è stato un trend di associazione tra grassi saturi e mortalità per CHD.

I ricercatori hanno, invece, etichettato con certezza, come moderata, l'associazione del consumo di grassi trans con gli outcome delle CHD. Si sono, infatti, trovate associazioni positive affidabili e forti tra assunzione di grassi trans e CHD e la mortalità per CHD. Al contrario, l'associazione tra i grassi trans e l'ictus ischemico era meno chiara, e i ricercatori hanno scoperto che i due studi prospettici sul tema hanno prodotto risultati inconsistenti.

Anche se non c'era alcuna associazione tra i grassi trans e il diabete di tipo 2, i dati suggeriscono che 1'acido trans-palmitoleico (trovato nella componente grassa del latte) può essere in grado di proteggere contro il diabete di tipo 2 . Tale acido grasso non è però contenuto negli acidi grassi trans di produzione industriale.

Sempre in tema di diabete, un recente studio italiano, del gruppo di Francesco Violi, ${ }^{5}$ ha dimostrato come, rispetto ad altri tipi di grasso, l'olio extra vergine d'oliva può avere effetti salutari sui livelli di zucchero nel sangue e del colesterolo post-prandiali. Questa può essere una spiegazione in più del perché una dieta mediterranea tradizionale, ricca di olio d'oliva, è legata a un minor rischio di malattie cardiovascolari. Infatti l'abbassamento post-prandiale della glicemia e del colesterolo può essere utile per ridurre gli effetti negativi di glucosio e colesterolo sul sistema cardiovascolare.

Violi e i suoi colleghi hanno testato l'effetto di aggiunta di olio extra vergine di oliva (OEVO) a una dieta mediterranea a base di frutta, verdura, cereali e pesce, con un consumo limitato di latte o carne rossa.

Gli esami ematochimici, eseguiti prima e due ore dopo i pasti, hanno rilevato che la glicemia post-prandiale naturalmente aumenta in tutti, ma molto meno dopo un pasto con olio d'oliva rispetto a uno con olio di mais.

Questo è in linea con le precedenti ricerche che collega OEVO a livelli elevati d'insulina. Non è chiaro, però, perché le analisi del sangue dopo i pasti con olio d'oliva hanno anche mostrato livelli più bassi di lipoproteine a bassa densità. Rispetto al gruppo che assumeva olio di mais.

Si deve comunque tener presente che questo studio sperimentale, fatto su piccoli numeri, non deve essere assunto come il permesso di versare l'OEVO in aggiunta ad ogni pasto, ma che è preferibile usare l'olio

Tabella 1. Elementi di discussione tra medico-paziente per l'avvio della terapia con statina in prevenzione primaria per ogni livello di rischio per patologia aterosclerotica cardiovascolare.

Discutere con ogni paziente (eccetto quelli con LDL colesterolo $\geq 190 \mathrm{mg} / \mathrm{dL}$ o per quelli affetti da diabete mellito di età compresa tra $40-75$ anni e con LDL colesterolo $\geq 70 \mathrm{mg} / \mathrm{dL}$ ):

- Potenziali benefici della terapia con statina nella riduzione del rischio di patologia aterosclerotica cardiovascolare (ASCVD)

- Potenziali eventi avversi e interazioni farmacologiche della terapia con statina

- Adozione di uno stile di vita sano

- Gestione di altri fattori di rischio

- Preferenza del paziente

- Se la decisione é incerta considerare per l'avvio della terapia con statina valori di LDL colesterolo $\geq 160 \mathrm{mg} / \mathrm{dL}$, storia familiare di malattia cardiovascolare prematura, errato stile di vita, calcium score delle arterie coronariche $\geq 300$ Agatson unitá o età $\geq 75$ anni, sesso, razza, proteina C reattiva $\geq 2 \mathrm{mg} / \mathrm{dL}$ o $\mathrm{ABI}<0,9$

ASCVD, rischio aterosclerotico per le malattie cardiovascolari 
extra vergine di oliva al posto di altri grassi; esso deve essere incluso come una fonte di energia nel contesto di una dieta equilibrata.

Tutte queste evidenze, comunque, rendono ragione del fatto che una corretta alimentazione rappresenta la base per la correzione dei fattori di rischio. Molti sono i nutrienti, contenuti nel pesce, frutta, verdura e nocciole, che hanno un effetto positivo sul metabolismo del colesterolo e dei trigliceridi. Sarebbe quindi necessario avere un'alimentazione molto ricca di pesce, possibilmente di taglia piccola in modo da evitare l'inquinamento da mercurio e altre tossine, presenti nei pesci medio-grandi. L'assunzione di questi nutrienti può essere incrementata, ma per raggiungere un effetto terapeutico può essere utile supplementare la dieta con cibi arricchiti o con nutraceutici. ${ }^{6}$

Il fabbisogno minimo quotidiano di omega-3 (come consigliato anche dalla FAO) è l' $1,5 \%$ del fabbisogno calorico giornaliero. Assumendo con l'alimentazione $1800 \mathrm{kcal}$ al giorno si dovrebbero ingerire almeno $3 \mathrm{~g}$ di omega- 3 , dose che in realtà spesso non si riesce a raggiungere, perché gli alimenti ricchi di questi grassi non sono molto appetibili, e non tutti vogliono o riescono a mangiare ogni giorno pesce. Diventa quindi necessaria la scelta di un'integrazione, specie se vi sono condizioni patologiche che lo richiedano.

$\mathrm{Al}$ termine di queste mie conclusioni ritengo necessarie alcune considerazioni di ordine pratico, specialmente in quest'epoca di medicina fai da te o medicina da supermercato.

Per mantenersi in salute e prevenire molte malattie basta mangiare molto pesce oppure prendere ogni giorno una capsula o più di omega-3, magari acquistata al supermercato. Peccato che non sia così: il $90 \%$ dei prodotti venduti da supermercati, erboristerie, negozi online e perfino farmacie sono quanto meno inefficaci, se non dannosi per la salute.

Vi sono confezioni anche di marche molto pubblicizzate e vendute in farmacia nel cui interno ci sono $17 \mathrm{~g}$ di omega-3 (indicati anche come l'acido eicosapentaenoico o EPA e l'acido docosaesaenoico o DHA) per 100 grammi di prodotto, ovvero solo il $17 \%$. Talune confezioni in vendita nei supermercati ne contengono solo il $12 \%$. È ben noto da numerosi studi, che queste sostanze costituiscono un'efficace terapia preventiva del rischio cardiovascolare e della mortalità nel post-infarto, oltre ad essere in grado di ridurre i livelli di trigliceridi nel sangue, ma solo quando assunti a una concentrazione di almeno $1^{\prime} 85 \%$. Vi è ancora un altro aspetto molto importante: se l'olio di pesce è di bassa qualità, a causa dell'inquinamento marino è possibile trovare nel suo interno degli inquinanti molto pericolosi come il mercurio, $\mathrm{o}$ addirittura cancerogeni come le diossine e il policlorobifenile (PCB). ${ }^{7}$

Naturalmente esistono dei prodotti farmaceutici di altissima qualità: i prodotti farmaceutici certificati o integratori di olio di pesce che contengono una percentuale molto elevata di omega-3 (superiore al 60\%) e che sottopongono ogni lotto di produzione all'analisi di un istituto indipendente, riconosciuto a livello mondiale dai principali produttori di omega-3. Tali prodotti, di qualità farmacologica, utilizzano olio praticamente puro al $100 \%$, che, pertanto è chiamato ultrapurificato.

Nell'olio di pesce ultrapurificato le sostanze nocive, come i PCB o il diclorodifeniltricloroetano, ${ }^{7}$ sono ridotte a livelli bassissimi, grazie ad un processo di raffinazione che esclude anche l'eventualità di essere colpiti da disturbi gastrointestinali spesso associati a oli di pesce di qualità inferiore.

Un olio di pesce meno purificato provocherebbe un accumulo d'impurità tossiche che si sono dimostrate capaci di danneggiare il sistema ormonale, causando fastidiosi disturbi intestinali (formazione di gas, dolori addominali e diarrea), ed essere potenzialmente cancerogene.

D'accordo, quindi, sui nutraceutici ma che siano di qualità, garantiti e certificati.

\section{Bibliografia}

1. Amminger GP, Schäfer MR, Schlögelhofer M, et al. Longer-term outcome in the prevention of psychotic disorders by the Vienna omega-3 study. BMJ 2015 [Epub ahead of print].

2. Stone NJ, Blum CB. Primary risk prevention in the elderly: use clinician-patient discussions, not automatic statin prescribing. Am J Med 2015;128:804-6.

3. Scott M. Grundy. Cholesterol management in older persons. Am J Med 2014;127:1140-1.

4. de Souza RJ, Mente A, Maroleanu A, et al. Intake of saturated and trans unsaturated fatty acids and risk of all cause mortality, cardiovascular disease, and type 2 diabetes: systematic review and meta-analysis of observational studies. BMJ 2015;351:h3978.

5. Violi F, Loffredo L, Pignatelli P, et al. Extra virgin olive oil use is associated with improved post-prandial blood glucose and LDL cholesterol in healthy subjects. Nutr Diabet 2015;5:e172.

6. Mannarino M, Ministrini S, Pirro M. Nutraceuticals for the treatment of hypercholesterolemia. Eur J Intern Med 2014;25:592-9.

7. Istituto Superiore di Sanità. Schede chimico-tossicologiche (sostanze organiche). Allegato 5. Disponibile su: www.iss.it/binary/fisr/cont/schede_chim.tossicologiche.pdf 


\section{LINEE GUIDA PER GLI AUTORI}

I Quaderni dell'Italian Journal of Medicine (Quaderni ITJM), costituiscono una collana supplementare solo online annessa alla rivista Italian Journal of Medicine contenente lavori solo in lingua italiana.

I Quaderni ITJM pubblicano:

- Monografie ad hoc individuate dal Presidente FADOI, dal Consiglio Direttivo, dal Board Scientifico o dall'Editor in Chief dell'Italian Journal of Medicine, in funzione del contesto scientifico-istituzionale attuale.

- Monografie ad hoc su temi di particolare rilevanza scientifica a cura della Commissione FADOI Giovani, coordinata dalla Dr.ssa Paola Gnerre.

- Traduzioni in italiano di alcuni lavori pubblicati sui numeri standard dell'Italian Journal of Medicine, di particolare interesse per la comunità scientifica. La selezione è a cura dell'Editor-inChief, Prof. Roberto Nardi.

\section{STESURA DEI LAVORI}

I lavori dovranno essere redatti in modo conforme alle linee guida sotto riportate:

- I manoscritti devono essere scritti interamente in lingua italiana, su documento di Word, con carattere Times New Roman/Arial, dimensione 12, formato A4, interlinea doppia e margini $2,54 \mathrm{~cm}$. Parole totali: $\max 4000 ;$ Sommario/Abstract: $\max 250$ parole; Bibliografia: $\min 40$ voci; Tabelle e Figure: $3 / 5$ totali (le tabelle non devono superare $\mathrm{n} .1$ pagina del documento in Word).

- La strutturazione del contenuto deve attenersi agli standard internazionali per la Rassegna (Review): i) Abstract riassuntivo dell'intero lavoro; ii) Introduzione al tema trattato; iii) Criteri e strumenti di ricerca (criteri di inclusione/esclusione, banche dati consultate, ...); iv) i successivi paragrafi devono illustrare le più recenti scoperte scientifiche nel settore; v) Conclusioni; vi) Bibliografia.

- La prima pagina deve riportare: i) titolo (in stampatello minuscolo), senza acronimi; ii) nome e cognome per esteso di ciascun autore; iii) affiliazione(i) di ciascun autore, numerate con numeri arabi; iv) eventuali ringraziamenti; v) nome e indirizzo postale completi dell'autore corrispondente, corredati da telefono, fax, e-mail; vi) da 3 a 5 parole chiave, separate da virgola. La seconda pagina può riportare: i) contributi degli autori, e.g. informazioni relative a contributi sostanziali delle persone coinvolte nello studio (http://www.icmje.org/\#author); ii) dichiarazione relativa a potenziali conflitti d'interesse; iii) ulteriori informazioni (e.g. fondi, esposizioni durante conferenze...).

- In caso di utilizzo di tabelle, queste devono essere tutte numerate con numeri arabi e citate nel testo in ordine consecutivo (e.g. NON nominare le tabelle come Tabella $1 \mathrm{~A}, 1 \mathrm{~B}, \ldots$ o $1.0,1.1, \ldots)$. Le tabelle devono essere presentate in formato editabile. Ciascuna tabella deve essere corredata da una breve didascalia; in caso di abbreviazioni, riportare una nota a piè di CIASCUNA tabella che spieghi TUTTE le abbreviazioni presenti in ognuna.

- In caso di utilizzo di figure, queste devono essere inviate in formato .tiff o .jpg, allegate al manoscritto in singoli files, secondo le seguenti specifiche:

i) a colori (salvate in modalità CMYK): minimo 300 dpi di risoluzione;

ii) in bianco e nero: minimo 600 dpi di risoluzione;

iii) minimo $17,5 \mathrm{~cm}$ di larghezza.

Ciascuna figura deve essere corredata da una breve didascalia.

$N B$ : In caso di Tabelle/Figure riprese e/o modificate da altri lavori già pubblicati, sarà cura degli autori accertarsi se tali materiali siano o meno coperti da copyright e procurarsi i permessi necessari per la riproduzione. Tali permessi dovranno essere allegati alla versione definitiva del lavoro. L'ufficio editoriale si riserva la facoltà di rimuovere Tabelle/Figure coperte da copyright, se sprovviste dei necessari permessi.

- In caso di utilizzo di abbreviazioni, la prima volta che esse sono citate è necessario scrivere per esteso la definizione+abbreviazione tra parentesi tonde [e.g. risonanza magnetica $(\mathrm{RMN})]$, a seguire si dovrà riportare solo l'abbreviazione (unica eccezione: nei titoli e nelle didascalie di tabelle e figure NON si utilizzano abbreviazioni).

\section{BIBLIOGRAFIA}

Le voci bibliografiche devono essere formattate secondo lo stile Vancouver.

Nella sezione Bibliografia, le voci bibliografiche devono essere numerate consecutivamente nell'ordine in cui appaiono per la prima volta nel testo (NON in ordine alfabetico) e, nel testo, devono essere indicate con numeri arabi in apice. Voci bibliografiche riferite a comunicazioni personali o dati non pubblicati devono essere incorporate nel testo e NON inserite tra le voci numerate [e.g. (Wright 2011, dati non pubblicati) o (Wright 2011, comunicazione personale)]. Le voci bibliografiche nella sezione Bibliografia devono tassativamente essere preparate come segue:

i) più di 3 autori, citare 3 autori, et al. Se il lavoro contiene solo 4 autori, citarli tutti e 4 ;

ii) titolo del lavoro in stampatello minuscolo;

iii) nome della rivista, senza punti, abbreviato secondo gli standard internazionali; in caso di dubbi sulla corretta abbreviazione, fare riferimento ai seguenti siti:

a. ISI Journal Abbreviations Index (http://library.caltech.edu/ reference/abbreviations/);

b. Biological Journals and Abbreviations (http://home.ncifcrf. gov/research/bja/);

c. Medline List of Journal Titles (ftp://ftp.ncbi.nih.gov/pubmed/J_Medline.txt);

iv) inserire l'anno di pubblicazione subito dopo il nome della rivista, seguito da punto e virgola;

v) NON inserire giorno o mese di pubblicazione;

vi) citare solo il volume, seguito dai due punti (NON citare il fascicolo tra parentesi);

vii) abbreviare le pagine, e.g. 351-8

Per accertarsi di aver correttamente formattato le voci bibliografiche, confrontarle con le citazioni in PubMed (http://www.ncbi. nlm.nih.gov/pubmed).

Esempi (prestare attenzione anche alla punteggiatura):

Articolo standard su Rivista

Halpern SD, Ubel PA, Caplan AL. Solid-organ transplantation in HIV-infected patients. N Engl J Med 2002;347:284-7.

Proceedings

Christensen S, Oppacher F. An analysis of Koza's computational effort statistic for genetic programming. In: Foster JA, Lutton E, Miller J, Ryan C, Tettamanzi AG, eds. Genetic programming. EuroGP 2002: Proceedings of the 5th European Conference on Genetic Programming, 2002 Apr 3-5, Kinsdale, Ireland. Berlin: Springer; 2002. pp 182-91.

Articoli i cui autori sono Organizzazioni

Diabetes Prevention Program Research Group. Hypertension, insulin, and proinsulin in participants with impaired glucose tolerance. Hypertension 2002;40:679-86.

Libri

Murray PR, Rosenthal KS, Kobayashi GS, Pfaller MA. Medical microbiology. 4th ed. St. Louis, MO: Mosby; 2002. (CITAZIONE DEL LIBRO INTERO)

Meltzer PS, Kallioniemi A, Trent JM. Chromosome alterations in human solid tumors. In: Vogelstein B, Kinzler KW, eds. The genetic basis of human cancer. New York, NY: McGraw-Hill; 2002. pp 93113. (CITAZIONE DI UN CAPITOLO)

\section{MODALITÀ D'INVIO DEI LAVORI}

Monografie

Gli Autori dovranno fare riferimento all'Editor-in-Chief o alle persone da lui designate nelle lettere di invito a scrivere gli articoli programmati.

Per gli articoli inviati da giovani internisti, gli Autori dovranno fare riferimento alla Dr.ssa Paola Gnerre (e-mail: pgnerre@yahoo.it) e agli eventuali altri referenti da lei designati, nelle prime fasi di stesura dei manoscritti, revisioni e correzioni. 


\section{QUADERNI}

dell'Italian Journal

of Medicine

La Dr.ssa Gnerre raccoglierà poi le versioni definitive dei lavori di ciascuna monografia e provvederà all'invio di tutti i materiali all'ufficio editoriale.

I lavori solo nella loro versione definitiva e approvata dalla Commissione FADOI Giovani dovranno pervenire all'ufficio editoriale già pronti per l'impaginazione e immediata pubblicazione (già corredati da eventuali permessi per la riproduzione di tabelle e immagini).

\section{Traduzioni}

Previo invito dell'Editor-in-Chief, gli Autori dovranno far pervenire all'ufficio editoriale la versione tradotta in italiano, al seguente indirizzo e-mail:paola.granata@pagepress.org

Il file in formato Word dovrà essere formattato secondo gli standard editoriali della rivista ufficiale ed essere già pronto per impagina- zione e immediata pubblicazione (corredato da eventuali permessi per la riproduzione di tabelle e immagini).

Si prega di inviare le eventuali tabelle in formato editabile e le figure in alta definizione secondo gli standard sopra riportati.

\section{NOTA PER GLI AUTORI}

I lavori pubblicati sui Quaderni ITJM non verranno indicizzati, ma saranno liberamente disponibili in un'apposita sezione del sito FADOI (http://www.fadoi.org/).

Gli Autori i cui lavori siano accettati per la pubblicazione sui Quaderni ITJM e che fossero interessati a vederli pubblicati anche sulla rivista ufficiale, dovranno sottomettere attraverso il sito dell'ITJM (www.italjmed.org) la versione (già tradotta) in inglese e redatta in modo conforme alle linee guida della rivista; seguiranno poi la procedura di selezione tramite peer review e, se accettati, saranno inseriti nel piano editoriale standard.

\section{STAFF EDITORIALE}

Paola Granata, Journal Manager paola.granata@pagepress.org

Selvaggia Stefanelli, Marketing Manager marketing@pagepress.org

Claudia Castellano, Production Editor

Tiziano Taccini, Technical Support

\section{QUADERNI - ITALIAN JOURNAL OF MEDICINE}

Tutti gli articoli pubblicati sui QUADERNI - Italian Journal of Medicine sono redatti sotto la responsabilità degli Autori. La pubblicazione o la ristampa degli articoli della rivista deve essere autorizzata per iscritto dall'editore. Ai sensi dell'art. 13 del D.Lgs 196/03, i dati di tutti i lettori saranno trattati sia manualmente, sia con strumenti informatici e saranno utilizzati per l'invio di questa e di altre pubblicazioni e di materiale informativo e promozionale. Le modalità di trattamento saranno conformi a quanto previsto dall'art. 11 del D.Lgs 196/03. I dati potranno essere comunicati a soggetti con i quali PAGEPress intrattiene rapporti contrattuali necessari per l'invio delle copie della rivista. Il titolare del trattamento dei dati è PAGEPress Srl, via Belli 7 - 27100 Pavia, al quale il lettore si potrà rivolgere per chiedere l'aggiornamento, l'integrazione, la cancellazione e ogni altra operazione di cui all'art. 7 del D.Lgs 196/03.

Editore: PAGEPress srl, via G. Belli 7, 27100 Pavia, Italy www.pagepress.org

\section{PUBBLICATO DA}

PAGEPress Publications

via G. Belli 7

27100 Pavia, Italy

T. +39.0382 .1751762$

F: +39.0382 .1750481$

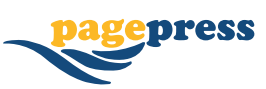

www.pagepress.org

info@pagepress.org 